\title{
Scanning Tunneling Spectroscopy of Rare Earth Hexaborides
}

\author{
Dissertation \\ zur Erlangung des mathematisch-naturwissenschaftlichen Doktorgrades \\ "Doctor rerum naturalium" \\ der Georg-August-Universität Göttingen \\ im Promotionsstudiengang Physik \\ der Georg-August University School of Science (GAUSS)
}

vorgelegt von

Philipp Buchsteiner

aus Hagenow

Göttingen, 2020 
Betreuungsausschuss

PD Dr. Martin Wenderoth

IV. Physikalisches Institut, Georg-August-Universität Göttingen

Prof. Dr. Stefan Mathias

I. Physikalisches Institut, Georg-August-Universität Göttingen

Prof. Dr. Peter Blöchl

Institut für Theoretische Physik, TU Clausthal-Zellerfeld

\section{$\underline{\text { Mitglieder der Prüfungskommission }}$}

Referent: PD Dr. Martin Wenderoth

IV. Physikalisches Institut, Georg-August-Universität Göttingen

Korreferent: Prof. Dr. Stefan Mathias

I. Physikalisches Institut, Georg-August-Universität Göttingen

Weitere Mitglieder der Prüfungskommission:

Prof. Dr. Peter Blöchl

Institut für Theoretische Physik, TU Clausthal-Zellerfeld

Prof. Dr. Hans Hofsäss

II. Physikalisches Institut, Georg-August-Universität Göttingen

Prof. Dr. Michael Seibt

IV. Physikalisches Institut, Georg-August-Universität Göttingen

Prof. Dr. Christian Jooß

Institut für Materialphysik, Georg-August-Universität Göttingen

Tag der mündlichen Prüfung: 25. September 2020 


\section{Contents}

1 Introduction $\quad 3$

2 Rare earth hexaborides $\quad 5$

3 Sample preparation 9

4 Crystal examination

by gamma-ray diffraction 11

5 Scanning tunneling microscopy $\quad 15$

5.1 Theory . . . . . . . . . . . . . . . . . . 16

5.2 Measurement modes . . . . . . . . . . . . . . . . 17

5.2.1 Constant current topography . . . . . . . . . . . 18

5.2 .2 Multi-bias imaging . . . . . . . . . . . . . . 18

5.2.3 Apparent barrier height . . . . . . . . . . . . . . . 19

5.2.4 Scanning tunneling spectroscopy . . . . . . . . . . 20

5.2 .5 Topography normalization . . . . . . . . . . . 21

6 Spatially resolved low-energy electron diffraction 23

7 Surface resonance of the $(2 \times 1)$ reconstructed lanthanum hexaboride (001)-cleavage plane:

A combined STM and DFT study $\quad 25$

7.1 Original publication . . . . . . . . . . . . 27 
8 Surface analysis of the $\operatorname{PrB}_{6}(001)$ cleavage plane by scanning tunneling microscopy and spectroscopy 51

8.1 Original publication . . . . . . . . . . . . . . 53

9 Comparison of the $\mathrm{LaB}_{6}$ and $\operatorname{PrB}_{6}$ results 71

9.1 Chainlike $(2 \times 1)$ reconstruction $\ldots \ldots \ldots 71$

9.2 Uniform terminations . . . . . . . . . . . . . . . 72

9.3 Bulk and surface defects . . . . . . . . . . . 73

9.4 Spectral properties . . . . . . . . . . . . . . . 74

9.5 Corrugation dependance on the bias voltage . . . . . . . . . 75

9.6 Spatially resolved LEED . . . . . . . . . . . . . . . . . 76

$\begin{array}{ll}10 \text { Outlook } & 77\end{array}$

$\begin{array}{ll}\text { Bibliography } & 79\end{array}$

$\begin{array}{lr}\text { Publications, communications and supervised theses } & 84\end{array}$

$\begin{array}{lr}\text { Danksagung } & 87\end{array}$

$\begin{array}{lr}\text { Additional notes } & 89\end{array}$ 


\section{Chapter 1}

\section{Introduction}

The beginning of human civilization was shaped by several factors, such as cultural rites, legal structure and the technology that was utilized. The utter importance of the working materials impressively manifests in the name-giving of several archeological periods themself, i.e. the stone age, the bronze age or the iron age. However, the civilizational progress of the 21 st century is largely shaped by the rapid developments in communication technology [93]. The broad choice of modern materials harbors an immense innovative potential to push the boundaries of today's technologic limitations. Here, a key aspect for the material utilization is the steady device miniaturization. Up to now, commercially available transistors rely on device structures in the nanometer regime [18]. Recently, the ability to read and write the states of a single atom was demonstrated [52]. Although it seems like the final goal in miniaturization is reached, quantum computing could enable larger innovative opportunities [42].

Such a continuous development requires a profound understanding of the scientific background of the materials. Especially the upcoming opportunities regarding quantum computing demand fundamental research. Although the basic concepts of quantum mechanics have been developed over 100 years ago, interacting many-body systems and electronic correlation in condensed matter are an ongoing research field [84, 85]. Electronic correlation is oftentimes addressed with the $4 f$ electrons of rare earth elements. These core-like electrons can have large magnetic moments and the delicate interplay with itinerant conduction elec- 
trons via the RKKY-interaction enables numerous exotic ground states [84]. The emergent phenomena such as high temperature superconductivity or colossal magnetoresistance [77] make the $4 f$ materials of great interest for novel applications.

A well-suited model system for the exploration of $4 f$ electron physics are rare earth hexaborides $\left(R \mathrm{~B}_{6}\right)$. While the crystal structure is fairly simple, varying the rare earth element changes the $4 f$ electron count from $4 f^{0}$ in La to $4 f^{14}$ in $\mathrm{Lu}$ and with that dramatically the electronic properties. For instance, $\mathrm{CeB}_{6}$ is a dense Kondo system with complex magnetic order [98], $\mathrm{YB}_{6}$ a superconductor [39], $\mathrm{EuB}_{6}$ a ferromagnetic semimetal [65] and $\mathrm{SmB}_{6}$ a Kondo insulator [20, 92]. One of the most researched hexaborides is $\mathrm{LaB}_{6}$, which is used for decades as high efficient electron emitter [96]. Although numerous bulk studies on hexaborides have been performed, the surface physics became of growing interest as $\mathrm{SmB}_{6}$ was argued to host topologically protected states [21]. To test this hypothesis transport measurements, angle-resolved photoemission and scanning tunneling microscopy were performed, but an unambiguous evidence of the topological nature of the surface states is still missing [21]. Apparently, understanding hexaboride surface physics is far from trivial and complementary aspects of surface science, materials science and computational physics are required to disentangle the exotic ground state properties.

This thesis is part of a joint approach of experiment and theory in an effort to quantify the $4 f$ physics of hexaboride surfaces. We combined scanning tunneling microscopy and spectroscopy, density functional theory and low-energy electron diffraction on the $\mathrm{LaB}_{6}$ and $\mathrm{PrB}_{6}$ (001)-cleavage planes. The goal of our work is to provide a thorough characterization of the respective surfaces, which is inevitable for their technical utilization.

The thesis is organized as follows: After this introductory part a description of rare earth hexaborides is presented. Then, the main experimental techniques which I used for this thesis are explained, namely scanning tunneling microscopy and spectroscopy, gamma-ray diffraction and low-energy electron diffration. Centerpiece of this manuscript are the results of $\mathrm{LaB}_{6}$ and $\operatorname{PrB}_{6}$, which have been submitted to scientific journals. To provide an increased readability, each paper is separately introduced. Finally, these two studies were summarized, compared to each other and an outlook is given in the final chapter. 


\section{Chapter 2}

\section{Rare earth hexaborides}

In this thesis, the surface properties of two rare earth hexaborides $\left(R \mathrm{~B}_{6}\right)$ were investigated, namely $\mathrm{LaB}_{6}$ and $\mathrm{PrB}_{6}$. But before the specific surface properties of these crystals are elucidated, a thorough overview of the general bulk structure of this material class will be given as it consequently affects the cleavage behavior. Moreover, some facinating properties of the hexaborides are briefly reviewed and the technological relevance is emphasized as well.

a)

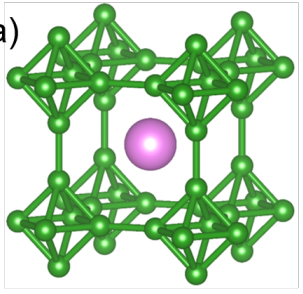

b)

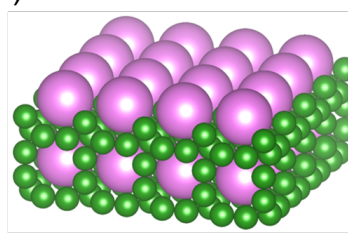

$(001) \quad c)$

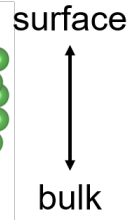

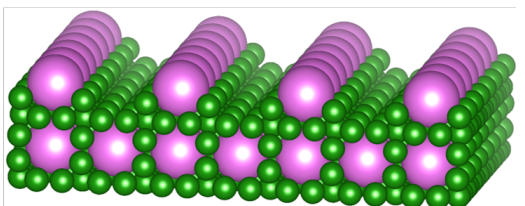

Figure 2.1: a) Simple cubic crystal structure of $R \mathrm{~B}_{6}$. The boron atoms are depicted in green and the rare earth atom in pink. b) Scheme of a uniform rare earth terminated (001) surface. c) Scheme of a chainlike $(2 \times 1)$ terminated (001) surface. The schematic drawings were created with VESTA [51].

The hexaborides share a simple cubic crystal structure with $P m \overline{3} m$ symmetry, as seen in Fig. 2.1 (a). The six boron atoms are arranged in an octahedron and are connected by covalent bonds within and in between the neighboring octahedra [13]. Therefore, the hexaborides are characterized by a three-dimensional covalent binding network, which results generally in high stability and hardness [13]. However, the boron network itself is electron deficient and requires addi- 
tional electron donation. Former calculations showed, that for stabilizing a $\mathrm{B}_{6}$ unit 20 electrons are needed [43]. Boron has only three valence electrons and therefore only 18 electrons can be contributed by the $\mathrm{B}_{6}$ unit itself. Alkaline earth metals such as $\mathrm{Ca}$ or $\mathrm{Sr}$ can share their two valence electrons and hence stabilize the boron framework. In the resulting crystal, these metal ions are situated in the center of the unit cell and hence they are 24-fold coordinated [13]. Furthermore, the electron donation from the metal ion to the boron network creates an ionic character of the crystal. Therefore, when creating the (001) surface, uniform rare earth or $\mathrm{B}_{6}$ terminations are polar and a chainlike $(2 \times 1)$ reconstructions may be present. For a scheme of a uniform rare earth and a chainlike $(2 \times 1)$ reconstructions see Fig. 2.1 b) and c), respectively.

Substituting the divalent with a trivalent metal typically results in metallic conductivity [43]. By introducing rare earth elements to the crystal, versatile correlation effects can arise due to the $4 f$ occupancy. $\mathrm{CeB}_{6}$ for instance is a dense Kondo system [90] with complex magnetic order, including antiferromagnetic and quadrupolar ordering [98]. Most $R \mathrm{~B}_{6}$ order antiferromagnetically at low temperature, such as $\mathrm{PrB}_{6}, \mathrm{NdB}_{6}, \mathrm{GdB}_{6}, \mathrm{TbB}_{6}, \mathrm{DyB}_{6}$ [24], and $\mathrm{HoB}_{6}$ [35]. Eu has a half-filled $4 f$ shell and $\mathrm{EuB}_{6}$ is a ferromagnetic semimetal [65]. One of the most prominent hexaborides is $\mathrm{SmB}_{6}$, which is a Kondo insulator and proposed to host topologically protected states $[20,92]$. In $\mathrm{YbB}_{6}$ topologically protected states in absence of a Kondo mechanism are suggested [53] and superconducting behavior is found in $\mathrm{YB}_{6}$ [39]. As evident from the short list above, the properties of $R \mathrm{~B}_{6}$ are widely tunable while remaining the fairly simple crystal structure.

In this thesis we will focus on the surface physics of hexaborides. The surface physics of a material is drastically altered from the respective bulk compound due to the reduced dimensionality. The surface atoms obviously have fewer neighbors than the atoms in the bulk material, which inevitably modifies the chemical bonds and consequently the electronic structure [44]. Furthermore, the surface atoms can rearrange due to relaxation and reconstruction [44] and each resulting termination can have a unique electronic structure.

The immense interest in hexaboride surfaces started with the discovery of the astonishing low work-function of $\mathrm{LaB}_{6}$. Lafferty pointed out the superiror cathode properties, namely high mechanical stability, a high melting point, stability 
in vacuum and a work function of $2.66 \mathrm{eV}$ [40]. For the elements, a low work function is usually accompanied with high chemical reactivity. For example, the highly reactive Cesium has a work function of $1.9 \mathrm{eV}$ [87]. The chemically stable noble metals Gold and Platinum have work functions of at least two times the work function of Ceasium each [72, 19]. $\mathrm{LaB}_{6}$ combines these two seemingly contradicting properties and consequently found wide usage as highly efficient electron emitter. Apart from the $\mathrm{LaB}_{6}$ surface the $\mathrm{SmB}_{6}$ surface attracted much attention, since it is proposed to be a topological insulator, as mentioned above. Therefore, $\mathrm{SmB}_{6}$ is argued to play a crucial role in future spintronics or quantum computing applications [31].

All these apparent and promising applications require a thorough sample and surface preparation. In the upcoming chapter the crystal growth of our samples is reviewed and the developed surface preparation is displayed in more detail. 


\section{Rare earth hexaborides}




\section{Chapter 3}

\section{Sample preparation}

To investigate the (001)-cleavage planes of $\mathrm{LaB}_{6}$ and $\operatorname{PrB}_{6}$, several preparation steps are required. Firstly, high quality single crystals are needed to be grown. Secondly, the crystals have to be oriented to find the equivalent $\{001\}$-planes on the macroscopic crystal boules. Since the crystal dimensions are of several $\mathrm{cm}$ length, a further processing is necessary to downsize the crystal into cleavable samples. In the following, these steps are displayed in more detail.

Hexaboride single crystals are commonly grown by Al-flux. However, Al-flux grown crystals tend to be too small and too impure [63]. Hence, floating-zone growth is the method of choice. The floating-zone growth was carried out by Dr. Monica Ciomaga Hatnean and Prof. Geetha Balakrishnan at the University of Warwick and further information about the growth process itself can be found in Refs. $[6,7]$. The received single crystals are of cylindrical shape with a thickness of few $\mathrm{mm}$ and a length of few cm, see Fig. $3.1 \mathrm{a}$ ).

The growth axis, however, does not necessarily coincide with the cylinder axis or one of the principle $\{001\}$-crystal planes. To find the principle crystallographic axes, the crystals needed to be oriented. Due to the rather large crystal dimensions the orientation was done by gamma ray diffraction. The small wave length of the used gamma-rays, $0.0392 \AA$ in the present setup, leads to a negligible absorption for the examined samples. Since the crystal structure of $\mathrm{LaB}_{6}$ and $\operatorname{PrB}_{6}$ is already known, the detector is set to the expected Bragg-angle $\theta_{001}$ position of a (001)- 
reflex for the $d_{001}$ plane via

$$
n \lambda=2 d_{001} \sin \left(\theta_{001}\right)
$$

The actual orientation was done by rotating the crystal, which was mounted onto a goniometer, until the respective $\{001\}$ signals appeared. Further results of the gamma-ray diffraction are displayed in Chapter 4. After finding the principle axes, one of these axes is marked onto the sample by making a parallel cut along the (001)-plane at the sample surface, called orientation cut. Perpendicular to the orientation cut the cylindrical crystal is cut into slices of about $300 \mu \mathrm{m}$ thickness, see Fig. 3.1. Each slice is further downsized to rectangular shaped pieces of about $4 \mathrm{~mm} \times 1 \mathrm{~mm}$ size. Since the hexaborides are rather hard and brittle due to the strong covalent binding network, only a diamond coated steel-wire saw was suitable to reproducibly perform the cuts. On top of these rectangular samples a lever is mounted to make the cleavage within the UHV setup more easily. Afterwards the crystals are clamped into the sample holder and transferred into the STM UHV chamber. Here, the samples are cleaved in situ at room temperature and are transferred immediately into the STM head. An exemplary photo of the cleaved sample within the STM head is shown in Fig. 3.1 f).

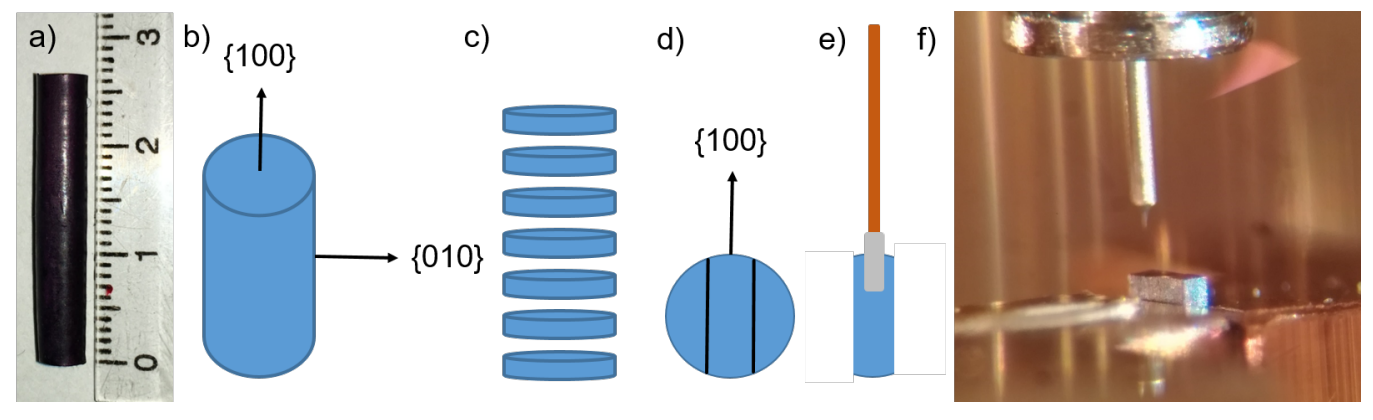

Figure 3.1: a) Photo of a $\operatorname{PrB}_{6}$ single crystal with cm-scale. Photo was sent by Geetha Balakrishnan. b) Scheme of the cylindrical crystal with the indicated crystal planes. c) The crystal is cut into slices of about $300 \mu \mathrm{m}$ thickness. d) These slices are cut again into rectangular samples. e) Finally, a lever is mounted on top and a scratch to initiate the cleavage is added. f) Photo of a cleaved $\mathrm{PrB}_{6}$ sample inside the STM head. The tunneling tip is above the sample. The photo in $\mathrm{f}$ ) was used by Lisa Harmsen within her master thesis as well [30]. 


\section{Chapter 4}

\section{Crystal examination by gamma-ray diffraction}

In the following, the outcome of the gamma-ray diffraction experiments is examined. As described in Chapter 3, the gamma-ray diffraction is necessary for the crystal orientation. Additionally, the crystal quality was examined. We believe, that the found differences in crystal quality between $\mathrm{LaB}_{6}$ and $\operatorname{PrB}_{6}$ could have influenced the resulting surface quality, which will be described in more detail in Chapter 9. As the crystal quality for $\operatorname{PrB}_{6}$ was remarkably higher, we will start the examination with $\operatorname{PrB}_{6}$.

Again, primary goal of the gamma-ray diffraction experiments was the crystal orientation of the (001)-planes for the sample preparation. Therefore, the detector was set to the expected Bragg-angle to find the growth axis. Then, the crystal was rotated by $\omega$ in a wide angular range to find the other equivalent planes. Such a measurement is shown in Fig. 4.1 a). Here, two clear delta-peaks were observed for the (010) and (001) direction, which are about $89.74^{\circ}$ apart. This is in sufficient agreement with the expected $90^{\circ}$ anticipated from the simple cubic crystal structure. Furthermore, the occurance of only two and not more peaks indicate that no additional grains are present and that the crystal is indeed a single crystal.

The single crystal quality can also be checked by a close examination of a $\{100\}$-peak with a finer $\omega$-stepwidth. Figure 4.1 (b) shows such a so-called "rocking-curve". The intensity distribution shows rather a gaussian behavior than 


\section{Crystal examination}

by gamma-ray diffraction
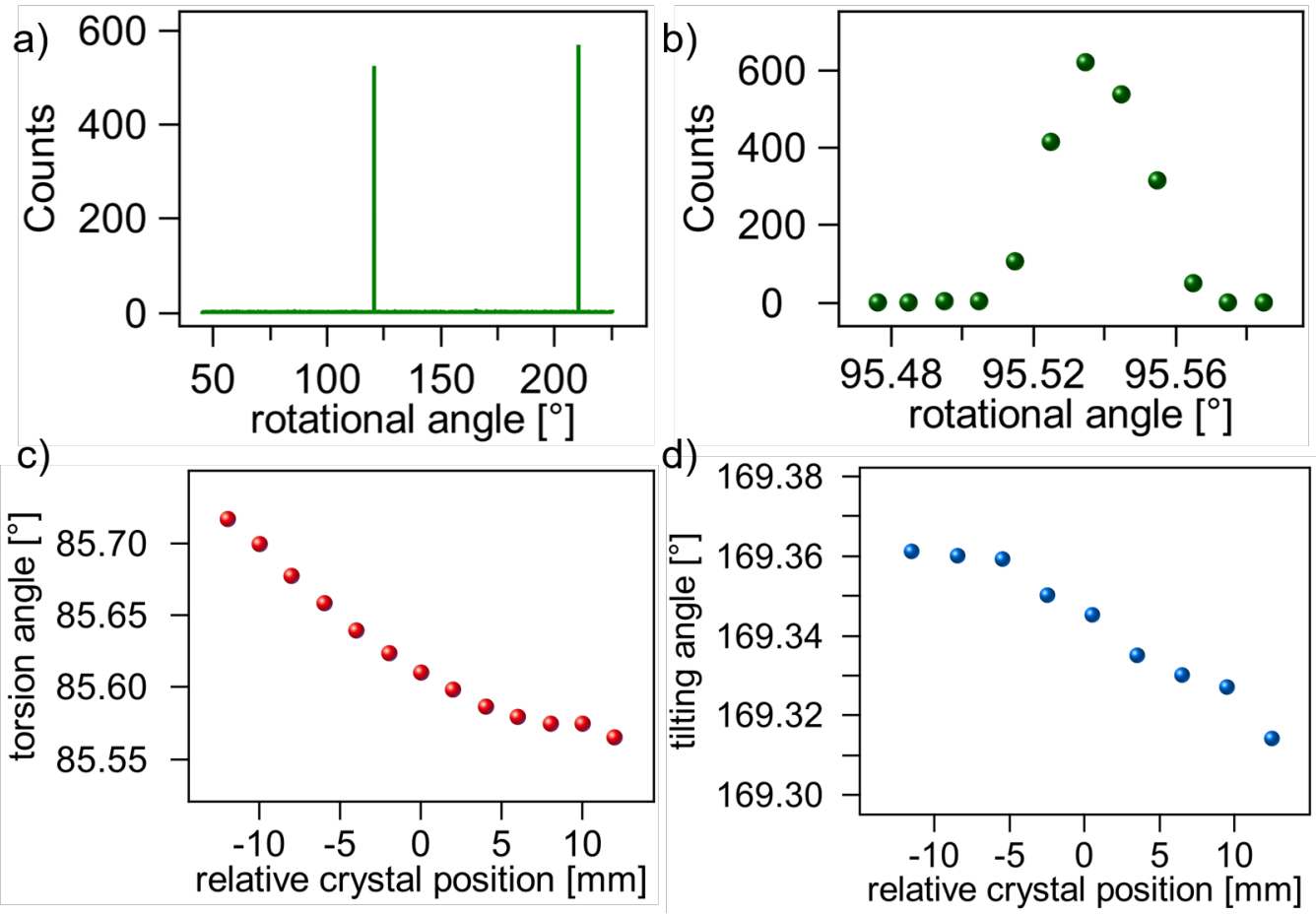

Figure 4.1: a) The crystal was rotated in a wide angular range and two $\{100\}$ peaks were found. b) Rocking-curve of a $\{100\}$-peak. c) Torsion measurement of the $\operatorname{PrB}_{6}$ single crystal. d) Tilt measurement of the $\operatorname{PrB}_{6}$ single crystal.

a delta-peak. The width of this intensity distribution is related to the mosaicity, which corresponds to the mean variation of the principle axis within the crystal [79]. For the present $\operatorname{PrB}_{6}$ crystal the width of the rocking-curve is about $0.03^{\circ}$, which is the resolution limit of the diffractometer. Consequently, the principle axis are well aligned and a high crystal quality is present.

In the following measurements, the crystal position is moved with respect to the gamma-beam to find out whether the (100)-plane shows a torsion or a tilting along the crystal. Figure 4.1 (c) shows the torsion measurement of the $\operatorname{PrB}_{6}$ single crystal. Here, a given $\omega$-range is examined and the mean (100)-peak position is recorded for each chosen crystal position. As seen, the torsion throughout the crystal is $0.16^{\circ}$, which is negligibly small in the scope of our sample preparation. The tilting shown in Fig. 4.1 is even smaller, only about $0.04^{\circ}$ throughout the whole crystal. In summary, the present $\operatorname{PrB}_{6}$ crystal had an excellent quality. For a scheme of how torsion and tilting are examined, see Fig. 4.2. 


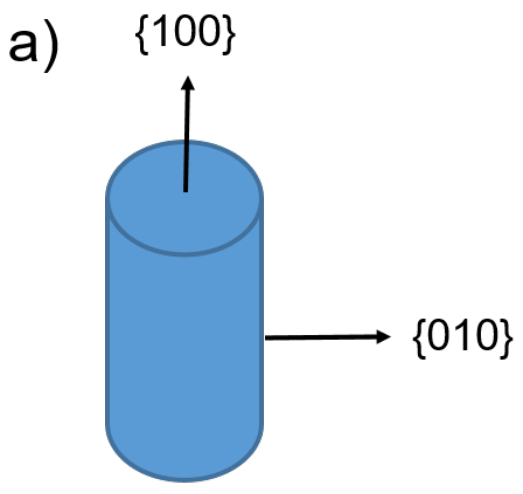

$\omega$ b)

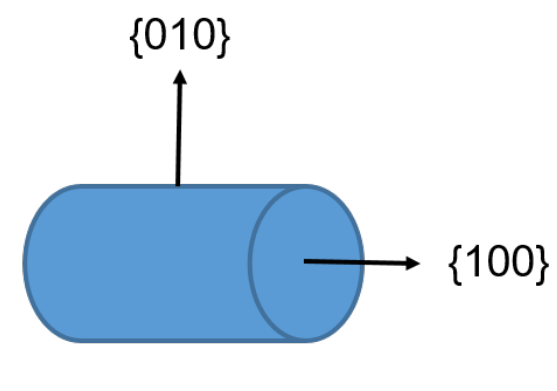

$\omega$

Figure 4.2: a) A torsion measurement is performed by rotating the crystal along the principle (100) axis and recording the (010) position on the crystal. Subsequently, another crystal position along the (100) axis, here called relative crystal position, is examined. If the (010) peak positions are not found at the same rotational angle $\omega$, a torsion is present. b) For a tilt measurement, the crystal is turned by $90^{\circ}$ and the rotational angle $\omega$ is around the (010) axis. The translation of the crystal along the principle (100) axis.

In the following, the $\mathrm{LaB}_{6}$ crystal is examined. Figure 4.3 shows a rockingcurve of the investigated $\mathrm{LaB}_{6}$ crystal and a position dependent measurement. The rocking-curve shown in Fig. 4.3 (a) has a width of about $0.14^{\circ}$, which is notably worse than the resolution limit. Additionally, the (100)-signal disappeared for certain crystal positions, as shown in Fig. 4.3 (b). Here, the intensity at the detector is counted at a given $\omega$ along the crystal. For measurement 1 only the first and the last few mm show a drop of intensity. For measurement 2 the crystal was rotated by $90^{\circ}$ and no signal of the (100)-planes could be detected for a region of nearly $10 \mathrm{~mm}$. Consequently, the lower part of the crystal was cut off and was not further processed within the sample preparation. The remaining upper part on the other hand was sufficiently good for our sample preparation and was used in our STM experiments.

The gamma-ray experiments were partly conducted within the scope of the bachelor thesis of Lisa Harmsen [29]. 

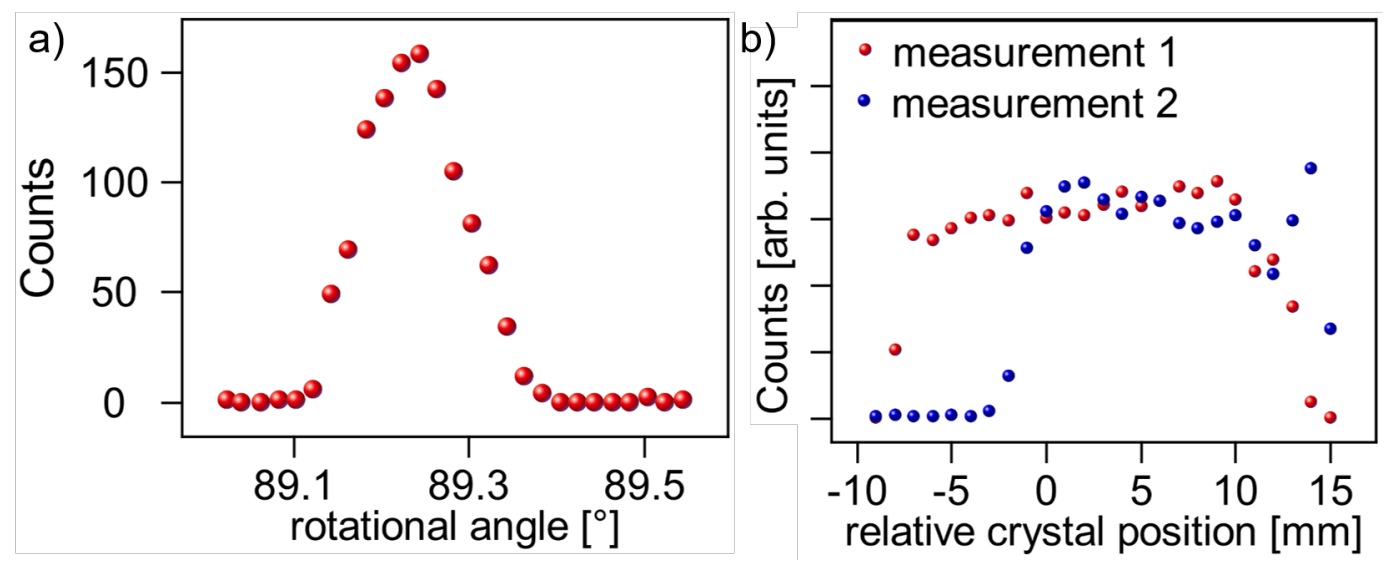

Figure 4.3: a) Rocking-curve of a $\{100\}$-peak of $\mathrm{LaB}_{6}$. b) Two measurements of perpendicular $\{100\}$-peaks. The relative crystal position is along the principle (100) axis. The measured intensity for a $\{100\}$-peak was almost vanishing for over few mm, see measurement 2. 


\section{Chapter 5}

\section{Scanning tunneling microscopy}

The main results of this thesis are scanning tunneling microscopy and spectroscopy investigations of the $\mathrm{LaB}_{6}$ and $\mathrm{PrB}_{6}$ (001)-cleavage planes. To provide an easier access to these studies for readers who are not familiar with these techniques, a brief description of the working principle and the measurement modes are given. A thorough introduction to STM, its applications and a detailed derivation of the following equations can be found in the books of Chen [17] and Voigtländer [101].

The STM is the Nobel Prize awarded experimental realization of atomic scale imaging of surfaces [69]. Although exploration of the atomic scale of surfaces has been already achieved via electron diffraction in reciprocal space beforehand [41], the pioneering work of Binning and Rohrer was the real space imaging of atomic surface structures. Actually, by probing the surface structure the local density of states (LDOS) is mapped [95]. Hence, STM is a fascinating technique to explore both surface morphology and electronic structure.

The fundamental working principle of STM is the quantum mechanical tunneling effect. Here, the orbitals of conductive surface atoms and a sharp metal tip begin to overlap when they are brought close together. By applying a bias voltage between sample and tip a tunneling current starts to flow. High spatial resolution in the sub-Å regime can be achieved since the tunneling current varies exponentially with the sample-tip distance. The tip height $z$ above the sample is adjusted by piezo crystals via a feedback loop. The tip can be positioned laterally across the surface by additional x- and y-piezo tubes. All the tip movements 
acquire a sophisticated setup for both noise cancellation and measurement electronics. Moreover, to avoid surface contaminations due to atmospheric gases, the STM experiments in the present work were performed under UHV conditions. A thorough description of the home-built low-temperature STM setup can be found in the work of Rosentreter [70].

\subsection{Theory}

Early tunneling experiments were conducted using planar electrodes, which were separated by an insulating oxide layer. In 1961, Bardeen [8] developed a theory for this setup, which was adapted later on for the STM geometry. The planar tunneling junction is modeled by a set of wavefunctions for each electrode using time-dependent perturbation theory. Here, tunneling is the transition from states of one elctrode, in the present case the tip, $\psi_{\mu}$, to the sample states $\psi_{\nu}$. The transition matrix $M_{\mu \nu}$ is written by

$$
M_{\mu \nu}=-\frac{\hbar^{2}}{2 m} \int\left[\psi_{\mu}^{*} \nabla \psi_{\nu}-\psi_{\nu} \nabla \psi_{\mu}^{*}\right] \mathrm{d} \vec{S}
$$

The surface between the states $\psi_{\mu / \nu}$ is labeled as $\vec{S}$. By applying a bias voltage $V_{B}$ a tunneling current $I_{T}\left(V_{B}\right)$ flows:

$$
\begin{array}{r}
I_{T}\left(V_{B}\right)=\frac{2 e \pi}{\hbar} \sum_{\mu, \nu}\left[f\left(E_{\mu}\right)-f\left(E_{\nu}\right)\right]\left|M_{\mu \nu}\right|^{2} \delta\left(E_{F}+V_{B}-E_{\nu}\right) \\
I_{T}\left(V_{B}\right) \approx \frac{2 \pi}{\hbar} e^{2} V_{B} \sum_{\mu, \nu}\left|M_{\mu \nu}\right|^{2} \delta\left(E_{\mu}-E_{F}\right) \delta\left(E_{F}-E_{\nu}\right)
\end{array}
$$

The Fermi-distribution is denoted by $f(E)$ and $\delta$ is the delta-distribution. This approximation is only valid for small bias voltages and low temperatures. The summands in the equation above are the transfer rates, which are given by Fermis golden rule. To further simplify the expression, $M_{\mu \nu}$ is considered to be a function of energy only. Hereafter, the sums turns into an integral over the density of states 
(DOS) of tip $\rho_{T}(E)$ and sample $\rho_{S}(E)$

$$
\left.I_{T}\left(V_{B}\right) \sim \int\left[f_{S}(\varepsilon-e V)-f_{T}(\varepsilon)\right] \rho_{S}(\varepsilon-e V) \rho_{T}(\varepsilon)\right]\left|M_{\mu \nu}\right|^{2} \mathrm{~d} \varepsilon
$$

Tersoff and Hamann [95, 94] made additional specifications for Bardeens approach to meet the actual STM geometry. The tip wave function is modeled by a s-wave function centered at the tip apex $\overrightarrow{r_{0}}$. For small bias voltages and low temperatures the tunneling current is proportional to the LDOS of the sample $\rho_{S}$

$$
I_{T}(V) \sim V \rho_{S}\left(E_{F}, \overrightarrow{r_{0}}\right)
$$

However, in actual tunneling experiments the bias voltage exceeds easily the $\mathrm{mV}$ range up to $\sim 2 \mathrm{~V}$. Hamers [28] realized, that under these assumptions $\left|M_{\mu \nu}\right|^{2}$ is not a constant anymore. The transmission $T(\varepsilon)$ becomes a function of energy and sample-tip distance using the WKB approximation for the tunneling barrier:

$$
T(\varepsilon)=\exp \left(\frac{2 z \sqrt{2 m}}{\hbar} \sqrt{\frac{\Phi_{S}+\Phi_{T}}{2}+\frac{e V}{2}-\varepsilon}\right)
$$

The work function of tip and sample are denoted by $\Phi_{T}$ and $\Phi_{S}$, respectively. Using the expression for the transmission, the tunneling current reads

$$
\begin{array}{r}
I_{T}(V) \sim \int\left[f_{S}(\varepsilon-e V)-f_{T}(\varepsilon)\right] \rho_{S}(\varepsilon-e V, r) \rho_{T}(\varepsilon, r) T(\varepsilon, e V, z) \mathrm{d} \varepsilon \\
\approx \int_{0}^{e V} \rho_{S}(\varepsilon-e V, r) \rho_{T}(\varepsilon, r) T(\varepsilon, e V, z) \mathrm{d} \varepsilon
\end{array}
$$

\subsection{Measurement modes}

The STM can be used to explore much more than just the surface morphology. Throughout the years of operation various measurement techniques have been developed, such as multi-bias imaging, apparent barrier height measurements or scanning tunneling spectroscopy (STS). Since these techniques were used in the present thesis, a brief explanation of their operation principle and their usage will be given in the following. 


\subsubsection{Constant current topography}

One of the most commonly used STM measurement technique is the constant current topography (CCT). Here, a bias voltage is applied to the sample and the tip height is adjusted via a feedback loop to maintain the desired tunneling current set point. To obtain a CCT image, the tip is moved across the surface. Firstly, the tip is moved along a straight line in the so-called fast scan axis, $x$ coordinate, and backwards in the following. The resulting tip height is recorded as each trace, forward scanning direction, and retrace, backward scanning direction. Secondly, the tip is moved by an incremental step in the $y$ direction, called slow scan axis respectively. Repetitively, the recording of trace and retrace continues and subsequently the tip moves increment by increment in $y$ direction. As described beforehand, the tunneling current is under certain assumptions proportional to the LDOS. Therefore, a measured topographic protrusion originates either from a structural elevation such as an atomic step, or a modulation of the LDOS.

\subsubsection{Multi-bias imaging}

The goal of multi-bias imaging is to map the variations in the LDOS at several different bias voltages. By changing the bias voltage, different surface orbitals can be addressed. Therefore, the corrugation difference between occupied and unoccupied states can be mapped by reversing the bias polarisation. However, comparing CCTs that are recorded after each other can be misleading. Since a thermal drift or non-linear piezo movements are present in actual experiments, the starting position of the first and second CCT are not necessarily the same. Oftentimes this leads to an additional shift in the atomic corrugation, which is erroneously superimposed with the mapped LDOS. To avoid this artifact, the CCTs of a multi-bias image are recorded quasi-simultaneously. Here, each fast-axis line scan is recorded with the desired set-points after each other, before continuing with the next line in the slow-scan axis. Since the same line is scanned shortly after each other, the thermal drift is minimized and the aforementioned additional shift can be neglected for the data interpretation. By changing the applied bias voltage the relative tip height $\Delta z$ may changes as well, as seen in Fig. 5.1. A sudden decrease in tip height can be a hint towards a smaller LDOS or faster decaying 
electronic states within this bias voltage range. Another way to stimulate the tip height is changing the current set point. Hence, a multi-bias data set consists of quasi-simultaneously recorded CCT images at various bias voltages or tunneling current set-points.
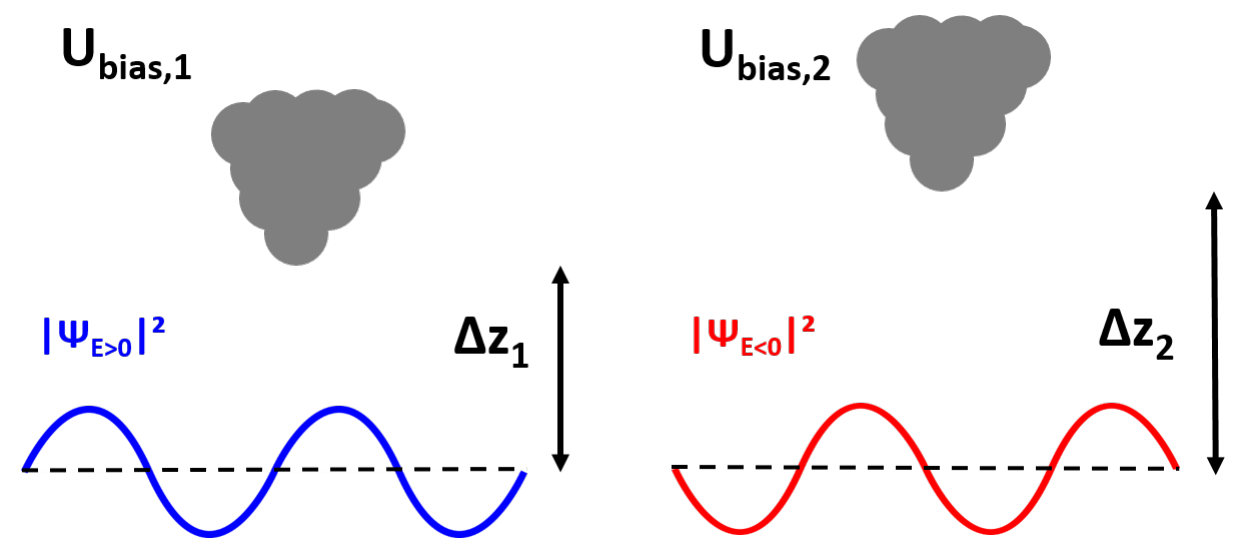

Figure 5.1: Scheme of a multi-bias imaging process. On the left, $\mathrm{U}_{\mathrm{Bias}, 1}$ is applied, which addresses the unoccupied states and on the right $U_{\text {Bias, } 2}$, which addresses the occupied states. The corrugation changes from left to right as well as the average tip height $\Delta z$ above the surface.

\subsubsection{Apparent barrier height}

In a standard model the tunneling current drops exponentially with the tip-sample distance $d$ via

$$
I_{T}=e^{-2 \kappa d}
$$

The decay constant $\kappa$ contains the so-called apparent barrier height $\Phi_{\text {app }}$.

$$
\kappa=\frac{\sqrt{2 m \Phi_{\mathrm{app}}}}{\hbar}
$$

$\Phi_{\text {app }}$ can be interpreted as the effective barrier seen by the tunneling electron between sample and tip. Technically, it can be calculated by measuring the ratio of two tunneling currents $I_{1} / I_{2}$ at two different tip-sample distances $d_{1}$ and $d_{2}$ via 
[17]

$$
\Phi_{\mathrm{app}}=\frac{\hbar^{2}}{8 m}\left(\frac{\ln \left(I_{1} / I_{2}\right)}{\Delta d}\right)^{2}
$$

Experimentally, the tip is moved closer to the surface by typically $0.5 \AA$ to $1 \AA$ and the resulting current is recorded at the fixed bias voltage set point. Since the current difference is mapped for a given bias voltage, $\Phi_{\text {app }}$ effectively maps the decay behavior of the addressed surface wave function. If the addressed wave function is slowly decaying into the vacuum, the difference in tunneling current will be rather small for a given tip-sample distance change. Hence, the calculated value of $\Phi_{\text {app }}$ will be rather small. By varying the bias voltage different states can be addressed and $\Phi_{\text {app }}$ may depends on the bias voltage.

\subsubsection{Scanning tunneling spectroscopy}

Another powerful technique to map the electronic surface structure is scanning tunneling spectroscopy (STS). Here, an $I(V)$ curve is taken at a desired measurement position $(x, y)$. After adjusting the tip height for the given set-point, the feedback-loop is paused. The voltage is subsequently modulated and the measured current is recorded as an $I(V)$-curve. The resulting differential conductance $\mathrm{d} I / \mathrm{d} V(V)$ is, under certain assumptions, proportional to the $\operatorname{LDOS}(V)$ [28]. The derivative of $I(V)$ can be obtained either numerically or by using a lock-in technique. By taking an $I(V)$ curve at measurement points of a CCT image, it is possible to resolve the differential conductance laterally. Since the outcoming data set is multi-dimensional, several visualization techniques are commonly used.

a) Single spectrum At a certain position $(x, y)$ a $\mathrm{d} I / \mathrm{d} V(V)$ curve is obtained. The resulting $\mathrm{d} I / \mathrm{d} V(V)$-values are plotted against the applied bias voltage $V$ as a simple graph.

b) Spectrum cross section Here, the $\mathrm{d} I / \mathrm{d} V(V)$ behavior is displayed as a three dimensional data set along a certain spatial cross section. Typically, the spatial coordinate is plotted as $\mathrm{x}$-axis and the applied bias voltage is presented as $y$-axis. The magnitude of the differential conductance is depicted 
color-coded.

c) Spectrum map The $\mathrm{d} I / \mathrm{d} V(V)$ values at a certain bias voltage $V_{0}$ can be visualised as a spectrum map. The spatial coordinates $(x, y)$ remain as $\mathbf{x}$ and $\mathrm{y}$-axis, and $\mathrm{d} I / \mathrm{d} V\left(V_{0}\right)$ is plotted color-coded.

\subsubsection{Topography normalization}

If the differential conductance is recoded quasi-simultaneously with a CCT image, the $I(V)$-curves are subsequently recorded on top of the modulated contour line given by the CCT. At the set point, no modulation of the current should be present, as seen in Fig. 5.2. For each depicted lateral displacement, light green or dark green spot, the measured current remains the set point value. This scenario might change if the bias voltage polarity is switched. The corrugation maxima of the wave function might shift for the unoccupied side, as seen for the red curve in Fig. 5.2. At the light green marked location, where the corrugation minimum is situated for $E<0$, the measured current is disproportionally small due to the tip height. At the dark green position, the tip is closest to the surface, which coincides with a corrugation maximum for the unoccupied states. Consequently, the measured current is disproportionally large, as seen in the right hand side of Fig. 5.2.

To avoid these aforementioned measurement artifacts, the tip height can be projected onto a constant height above the surface. To do so, the vertical distance $\Delta z(x, y)$ from the nominal tip height average is calculated for each position $(x, y)$. By using the apparent barrier height, the normalized current values can be calculated via

$$
I_{\text {norm }}=I_{\text {prenorm }} e^{+2 \kappa(x, y) \Delta z(x, y)}
$$

The outcome of this transformation is depicted schematically in Fig. 5.3. After the topography normalization, the obtained current for the set point, here at positive voltage, is modulated according to the respective wave function. At the negative voltage flank, the disproportionate mapping of the current is minimized.

The concept of topography normalization has been introduced by Jens Garleff 


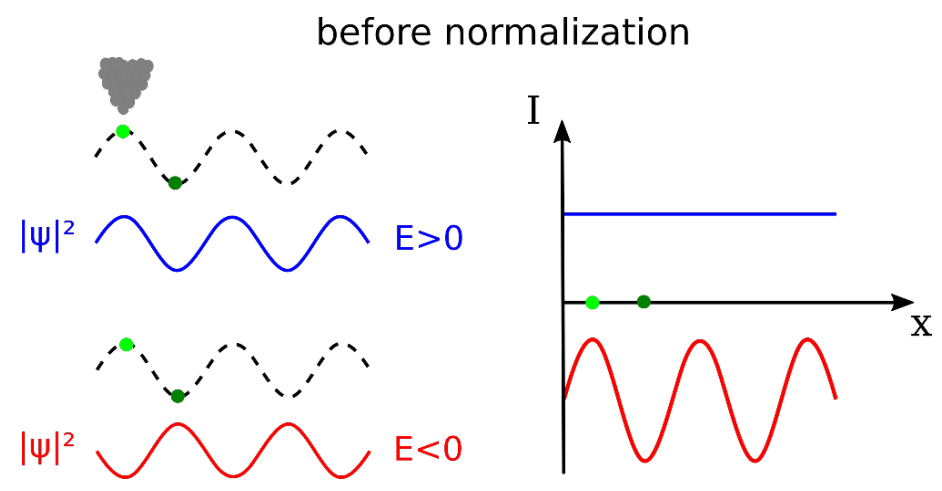

Figure 5.2: At the set-point of a CCT the outcoming $I$ signal is the set-point and therefore constant, blue curves. If the corrugation changes by bias voltage switching, red curves, the resulting $I$ signal might be distorted. The tip height above the surface is marked as a dotted line and the light and dark green spots symbolizing different tip positions. The figure has been reprinted from [11].

and additional details can be found in Ref. [23].

\section{after normalization}
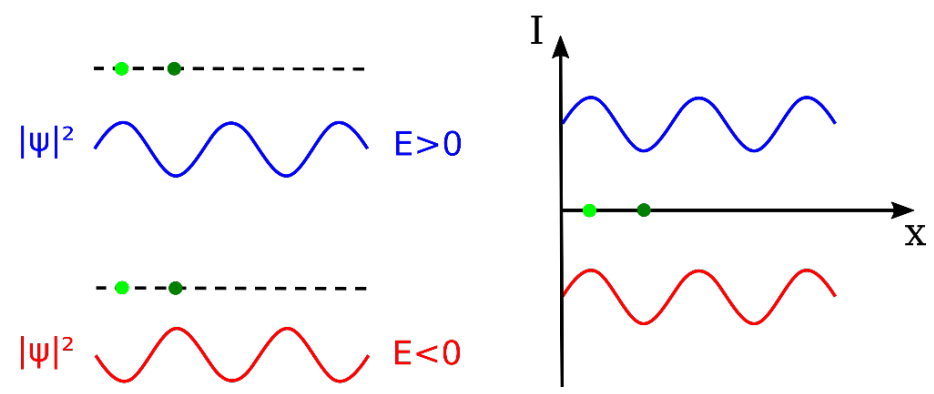

Figure 5.3: After the topography normalization the current modulation artifacts are corrected. The color-code is the same as in Fig. 5.2. The figure has been reprinted from [11]. 


\section{Chapter 6}

\section{Spatially resolved low-energy electron diffraction}

A complementary technique to explore the surface structure is low-energy electron diffraction (LEED). Here, an electron beam is directed to the sample, where it can be diffracted by the surface lattice planes. The resulting diffraction pattern allows to draw conclusions regarding the surface structure, which can severely differ from the bulk periodicity due to surface reconstruction [44]. Since the surface has a reduced dimensionality compared to the bulk, the two dimensional diffraction equation reads:

$$
n \lambda=d_{2 D} \sin (\Phi)
$$

In Fig. 6.1 a scheme is presented for surface diffraction. The incoming electrons are denoted by a down-arrow and the elastically back-scattered electrons by the up-arrow at an angle of $\Phi$. For constructive interference of the scattered electrons the path difference $\delta$ has to be an integer multiply $n$ of the electron wavelength $\lambda$. Here, $d_{2 \mathrm{D}}$ denotes the distance between the lattice planes in two dimensions.

The incoming electrons have energies from about $50 \mathrm{eV}$ to $200 \mathrm{eV}$ and for a typical energy of $100 \mathrm{eV}$ they have a penetration depth of only the first few atomic layers [50]. Therefore, in actual experiments the finite penetration depth of the electrons cannot be neglected and diffraction spots originating from the bulk periodicity can occur. Moreover, the electron bombardment of the sample 


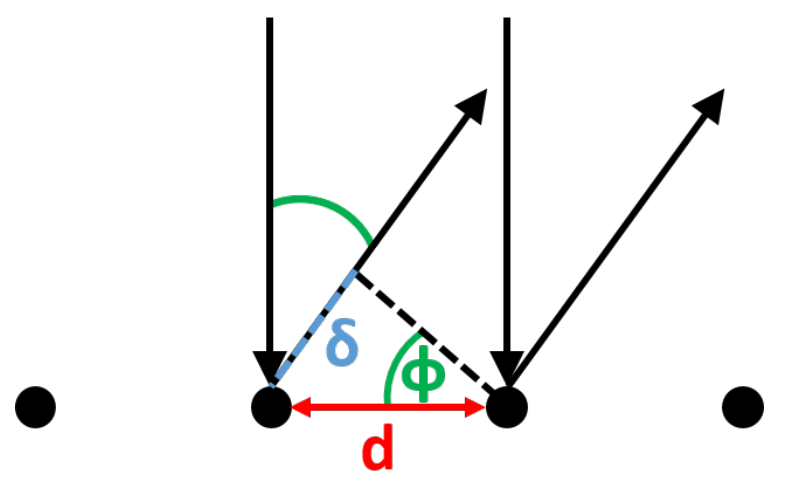

Figure 6.1: Scheme of the Bragg condition in two dimensions, adapted and modified for reflective geometry from [105]. The lattice planes are seperated by $d$, the path difference is $\delta$ and the diffraction angle is $\Phi$.

can cause electron beam induced surface damage. Additionally, in conventional LEED systems the electron beam diameter has a typical size of $1 \mathrm{~mm}$ [50]. If ordered surface domains extend on a much smaller length scale, they might be impossible to detect in conventional setups.

These practical limitations have been largely overcome by introducing a novel setup, namely the ultrafast LEED (ULEED) [99]. The term ultrafast refers to the possibility of time-resolved investigations of structural and electronic processes at surfaces, which were not used in the following experiments. Here, we employed the ULEED for spatially resolved LEED.

As electron source a tungsten nano-tip is used, which emits electrons by laser excitation. With this setup, fewer electrons hit the sample surface compared to a conventional setup, which greatly reduces the probability of electron induced surface damage. The outcoming small number of diffracted electrons are collected by a multichannel plate (MCP). The resulting electron beam has a diameter of only about $80 \mu \mathrm{m}$. Consequently, the electron beam does not average over the whole sample surface, but allows spatial resolution. Additionally, the ULEED setup is equipped with a flow cryostat, which allows cooling of the sample.

The ULEED setup has been developed in the group of Prof. Claus Ropers at the University of Göttingen and with this setup several measurements were performed on the hexaboride systems and the results are discussed in Section 9.6. 


\section{Chapter 7}

\section{Surface resonance of the $(2 \times 1)$ reconstructed lanthanum hexaboride (001)-cleavage plane: A combined STM and DFT study}

The wellspring of experiments on hexaboride surfaces was motivated by the usage of $\mathrm{LaB}_{6}$ and $\mathrm{CeB}_{6}$ as cathode materials. These materials host a surprisingly low work function by simultaneously showing a high chemical stability at elevated temperatures. Lafferty [40] was the first one to point out these superior cathode properties by reporting a work function of $2.66 \mathrm{eV}$ for polycrystalline $\mathrm{LaB}_{6}$. Consequently, the question arose, which crystal face possesses the lowest work function. After the tremendous progress in growing large single crystals, the (001) surface was found to be the one with the lowest work function [54]. To explain the mysteriously low work function it was the goal to find out the atomic structure of the (001)-surface. As shown above, LEED is a well-established technique to investigate surface structures. In 1977, measured LEED pattern of the (001)-surface showed a simple $(1 \times 1)$ symmetry [55], so a complete La or $\mathrm{B}_{6}$ termination seemed likely. Auger-electron spectroscopy (AES) [88], X-ray photoelectron spectroscopy [5] and angle-resolved AES [15] suggested a La terminated surface. Therefore, an intuitive model suggested that the low work function 
7. Surface resonance of the $(2 \times 1)$ reconstructed lanthanum hexaboride (001)-cleavage plane:

A combined STM and DFT study

originates from positively charged La ions at the surface. These La ions create a dipole moment, which in turn help the electrons to leave the surface [54].

Of course, STM is perfectly suited to access the atomic structure of surfaces and check the above stated hypothesis. STM results showed a simple $(1 \times 1)$ structure with the interatomic spacing of the bulk lattice constant $[58,59]$. The authors concluded, that the surface is, as suggested beforehand, La terminated. However, the surface was found to have a high density of defects with about $10 \%$ of the La sites to be vacant. It is worth noting, that all the observed surface steps were of one bulk lattice constant height, which in turn suggests only one surface termination. Furthermore, the steps were straight and parallel to each other.

In the following publication, we present a major revision of the previously displayed ideas of over 40 years of $\mathrm{LaB}_{6}$ surface research. Already in 2018, Schmidt et al. [74] pointed out using density functional theory (DFT) calculations, that a $(2 \times 1)$ reconstructed La terminated surface is the geometrical ground state of the $\mathrm{LaB}_{6}$ (001)-cleavage plane and not a uniform La termination. Again, the previously reported experimental results have been made on heated samples, whereas the theoretical predictions by Schmidt et al. [74] refer to cleaved samples. We insert the missing puzzle piece by performing a STM study on in situ cleaved $\mathrm{LaB}_{6}$ (001). As diplayed in detail below, a chainlike $(2 \times 1)$ reconstruction is indeed present. Atomically resolved STS showed a peak in the differential conductance below $E_{\mathrm{F}}$. In a combined approach with our DFT collaborators F. Sohn and P. E. Blöchl, this peak is identified as a surface resonance of the $(2 \times 1)$ reconstruced surface. However, the $(2 \times 1)$ reconstruction is rather labyrinth-like arranged. Only in the vicinity the step edges, the La rows tend to order next to each other. This spatial confinement explains why we have not found signals of a $(2 \times 1)$ reconstruction in our conventional LEED setup. Therefore, we performed additional LEED experiments using the ultrafast setup displayed in Chapter 6. Here, the $(2 \times 1)$ reconstruction spots could be observed on some of the sample areas.

In conclusion, we proved the hypothesis of Schmidt et al. [74] and showed additionally that the surface electronic structure severely differs from the bulk band structure.

The following Section 7.1 has been published as article in Phys. Rev. B 
100, 205407 (2019), DOI: 10.1103/PhysRevB.100.205407. The publication was mainly written by P. Buchsteiner and F. Sohn. The STM experiments were carried out by J. Voigt within his master thesis [100] and by P. Buchsteiner. STM data interpretation and analysis were done by J. Voigt, P. Buchsteiner and M. Wenderoth. The DFT results were obtained by F. Sohn and P. E. Blöchl. J. G. Horstmann performed the ULEED measurements under the supervision of $\mathrm{C}$. Ropers. The $\mathrm{LaB}_{6}$ single crystals were grown by M. Ciomaga Hatnean and G. Balakrishnan.

Minor adaptions were made for the reprint of the article, for example order of references, enumeration of figures and sections, changes of font and font size.

\subsection{Original publication}

We performed a combined study of the (001)-cleavage plane of lanthanum hexaboride $\left(\mathrm{LaB}_{6}\right)$ using scanning tunneling microscopy and density-functional theory (DFT). Experimentally, we found a $(2 \times 1)$ reconstructed surface on a local scale. The reconstruction is only short-range ordered and tends to order perpendicularly to step edges. At larger distances from surface steps, the reconstruction evolves to a labyrinthlike pattern. These findings are supported by low-energy electron diffraction experiments. Slab calculations within the framework of DFT shows that the atomic structure consists of parallel lanthanum chains on top of boron octahedra. Scanning tunneling spectroscopy shows a prominent spectral feature at $0.6 \mathrm{eV}$. Using DFT, we identify this structure as a surface resonance of the $(2 \times 1)$ reconstructed $\mathrm{LaB}_{6}(\mathbf{1 0 0})$ surface which is dominated by boron dangling bond states and lanthanum $d$ states.

P. Buchsteiner, F. Sohn, J. G. Horstmann, J. Voigt, M. Ciomaga Hatnean, G. Balakrishnan, C. Ropers, P. E. Blöchl, M. Wenderoth 
7. Surface resonance of the $(2 \times 1)$ reconstructed lanthanum hexaboride (001)-cleavage plane:

A combined STM and DFT study

\section{Introduction}

The rare-earth hexaborides $\left(R \mathrm{~B}_{6}\right)$ are a material class with a common, relatively simple crystalline structure, but widely tunable electronic and magnetic properties. For example, dense Kondo behavior is found in $\mathrm{CeB}_{6}$ [90], $\operatorname{PrB}_{6}$ and $\mathrm{NdB}_{6}$ order antiferromagnetically [24], $\mathrm{SmB}_{6}$ is a Kondo insulator [37, 82], $\mathrm{EuB}_{6}$ is a ferromagnetic semimetal which exhibits colossal magnetoresistance [65], and $\mathrm{YbB}_{6}$ is proposed to host topologically protected states without a Kondo mechanism [53]. The variety of these phenomena can be traced back to the $4 f$ occupancy increasing from $4 f^{0}$ for La up to $4 f^{14}$ in $\mathrm{Lu}$ as the rare-earth elements.

All rare-earth hexaborides share the same cubic crystal structure with the $\mathrm{B}_{6}$ octahedra located at the cube's corners and the rare-earth element at the center, as seen in Fig. 7.1 (a). A three-dimensional covalent binding network between the $\mathrm{B}_{6}$ octahedra can be achieved by electron donation of the rare-earth element, leading to positively charged ions and negatively charged boron cages in the crystal structure [43]. The lattice constant changes only slightly across the $R \mathrm{~B}_{6}$ series.

One of the most prominent hexaborides is $\mathrm{LaB}_{6}$, a widely used electron emitter due to its extraordinarily low work function [96]. Recently there has been a growing interest in its solar heat absorbance with regard to possible applications in solar energy devices [73, 48]. Although surface properties play a crucial role in these applications, surface studies of this system display a rather incomplete picture. Even the ground state geometrical structure of the (001) surface is under current debate. Up to the present date, experimental results show a simple $(1 \times 1)$ reconstructed surface [96], as found in low-energy electron diffraction (LEED) and Auger electron spectroscopy studies [55, 5, 4, 89, 88, 15, 25]. Scanning tunneling microscopy (STM) experiments at room temperature and under UHV conditions have shown a $(1 \times 1)$ structure, which has been described to be lanthanum terminated $[58,59]$. However, it should be noted that the samples of the aforementioned studies have been prepared by polishing and heating. Recently, surface slab calculations of $\mathrm{LaB}_{6}(001)$ based on density functional theory (DFT) have been made [74]. Therein, various surface reconstructions are taken into account. Their prediction is a $(2 \times 1)$ reconstructed surface as ground state. These findings seemingly contradict the $(1 \times 1)$ surface structure observed so far. 


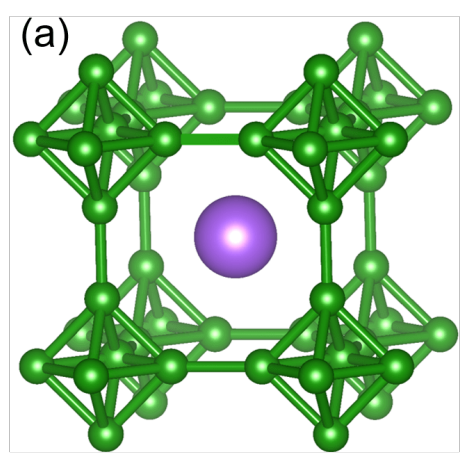

(b)
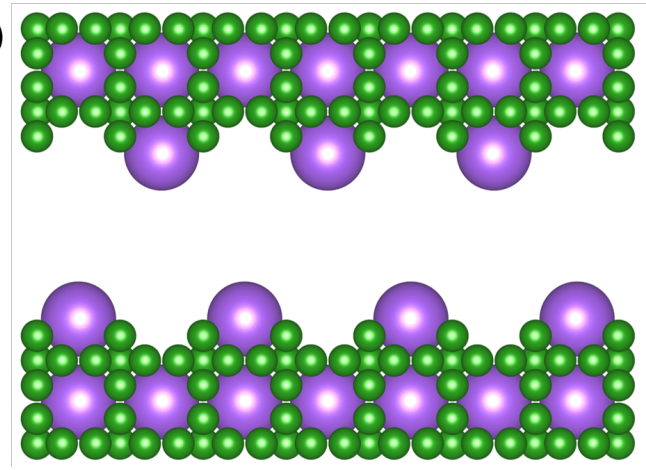

Figure 7.1: (a) Crystal structure of $R \mathrm{~B}_{6}$, where the rare-earth element is located at the center of the simple cubic unit cell and the $\mathrm{B}_{6}$ octahedra at the corners. (b) Side view of two cleaved surfaces, where each side is partly La terminated.

To solve this discrepancy between previous experimental results and recent theoretical predictions, we investigated $\mathrm{LaB}_{6}(001)$ prepared by cleavage in UHV. Therefore, annealing-related impacts on the surface morphology, such as preferential evaporation or thermally activated reorganization, can be minimized.

A $(2 \times 1)$ reconstructed surface is unambiguously found by using high-resolution STM and LEED measurements. DFT slab calculations resolve the chemical nature of the $(2 \times 1)$ reconstruction as parallel rows of lanthanum atoms on top of non-reconstructed $\mathrm{B}_{6}$ cages. Its electronic structure close to the Fermi energy is governed by a surface resonance, which is mainly composed of boron $s p$-hybrid dangling bond orbitals. 
7. Surface resonance of the $(2 \times 1)$ reconstructed lanthanum hexaboride (001)-cleavage plane:

A combined STM and DFT study

\section{Methods}

\section{Experimental techniques}

STM experiments were performed in a home-built microscope operating at $8 \mathrm{~K}$ and at a base pressure of $4 \times 10^{-11}$ mbar. Tunneling tips were made by electrochemical etching of polycrystalline tungsten wire. The $\mathrm{LaB}_{6}$ single crystals were grown using the floating zone technique as described in Refs. [6, 7]. The crystals were oriented by gamma-ray diffraction and cut along the (001) plane into rectangular samples of about $1 \times 4(\mathrm{~mm})^{2}$ size and $300 \mu \mathrm{m}$ thickness. The samples were cleaved insitu at room temperature along the (001) plane followed by immediate transfer to the STM head at cryogenic temperature. All STM images were recorded using the constant current topography (CCT) mode. LEED experiments were performed on identically prepared samples, cleaved at a base pressure of $2 \times$ $10^{-8}$ mbar and investigated at $2 \times 10^{-10} \mathrm{mbar}$. The diffraction images presented in this paper were recorded at either room temperature or at $27 \mathrm{~K}$. For our LEED experiments, we used an ultrafast LEED setup (ULEED), as described in Ref. [99]. This setup features a laser-pulsed electron gun with an electron beam diameter of about $80 \mu \mathrm{m}$ at the sample. With this technique, the electron beam contains significantly less electrons than in conventional systems. Thus, the possibility of electron-beam-induced surface damage is drastically reduced. The resulting small number of scattered electrons is detected with a microchannel plate. Atomic force microscopy (AFM) measurements were conducted in a commercial instrument manufactured by Agilent, which operates at ambient condition.

\section{Calculations}

Theoretical results presented in this paper are based on DFT $[32,38]$ and are obtained with the CP-PAW code [1], which employs the projector-augmented wave method [9] together with a functional minimization scheme derived from the CarParrinello molecular dynamics approach [14]. We use the local hybrid exchangecorrelation functional PBE0r described elsewhere [81], which locally replaces a fraction of the Perdew-Burke-Ernzerhof [62] exchange with the same portion of the exact Hartree-Fock exchange. In the PBE0r functional the Fock-term is ex- 
pressed in local orbitals and only on-site terms are retained.

\section{Results}

\section{Surface morphology}

For most of the encountered surfaces probed by STM, the surface appears rather disordered. This is described in more detail in Section 7.1. Atomically ordered areas on $\mathrm{LaB}_{6}$, as seen in Fig. 7.2, are scarce and have to be searched for. The atomic structure appears chain-like with a spacing of two bulk lattice constants. Hence, $(2 \times 1)$ reconstruction peaks can be clearly observed in the Fourier analysis, as seen for the red-marked area of Fig. 7.2. However, this $(2 \times 1)$ reconstruction is ordered only on a short range and is mainly labyrinthlike arranged, as seen in the upper right corner of the CCT image in Fig. 7.2. Although individual chains can still be resolved, no signs of a $(2 \times 1)$ reconstruction can by found in the Fourier analysis due to the lack of long-range order.

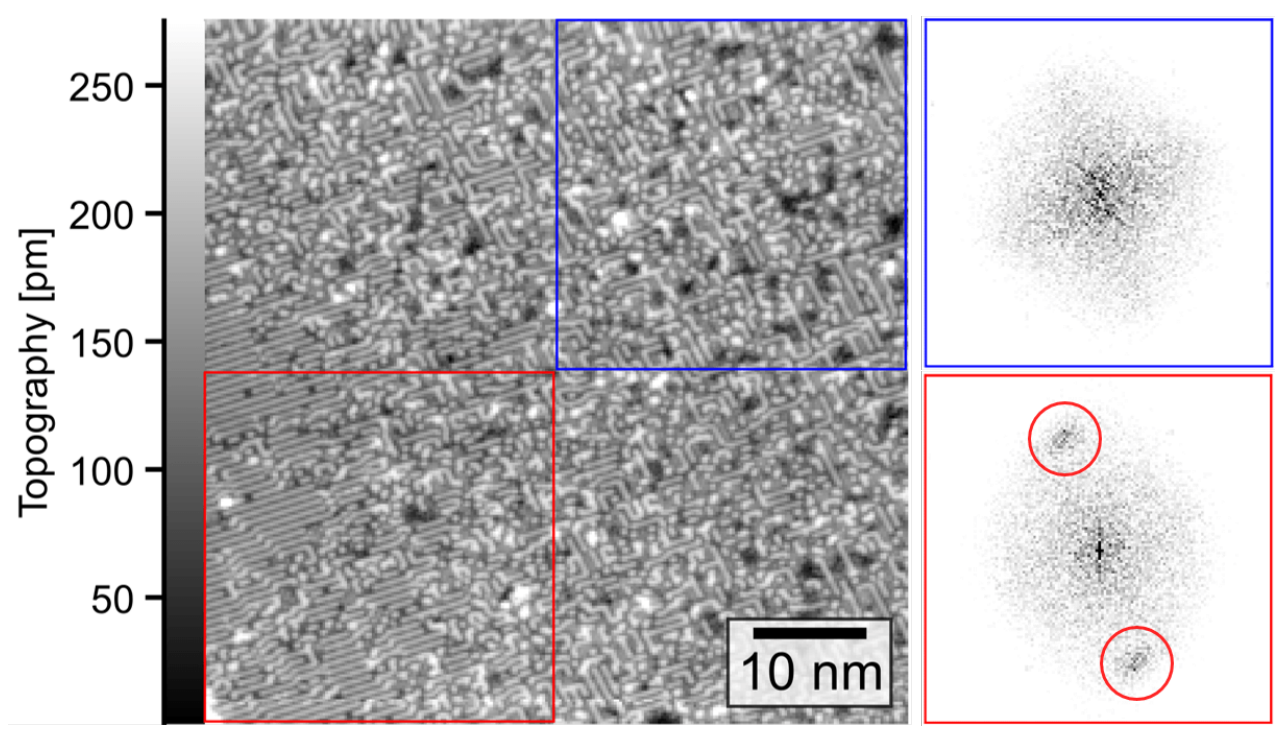

Figure 7.2: Atomically resolved CCT taken at $0.8 \mathrm{~V} / 0.1 \mathrm{nA}$. Atomic rows with a spacing of two bulk lattice constants are present, which are mostly labyrinthlike arranged. The Fourier analysis of a surface area with a rather ordered region shows clear signs of the $(2 \times 1)$ reconstruction, see red-marked area. For most regions no distinct peaks in the Fourier transform can be observed, see blue-marked area. 
7. Surface resonance of the $(2 \times 1)$ reconstructed lanthanum hexaboride (001)-cleavage plane:

A combined STM and DFT study

A high-resolution image of the $(2 \times 1)$ reconstruction, Fig. $7.3(\mathrm{a})$, shows that even for the more ordered areas, the chains exhibit kinks and defects. In the vicinity of the region shown in Fig. 7.3(a), a step edge of one lattice constant height has been found.
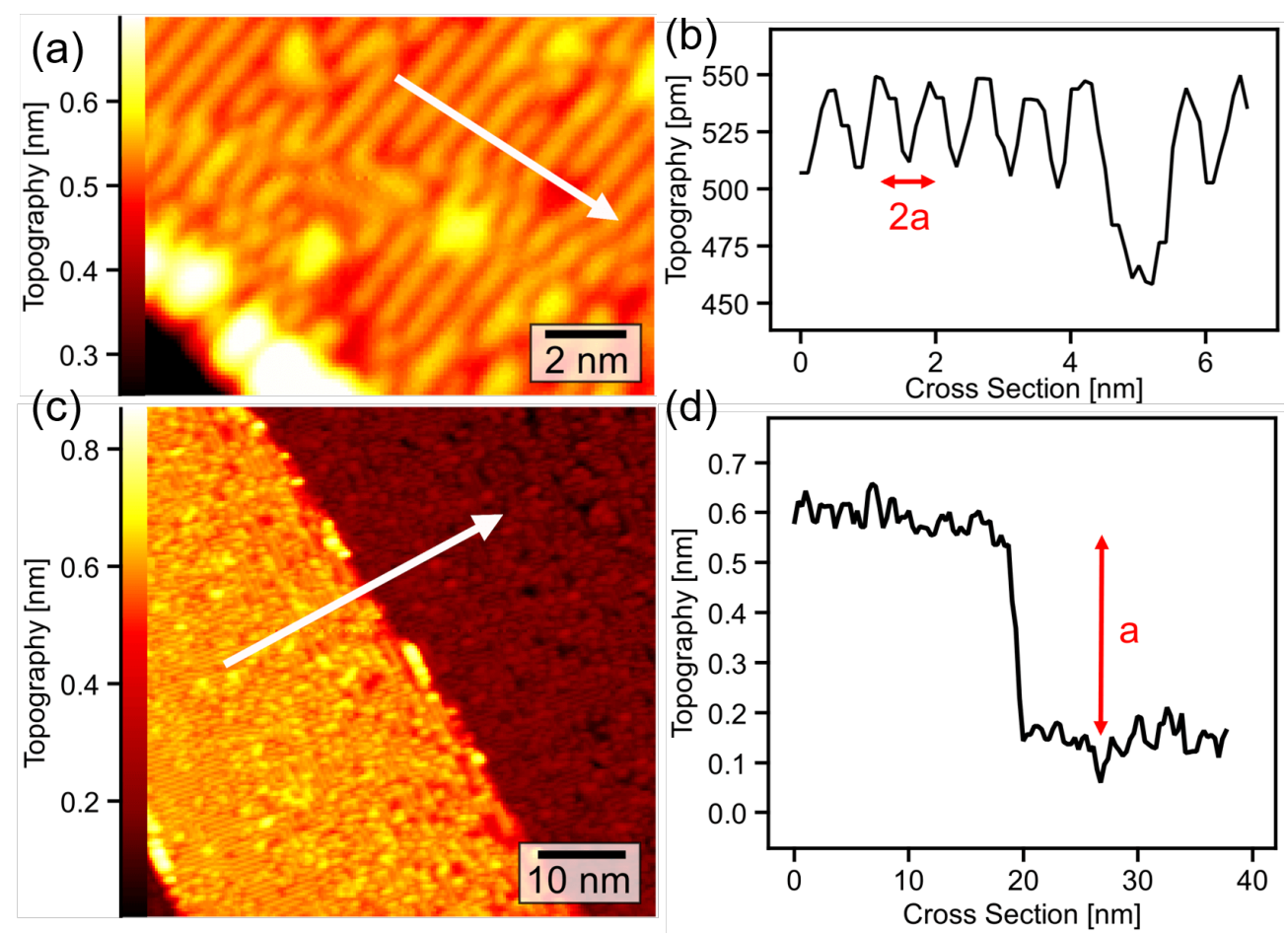

Figure 7.3: (a) High-resolution CCT of the $(2 \times 1)$ reconstruction taken at $1 \mathrm{~V} / 0.1 \mathrm{nA}$. (b) Cross section along the $(2 \times 1)$ reconstruction as indicated by the white arrow in (a). (c) Large scale overview taken at $1 \mathrm{~V} / 0.1 \mathrm{nA}$ in the vicinity of the high-resolution image in (a). (d) The height profile of (c) shows that the step is of about $4.1 \AA$ height, which amounts to one bulk lattice constant $a$.

At the step edge, the most ordered $(2 \times 1)$ reconstruction is observed. Farther away from the step, the chains lose their preferential orientation perpendicular to the step edge, and a more labyrinthlike arrangement is seen, which is similar to the observation in Fig. 7.2. Therefore, our findings so far suggest a correlation between steps and the occurrence of a well-ordered $(2 \times 1)$ reconstruction.

The spatially rather limited observation of long-range order explains why signs of a $(2 \times 1)$ reconstruction have not yet been found in LEED experiments. In our standard LEED setup, only a $(1 \times 1)$ structure could be seen that vanished 
(a)

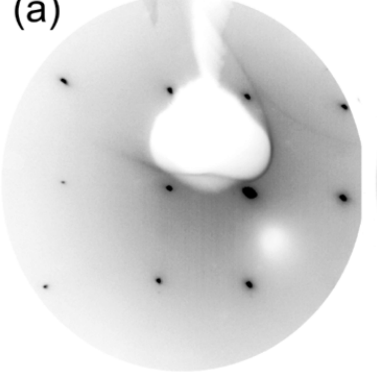

(c)

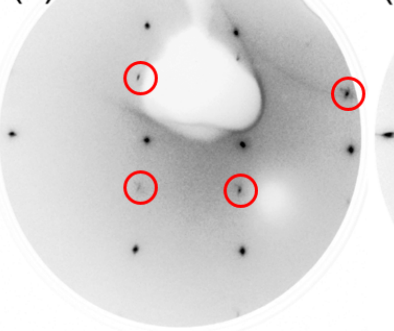

(b)

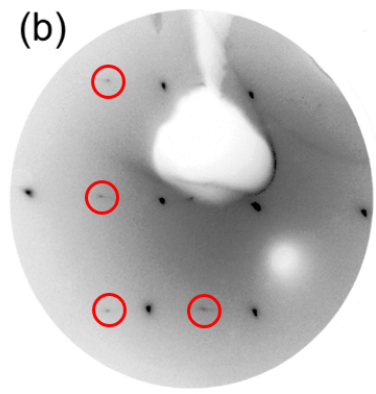

(d)

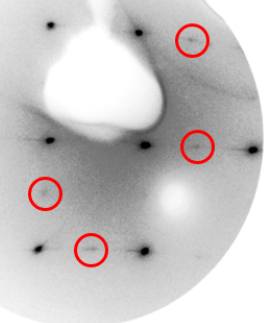

Figure 7.4: ULEED images of different locations on the cleaved $\mathrm{LaB}_{6}(001)$ surface. (a) Only $(1 \times 1)$ spots are visible, taken at $130 \mathrm{eV}$ and room temperature. In (b), additionally, $(2 \times 1)$ spots are present, $100 \mathrm{eV} / \mathrm{RT}$ and in $(\mathrm{c})(1 \times 2)$ spots are observed, $100 \mathrm{eV} / 27 \mathrm{~K}$. In $(\mathrm{d})$, both $(1 \times 2)$ and $(2 \times 1)$ spots are present, $100 \mathrm{eV} / 27 \mathrm{~K}$. The observed reconstruction spots are marked by red circles.

after about 30 minutes of measurement time at a pressure of $10^{-9}$ mbar. The situation changes when using the ultrafast LEED setup and measurements could be carried out over a couple of hours without any noticeable change. Figure 7.4 sums up the observed diffraction patterns. For most of the sample areas, only a (1 $\times 1$ ) pattern was found, as seen in Fig. 7.4 (a). In some surface areas, as shown in Fig. 7.4 (b) and Fig. 7.4 (c), signs of a $(2 \times 1)$ or $(1 \times 2)$ reconstruction are present. In one surface region, both $(2 \times 1)$ and $(1 \times 2)$ spots were observed, as depicted in Fig. 7.4 (d). Note that the diffraction patterns do not change upon cooling the sample down to $27 \mathrm{~K}$, apart from the increasing spot intensity and reduced background noise level due to the temperature-dependent Debye-Waller factor.

To clarify the chemical nature of the $(2 \times 1)$ reconstructed surface, scanning tunneling spectroscopy (STS) was carried out. 
7. Surface resonance of the $(2 \times 1)$ reconstructed lanthanum hexaboride (001)-cleavage plane:

A combined STM and DFT study

\section{Spatially resolved spectroscopy}

Figure 7.5 (a) shows a CCT image, where, simultaneously to the topography, at every measurement point an $I(V)$ curve and the apparent barrier height $\Phi_{\text {app }}$ were recorded. The chains of the $(2 \times 1)$ reconstruction in Fig. 7.5 extend for only a few unit cells and are interrupted by various defects. Using the $I(V)$ curve, its differential conductance $\mathrm{d} I / \mathrm{d} V(V)$ can be seen as an approximation for the local density of states (LDOS) [94, 95]. Figure 7.5 (b) shows two $\mathrm{d} I / \mathrm{d} V(V)$
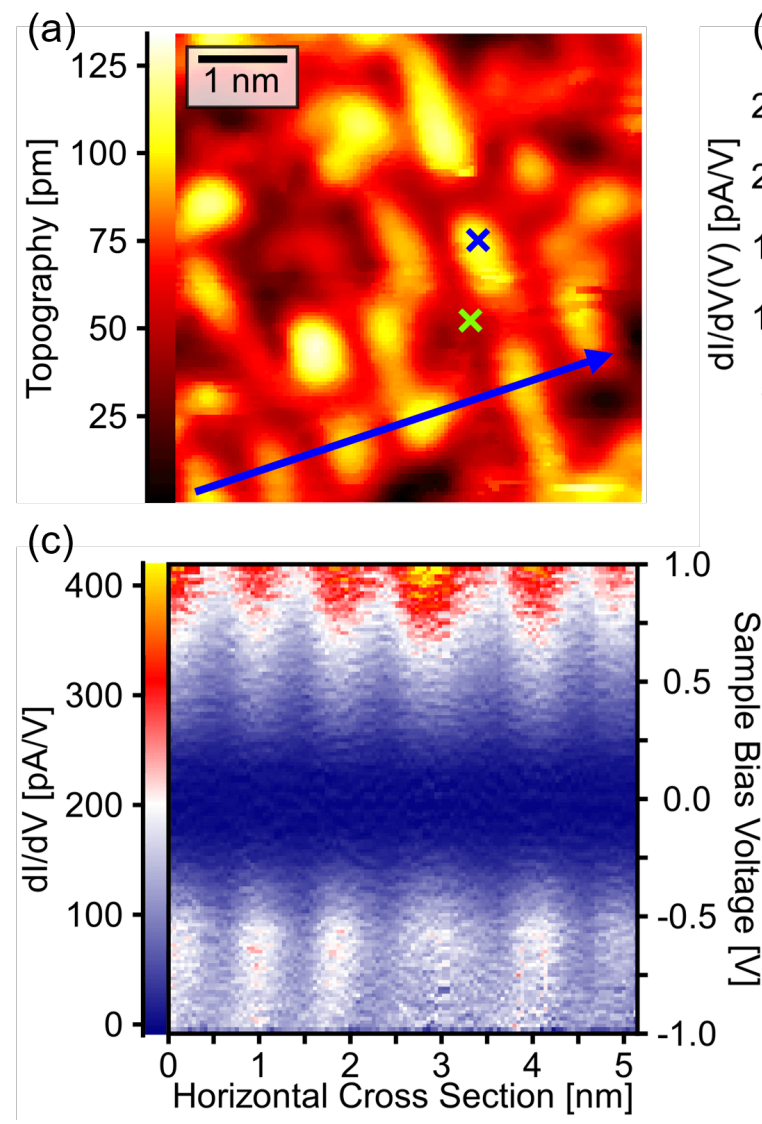

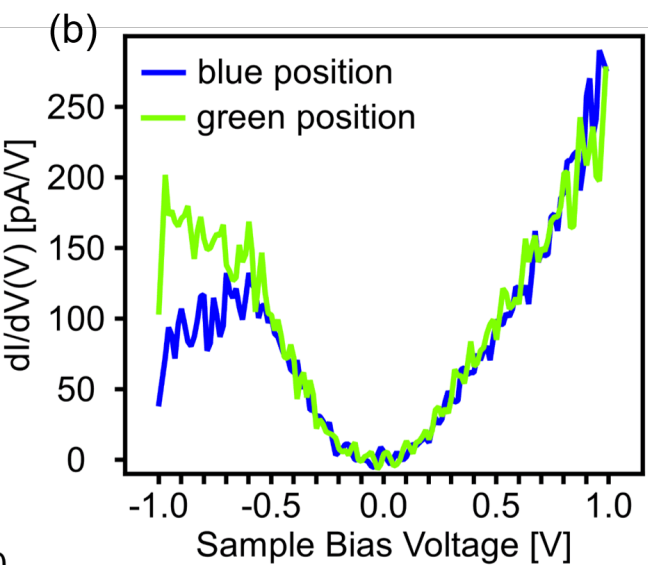

(d)

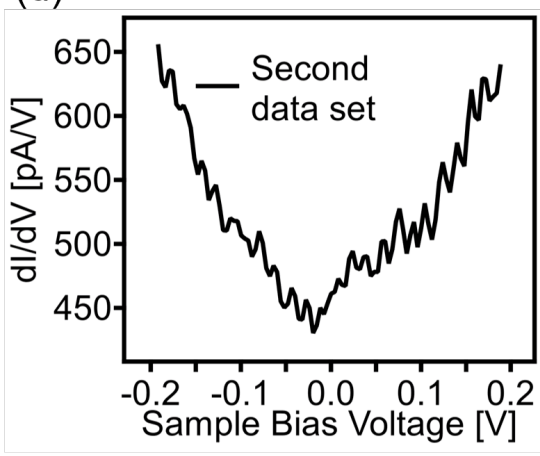

Figure 7.5: (a) CCT taken at $1 \mathrm{~V} / 0.1 \mathrm{nA}$. (b) Two exemplary $\mathrm{d} I / \mathrm{d} V(V)$ curves obtained from the marked positions are shown. (c) The horizontal arrow in (a) marks the line along the $\mathrm{d} I / \mathrm{d} V(V)$ cross section was taken, which is shown here. (d) $\mathrm{d} I / \mathrm{d} V(V)$ curve obtained from a second data set, see Section 7.1, to resolve the energy region around $E_{\mathrm{F}}$.

spectra obtained from the blue- and the green-marked positions. Clearly, a peak 
in the differential conductance at about $-0.6 \mathrm{eV}$ can be resolved on top of the protrusion of the reconstruction, blue-marked position, as well as a steep rise of $\mathrm{d} I / \mathrm{d} V(V)$ toward positive bias voltages. To visualize the spatial variation of the LDOS, the differential conductance can be plotted position dependent as a color coded $\mathrm{d} I / \mathrm{d} V(V, x)$ cross section. Since the $I(V)$ curves are recorded quasisimultaneously to the CCT, these curves are taken on a modulated contour line given by the topography. To project the measurement onto a constant height above the surface, a topography normalization is applied, as described in Ref. [23]. This can be done by using the apparent barrier height $\Phi_{\text {app }}$, which is defined as [17]

$$
\Phi_{\text {app }}=\frac{\hbar^{2}}{8 m_{\mathrm{e}}}\left(\frac{\mathrm{d} \ln I}{\mathrm{~d} s}\right)^{2} .
$$

Here, $\mathrm{d} s$ is the change of the tip-sample separation and $m_{\mathrm{e}}$ the electron mass. The apparent barrier height is often used as an estimate for the sample work function. However, its absolute value is connected to the work functions of both tip and sample. The spatially resolved $\Phi_{\text {app }}(x, y)$ map can be seen in Section 7.1, Fig. 7.12(b), with a mean value of $(1.05 \pm 0.17) \mathrm{eV}$. After performing the normalization, the $\mathrm{d} I / \mathrm{d} V(V, x)$ data reveals that the $-0.6 \mathrm{eV}$ peak is strongest at the protrusions of the reconstruction, as seen in Fig. 7.5 (c) for the cross section along the marked direction. However, for this data set, the tunneling current has dropped below $1 \mathrm{pA}$ in the vicinity of the Fermi energy, which could be mistaken for a non-metallic surface. To resolve the energy region around $E_{\mathrm{F}}$, another spectroscopy was performed, which was acquired at a smaller bias voltage set point of $0.2 \mathrm{~V}$. The spatially averaged $\mathrm{d} I / \mathrm{d} V(V)$-curve can be seen in Fig. 7.5 (d) and the full data set in Section 7.1, Fig. 7.13. A finite conductance at $E_{\mathrm{F}}$ is clearly present. Moreover, the differential conductance has a parabolic shape with a minimum shifted slightly toward negative bias voltages with an additional $\mathrm{d} I / \mathrm{d} V(V)$ feature at $0.1 \mathrm{eV}$. The derived apparent barrier height is $\Phi_{\text {app }}=(2.99 \pm 0.27) \mathrm{eV}$. 
7. Surface resonance of the $(2 \times 1)$ reconstructed lanthanum hexaboride (001)-cleavage plane:

A combined STM and DFT study

\section{Surface simulations}

Our experimental findings of a $(2 \times 1)$ reconstructed surface together with previous theoretical predictions [74] point toward a lanthanum terminated $(2 \times 1)$ surface reconstruction. Based on DFT, we performed an in-depth analysis of the electronic surface structure of such a (001) surface of $\mathrm{LaB}_{6}$ with linear chains of lanthanum atoms at the surface, which are separated by void lines. This termination makes the surface formally charge neutral. Details about the DFT simulations, including the unit cell setup as well as technical parameters, are given in Section 7.1. Therein, we also present the relaxed surface structure.

The angular momentum projected density of states (DOS) obtained from our DFT surface calculation is shown in Fig. 7.6. For comparison, we show the orbital-projected DOS of a bulk simulation of $\mathrm{LaB}_{6}$ which is in good agreement with previous DFT results $[97,34]$. In the data of the surface slab we find a characteristic peak $-0.2 \mathrm{eV}$ below the Fermi level, which is not present in the bulk data. This peak is a surface feature and is composed largely of states of boron and lanthanum atoms closest to the surface. The peak is made of boron dangling bonds sticking out of the surface, which bind to the La $d_{x y}$ orbitals lying in the surface plane. The lobes of the $d_{x y}$ orbital point towards the four adjacent boron dangling bonds. While the contributions of the $\mathrm{La} d_{3 z^{2}-r^{2}}$ and $\mathrm{La} d_{x^{2}-y^{2}}$ orbitals are rather small within the peak, they are dominant in the energy intervals adjacent to the peak. The projection of the DOS onto the $d$ orbitals of the La surface atoms is depicted in Fig. 7.7. For additional DOS projections, see Section 7.1.

To connect our DFT simulations more closely to our STM/STS measurements, we follow Bardeen's tunneling theory [8] together with the arguments of Tersoff and Hamann $[94,95]$, which relates the tunneling current for energies close to the Fermi level to the LDOS at the tip apex, integrated from the chemical potential of the probe to that of the tip.

Hence, to simulate the STM images, we compute the LDOS integrated over suitable energy windows. Rather than evaluating the LDOS at realistic tip positions, we choose a shorter distance of $4 \AA$ above the plane of surface La ions. This is necessary, since at much larger distances the exponential decay of the LDOS leads to values that are too small to be resolved in our calculations. Furthermore, 

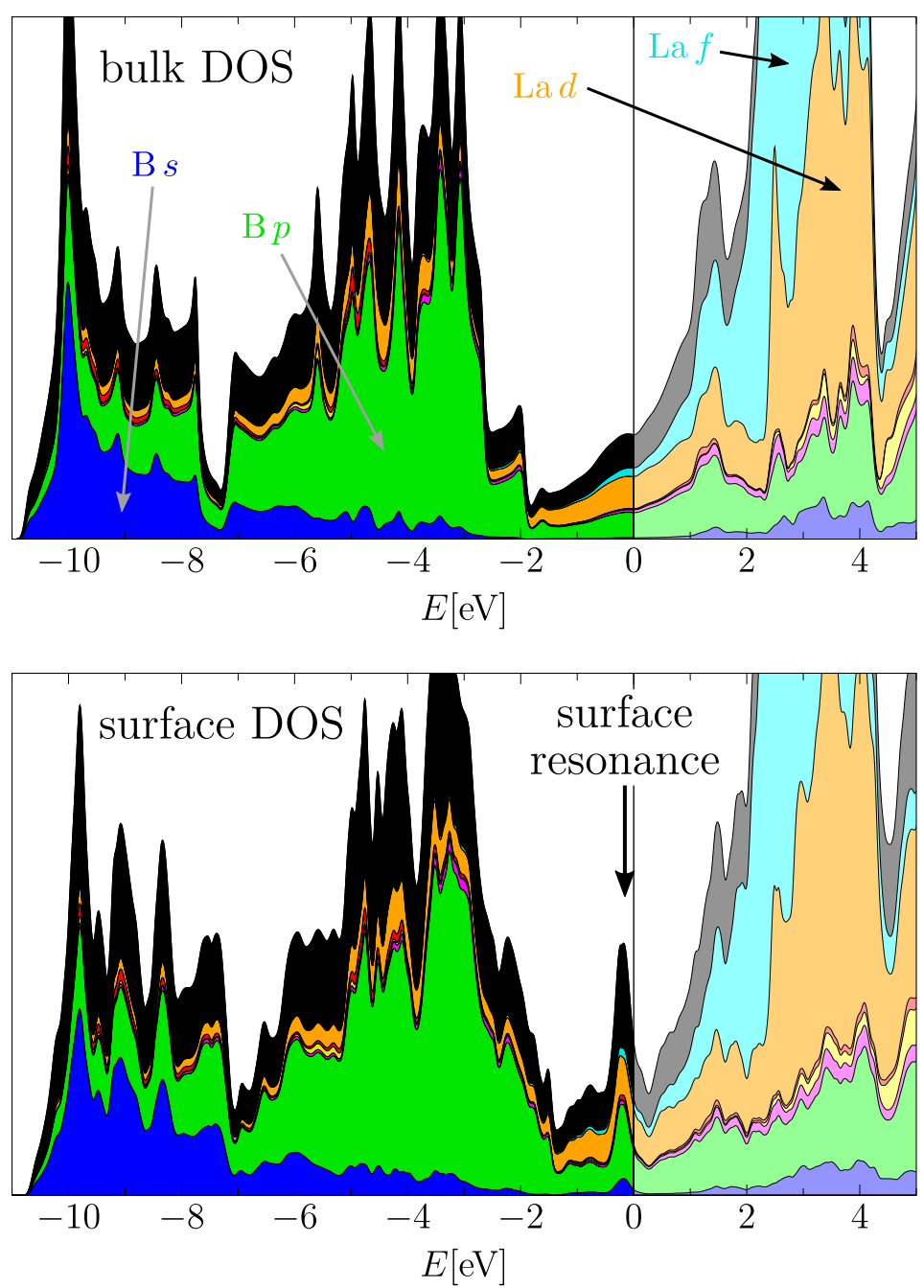

Figure 7.6: Bulk DOS (top) and surface DOS (bottom) in arb. units projected onto atomic orbitals. The individual contributions are stacked. The Fermi energy is set to zero. Color code: Total DOS (not stacked, black), B $s$ (blue), B $p$ (green), B $d$ (magenta), La $s$ (yellow), La $p$ (red), La $d$ (orange), La $f$ (cyan). The characteristic surface peak is indicated by an arrow.

at $4 \AA$ the contrast of the significant features is particularly clear. However, by comparing the images taken at $4 \AA$ with images calculated at larger distances, we ensured that the contrast does not change qualitatively.

Figure 7.8 shows simulated STM images obtained from specific energy windows. At energies below $E_{\mathrm{F}}$, the simulation images are dominated by the boron lone pairs (Fig. 7.8, left and middle graph). The contrast is especially clear in 


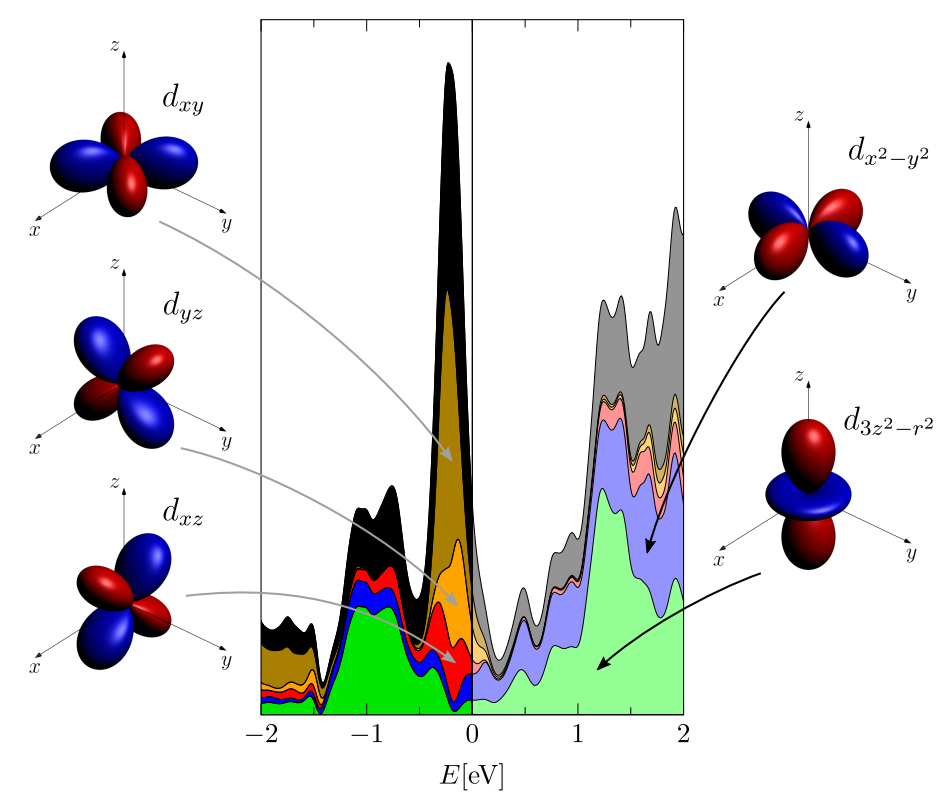

Figure 7.7: DOS projected onto the $d$ orbitals of the La surface atoms, resolved by magnetic quantum number. DOS data in arb. units. The individual contributions are stacked. Color code: total DOS from La surface atoms (black), $d_{x y}$ (gold), $d_{y z}$ (orange), $d_{3 z^{2}-r^{2}}$ (green), $d_{x z}$ (red), $d_{x^{2}-y^{2}}$ (blue).

the energy window covering the characteristic peak in the DOS $0.2 \mathrm{eV}$ below the Fermi level (Fig. 7.8, middle graph). While we observe distinct intensity maxima above the individual boron lone pairs in the energy window of the characteristic peak, we expect them to merge at larger distances, that correspond to realistic tip-surface distances.

The La ions are not visible in the energy windows below $E_{\mathrm{F}}+0.28 \mathrm{eV}$, because the La $d$ orbitals are more localized than the boron lone pairs. In addition, the nodal structures of the La $d$ orbitals lead to a reduced density above the La ion in the range of the characteristic peak. This changes for the energy window above the characteristic peak at $E_{\mathrm{F}}-0.2 \mathrm{eV}$ : in the energy range from $E_{\mathrm{F}}+0.28 \mathrm{eV}$ to $E_{\mathrm{F}}+1.64 \mathrm{eV}$, the La $d_{3 z^{2}-r^{2}}$ orbitals pointing out of the surface contribute largely to the LDOS with the La $f$ orbitals providing an additional share. Hence the intensity is largest on top of the La positions.

To extract the local spectral information, which is provided by STS, we introduced so-called empty atoms above the surface. These atoms do not change the 

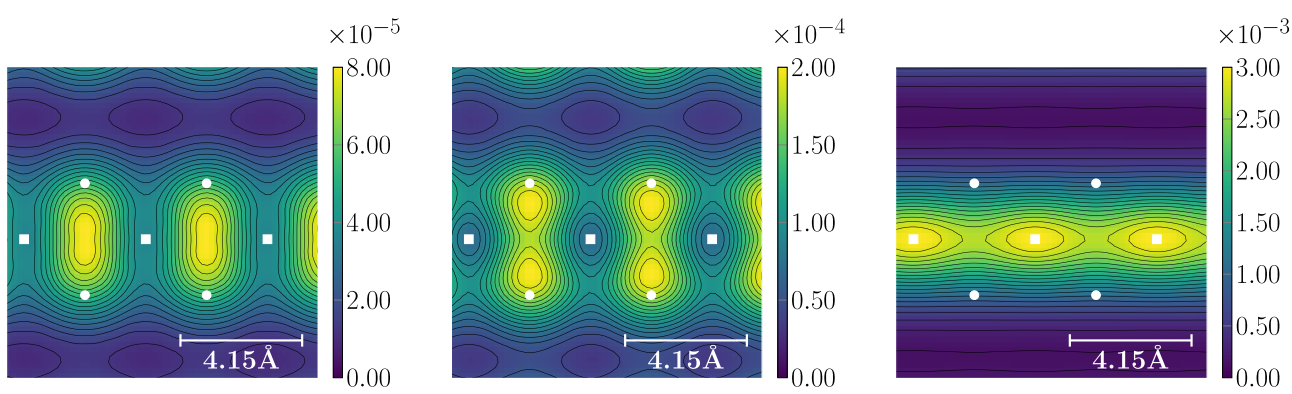

Figure 7.8: Electron density at $4 \AA$ above the surface for electrons from selected energy regions. Color scale in arb. units. Left: $-1.29 \mathrm{eV}$ to $-0.53 \mathrm{eV}$, center: $-0.53 \mathrm{eV}$ to $0.28 \mathrm{eV}$, right: $0.28 \mathrm{eV}$ to $1.64 \mathrm{eV}$. The white squares mark the positions of lanthanum atoms at the surface. White circles indicate the positions of the topmost boron atoms.

physics of the system, but they are a technical trick that allows us to extract the LDOS in the vacuum region. The empty atoms provide local orbitals onto which the wave functions are projected to obtain the projected DOS.

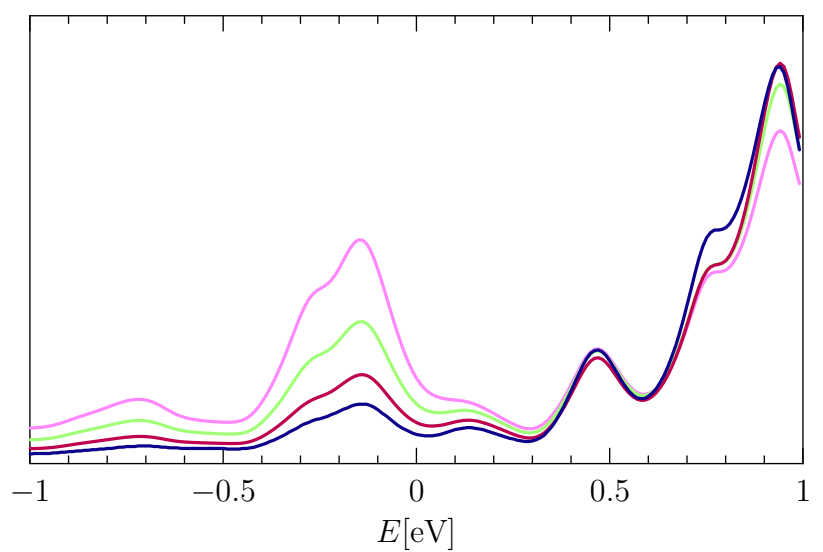

Figure 7.9: DOS of the slab projected onto layers parallel to the slab at distances of $4 \AA$ (pink), $5 \AA$ (green, rescaled $\times 6$ ), $6 \AA$ (red, rescaled $\times 34$ ), and $7 \AA$ (blue, rescaled $\times 200)$ above the surface. The graphs are in arb. units.

The LDOS projected onto layers of empty atoms is shown in Fig. 7.9. The characteristic peak of the boron dangling bonds below the Fermi level is very prominent. In addition, we find a second peak at $E_{\mathrm{F}}+0.47 \mathrm{eV}$. As seen in Fig. 7.7, this peak is due to the $d_{3 z^{2}-r^{2}}$ orbitals pointing out of the surface. At higher energies, in addition to the $d_{3 z^{2}-r^{2}}$ orbitals, the $f$ orbitals start to contribute to the 
7. Surface resonance of the $(2 \times 1)$ reconstructed lanthanum hexaboride (001)-cleavage plane:

A combined STM and DFT study

LDOS.

Above the peak at $E_{\mathrm{F}}+0.47 \mathrm{eV}$, the LDOS rises sharply. The rise of the DOS at higher energies can be attributed to two reasons: First, in this energy window the lanthanum ion has a large DOS due to $d$ and $f$ electrons. Second, wave functions at higher energies extend further out into the vacuum, because of their larger kinetic energy.

By comparing the LDOS at different distances, we find that the intensity of the dangling bond peak decreases faster with increasing distance than the intensity of the LDOS above the Fermi energy. This can be seen in Fig. 7.9.

\section{Discussion}

By investigating the lanthanum hexaboride (001)-cleavage plane in UHV condition, we observed a chainlike $(2 \times 1)$ reconstruction. Our STM experiments show that these chains are mostly labyrinthlike arranged and the number of parallel chains barely exceeds three or four. The most ordered $(2 \times 1)$ reconstruction with a larger number of parallel chains can be found in the vicinity of step edges, where the chains tend to be ordered perpendicularly to the step edge. At a step edge which was rotated by $90^{\circ}$, we observed the respective $(1 \times 2)$ reconstruction. Following these arguments, the observed $(1 \times 2)$ spots in the ULEED are likely caused by a step structure which was rotated by $90^{\circ}$. This supports the assumption that the reconstruction aligns preferentially perpendicularly to step edges.

The atomic surface structure of the $(2 \times 1)$ reconstruction has been proposed as parallel lanthanum chains with a spacing of two lattice constants on top of a full $\mathrm{B}_{6}$ layer [74]. Our DFT slab simulations of this lanthanum chain-terminated surface show that the electronic structure slightly below $E_{\mathrm{F}}$ is governed by a surface resonance. The orbital-projected DOS reveals that the surface resonance is mainly composed of boron $s p$-hybrid dangling bonds pointing out of the surface. In contrast, in the unoccupied states, La $d_{3 z^{2}-r^{2}}$ orbitals are predominant. In comparison to the boron surface states, these orbitals show a slower decay and extend further into the vacuum.

Our simulated STM images provide a translation of these results to the language of CCT images recorded by STM experiments. On the one hand, the dom- 
inant contribution of the $\mathrm{La} d_{3 z^{2}-r^{2}}$ orbitals to the LDOS at $E>E_{\mathrm{F}}$ leads to well-separated chainlike structures above the surface. This is in good agreement with our experimental CCT images, which were taken at positive bias voltages. Hence, at positive bias voltage, the STM addresses mainly the $d_{3 z^{2}-r^{2}}$ orbitals. On the other hand, at $E<E_{\mathrm{F}}$ or negative sample bias voltage, respectively, our theoretical results predict tunneling predominantly from the boron lone pairs to the STM tip. This is difficult to realize experimentally, since we find rather unstable tunneling conditions for negative bias voltages. Hence, imaging the surface at negative bias voltages remains an open task.

The presence of a spectral feature below $E_{\mathrm{F}}$ is, however, verified by our STS measurements. In the tunneling spectra, we find the surface resonance at $-0.6 \mathrm{eV}$ and a parabola-shaped tunneling conductance around $E_{\mathrm{F}}$. Experimentally, an additional feature is found at $0.1 \mathrm{eV}$. These features would coincide with the calculated DOS if $E_{\mathrm{F}}$ was shifted by $+0.4 \mathrm{eV}$ in the calculated DOS. We tested the dependence of the surface resonance position on the density functional by doing additional calculations with the PBE functional, i. e., without Hartree-Fock contribution. The peak position does not change. This can be rationalized from the nature of the state, which is mostly boronlike and has little La $d$ and no La $f$ contribution.

Although the investigated $(2 \times 1)$ structure is far from pristine and exhibits numerous kinks and defects, one can exclude that the surface resonance position depends on the presence of surface defects. Experimentally, we do not observe a shift of the surface resonance regardless of the local surface morphology, as seen in Fig. 7.12. Furthermore, the main features of the experimental tunneling spectra can be clearly identified in our DFT results on the defect-free surface.

A small shift in the chemical potential, which may explain the relative shift of $0.4 \mathrm{eV}$ between experimental and theoretical spectra, could originate from a nonstoichiometric crystal. During crystal growth, preferential evaporation of boron atoms leaves the formed crystal slightly boron deficient [56]. Thus, the substance ratio $\mathrm{La} / \mathrm{B}$ can be somewhat larger than the stoichiometric 1/6. For the present sample, the extra lanthanum atoms would lead to a surplus of charge carriers, shifting the Fermi level to higher energies.

The measured apparent barrier height is rather small with values in the range 
7. Surface resonance of the $(2 \times 1)$ reconstructed lanthanum hexaboride (001)-cleavage plane:

A combined STM and DFT study

from $1 \mathrm{eV}$ to $3 \mathrm{eV}$. This is in agreement with the low work function of about $2.5 \mathrm{eV}$ obtained in former studies [96].

Moreover, the knowledge that the $(2 \times 1)$ reconstruction consists of lanthanum chains can be used to assign the termination of atomic steps. A step between two $(2 \times 1)$ reconstructed areas should be of integer multiple height of the bulk lattice constant, since the termination is equal on each side. Such steps have been observed. For the area shown in the left of Fig. 7.11, the step height is smaller than one bulk lattice constant. Here, we suggest a boron-rich termination with an almost vacant lanthanum layer at the surface. However, we would like to point out that this is one of the very few steps of that height we have found so far.

The surface morphology observed in this study is different to that of previous UHV STM investigations of $\mathrm{LaB}_{6}[58,59]$. In these studies, a $(1 \times 1)$ reconstructed (001) surface was found, which is stated to be lanthanum terminated and about $10 \%$ of the surface's lanthanum sites are vacant. However, it should be noted that the samples used in these studies are prepared by polishing and heating instead of cleavage as in our study, which likely leads to the different surface structures observed. Our structural results are more comparable to the findings on the cleaved surface of $\mathrm{SmB}_{6}$. For cleaved $\mathrm{SmB}_{6}$, surface step heights are only of integer multiples of the bulk lattice constant if the terminations on both sides are of the same kind [45], as observed in this study as well. Cleavage of $\mathrm{SmB}_{6}$ can take place by breaking either the bonds within the boron octahedra or the bonds between the octahedra. Hence, it is lacking a natural cleavage plane [86, 71, 47], and atomically ordered areas occur rather infrequently $[45,36]$. Since $\mathrm{LaB}_{6}$ has the same crystal structure, no natural cleavage plane is present. This might be the reason for the mostly disordered surfaces we have observed so far. Similar to our observations, LEED investigations on cleaved $\mathrm{SmB}_{6}$ samples show only $(2 \times 1)$ spots on certain surface areas and the diffraction pattern is governed by $(1 \times 1)$ spots [66]. Therefore, the $(1 \times 1)$ spots have been associated with the bulk periodicity due to the finite penetration depth of the electrons. The same arguments should be applied for our sample system to explain the dominant $(1 \times 1)$ spots. 
7.1. Original publication

\section{Conclusion}

In a combined study of STM/STS, LEED, and DFT, we have investigated the (001)-cleavage plane of $\mathrm{LaB}_{6}$. Atomically ordered areas are labyrinthlike $(2 \times$ 1) reconstructed. These chains can be understood as parallel rows of lanthanum atoms on top of $\mathrm{a}_{6}$ layer, with a surface resonance below $E_{\mathrm{F}}$. Electronically, this resonance is mainly composed of $\mathrm{B} s p$-hybrid orbitals and La $d_{x y}$ states originating from the surface atoms. Lanthanum hexaboride is the electronically most simple candidate of the $R \mathrm{~B}_{6}$ family. However, understanding the electronic surface structure is not straightforward and differs severely from that of the bulk. Moreover, the $\mathrm{LaB}_{6}$ surface morphology is more complex than previously discussed. Since all $R \mathrm{~B}_{6}$ have the same crystal structure, our findings could help to further understand the general surface physics of hexaborides.

We acknowledge fruitful discussions with S. R. Manmana. The orientation of the single crystals via gamma ray diffraction were carried out in the group of G. Eckold by F. Ziegler and P. Kirscht at the Institut für Physikalische Chemie, University of Göttingen. We gratefully acknowledge financial support by the DFG Grants No. WE1889/10-1, No. BL539/10-1, and No. PR298/19-1. Furthermore, we acknowledge financial support by the SFB 1073 through Projects No. B03, No. C03, and No. C04. The work at the University of Warwick was supported by the EPSRC, UK, through Grant No. EP/M028771/1. The images in Fig. 7.1, Fig. 7.14 and Fig. 7.15 were created with VESTA [51].

F. Sohn and P. Buchsteiner contributed equally to this work. 
7. Surface resonance of the $(2 \times 1)$ reconstructed lanthanum hexaboride (001)-cleavage plane:

A combined STM and DFT study

\section{Appendix}

\section{Supporting experimental data}

Figure 7.10 (a) shows a typical large scale topography of the cleaved $\mathrm{LaB}_{6}(001)$ surface as observed with AFM. Flat terraces within the AFM resolution with areas of several hundred (nm $)^{2}$ up to $(\mu \mathrm{m})^{2}$ in size can be easily found. These areas are connected by steps of integer multiples of the bulk lattice constant of $4.15 \AA[83,27,10,80,26,33,74]$. The steps in Fig. 7.10 (a) are all of a height of one lattice constant. However, on a smaller length scale probed by STM, up to nanometer-sized protrusions with no pristine long-range order are commonly observed, Fig. 7.10 (b). This results are similar to the STM findings on cleaved $\mathrm{SmB}_{6}[45]$.
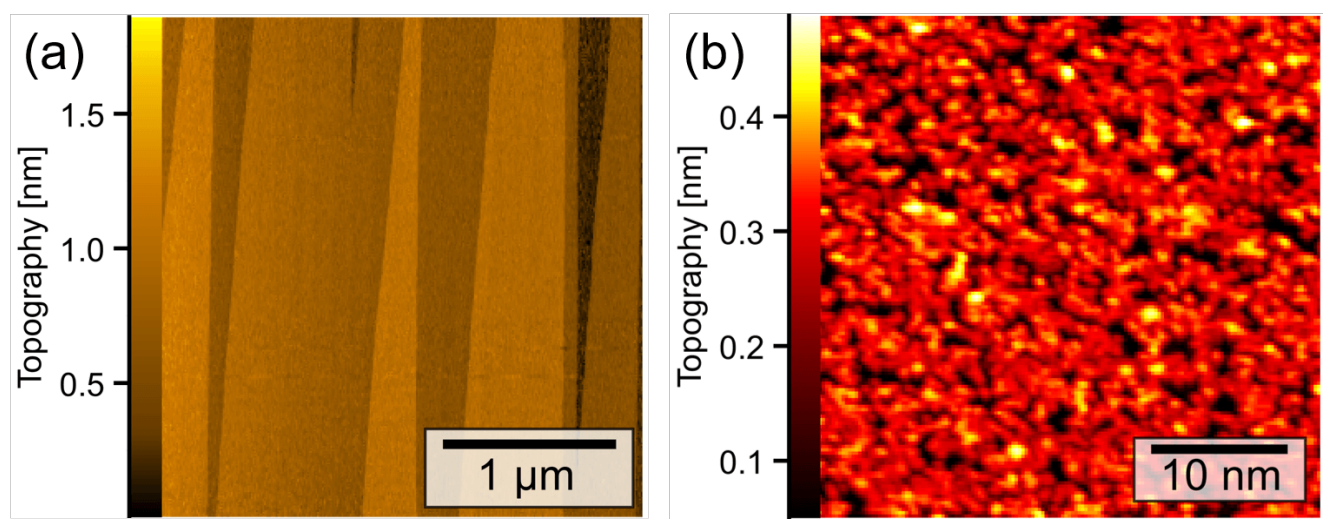

Figure 7.10: (a) (001) surface of cleaved $\mathrm{LaB}_{6}$ as observed with AFM under ambient conditions. (b) CCT of a disordered surface area taken at $1 \mathrm{~V} / 0.1 \mathrm{nA}$.

As described in the discussion above, $\mathrm{LaB}_{6}$ is lacking a natural cleavage plane. If the cleavage leaves the boron cage intact, one dangling bond per octahedron is exposed. The situation changes dramatically, if the cleavage proceeds through the octahedron. The disrupted boron cages expose numerous dangling bonds, which might increase the probability of attaching adsorbates like hydrogen [75]. Another reason for a frequently observed disordered morphology might be that the crystals were cleaved at room temperature. For $\mathrm{SmB}_{6}$, it is reported, that atomically flat surfaces could not be obtained by cleavage at room temperature, but only at about $20 \mathrm{~K}$ [67]. Our present setup does not allow the cleavage at cryogenic temperature. 
An additional experimental reason for the widely disordered appearance might be the tip itself. As seen in Fig. 7.2, even when atomic chains can be resolved, they are mostly labyrinthlike arranged. If the same area would have been scanned with a slightly blunt tip, the topography certainly would appear rather disordered, too.
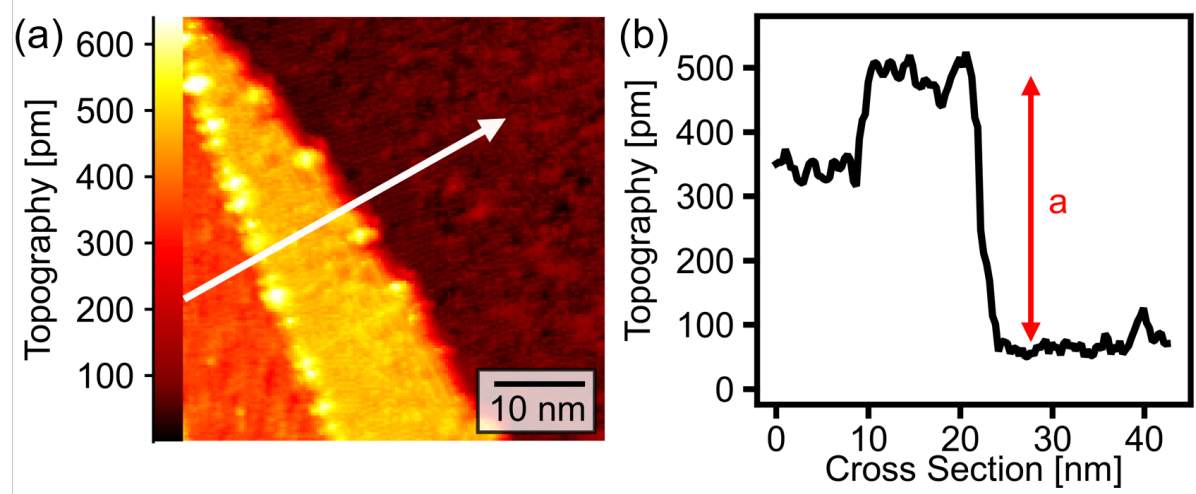

Figure 7.11: (a) CCT image taken at $0.8 \mathrm{~V} / 0.1 \mathrm{nA}$ of two steps, one of about $1.3 \AA$ height and one of a bulk lattice constant height, as shown in the height profile in (b).

We have found a step with a height of only a fraction of the bulk lattice constant, which is shown in Fig. 7.11. On the right-hand side, the already discussed (2 $\times 1$ ) labyrinthlike structure is observed and the step height is again one bulk lattice constant. Interestingly, the step on the left hand side is only about $1.3 \AA$ high. We interpret this finding as a different surface termination, as described in the discussion above.

Figure 7.12 shows the spectroscopy of Fig. 7.5 again, but here including the measured apparent barrier height and another $\mathrm{d} I / \mathrm{d} V(V, x)$ cross section. The mean value of the $\Phi_{\text {app }}$ map is $(1.05 \pm 0.17) \mathrm{eV}$. However, the specific value of the apparent barrier height is correlated with the topography. At the atomic protrusion, blue-marked position in Fig. 7.5 (a), its value is about $1.1 \mathrm{eV}$ and at the green-marked position $1.0 \mathrm{eV}$. For the various surface areas with no clear atomic rows included, the value of $\Phi_{\text {app }}(x, y)$ varies for each tip position.

Figure 7.13 shows the second spectroscopy data set, including the CCT, the $\Phi_{\text {app }}(x, y)$ map, and a $\mathrm{d} I / \mathrm{d} V(V, x)$ cross section after topography normalization. In this bias voltage range, the $\mathrm{d} I / \mathrm{d} V(V)$ curves have their largest values on top 
7. Surface resonance of the $(2 \times 1)$ reconstructed lanthanum hexaboride (001)-cleavage plane:
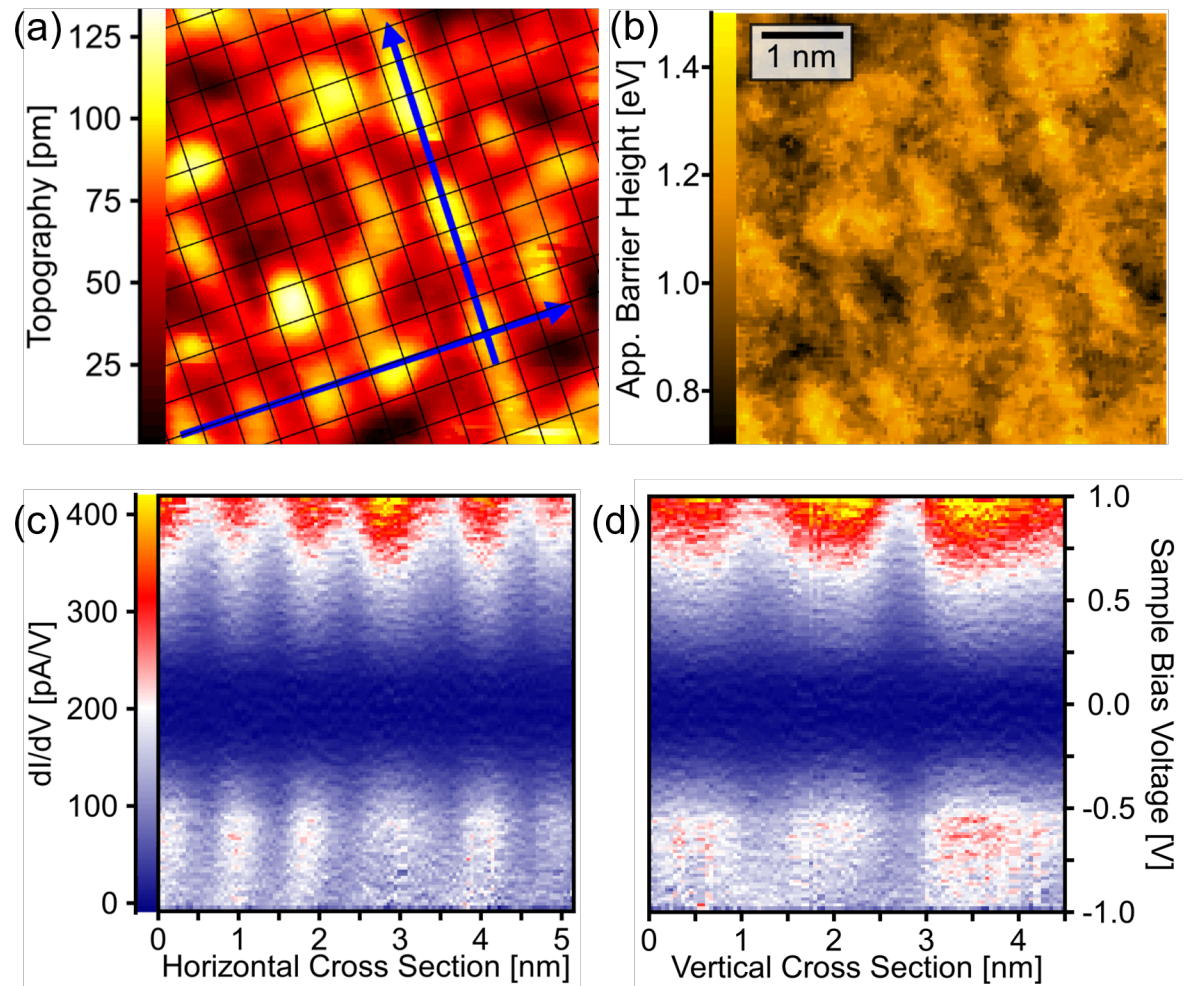

Figure 7.12: (a) The CCT of Fig. 7.5 is shown again, this time with an overlay of the atomic lattice grid for scale. The blue arrows mark the lines along which the horizontal and vertical cross sections are taken. The resulting color coded $\mathrm{d} I / \mathrm{d} V(V, x)$ maps can be seen in (c) and (d). In (b) the spatially resolved apparent barrier height is depicted.

of the reconstruction's protrusion.

\section{Additional detail on the DFT simulations}

A scheme of a simulated slab and its $(2 \times 1)$ surface unit cell is depicted in Fig. 7.14.

The lattice constant was kept fixed at a value of $a=4.15 \AA$ found in former experimental and theoretical studies [83, 27, 10, 80, 26, 33, 74]. The vacuum thickness between the slabs was set to $16.6 \AA$. To avoid artifacts and to have a sufficiently large spatial separation of the slab's top and bottom surfaces and surface states, respectively, a minimum slab thickness of three layers of boron octahedra 


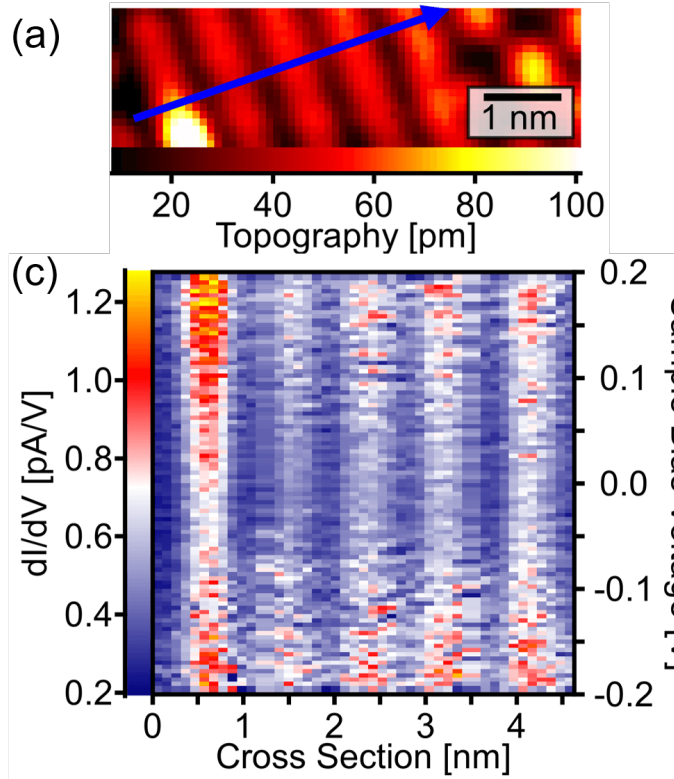

(b)

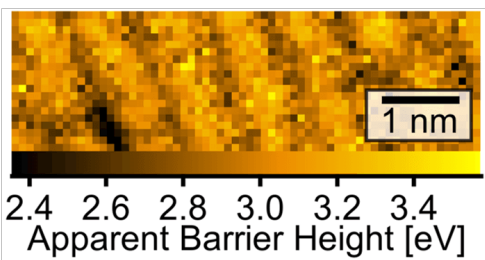

(d)

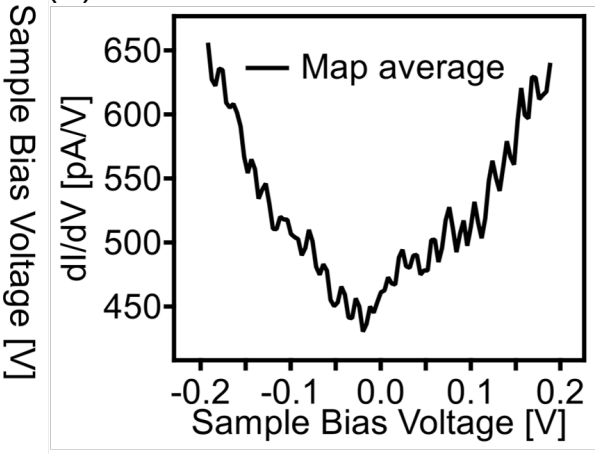

Figure 7.13: (a) CCT taken at $0.2 \mathrm{~V} / 0.1 \mathrm{nA}$. The apparent barrier height is shown in (b). The color coded $\mathrm{d} I / \mathrm{d} V(V, x)$ cross section taken along the blue arrow within (a) is depicted in (c). (d) shows the map averaged $\mathrm{d} I / \mathrm{d} V(V)$ curve of (a).

is required. For our simulations, we used surface supercells with three, five, and seven layers of boron octahedra. This was done to ensure convergence of our DFT data with respect to the slab thickness. In this paper, we present results from our simulations with the seven-layer slab unit cell only. However, all slab thicknesses lead to similar results in atomic structure as well as electronic structure.

Moreover, we used a total amount of $8 \times 4 \times 1 \mathrm{k}$-points. The plane-wave cut-offs were set to $50 \mathrm{Ry}$ for the wave functions and to $100 \mathrm{Ry}$ for the charge density. Furthermore, we used $(2,2,2,1)$ projector functions for the $\mathrm{La}(s, p, d, f)$ states and $(2,2,1)$ projector functions for the $\mathrm{B}(s, p, d)$ states, respectively. The hybrid functional mixing factor was fixed at 0.15 for both atomic species.

In Fig. 7.15, we show the side view of the relaxed surface structure, which exhibits a tilt of the surface octahedra towards the filled chain of La ions. This can be rationalized by the attraction between positive La and negative $\mathrm{B}$ ions: The terminating boron atoms have lone-pairs pointing out of the surface, which hybridize with the La $d_{x y}$ orbitals lying in the surface plane (cf. Fig. 7.7 and discussion in Section 7.1). 

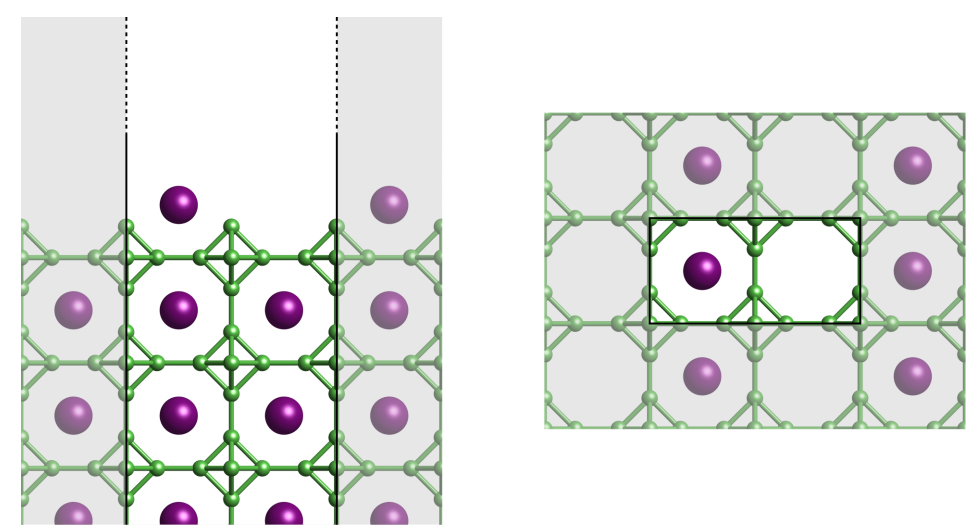

Figure 7.14: Side and top view of the (001) surface of $\mathrm{LaB}_{6}$. The slab unit cell is highlighted by a white background and marked with the black lines. On the surface only every second La row is filled.

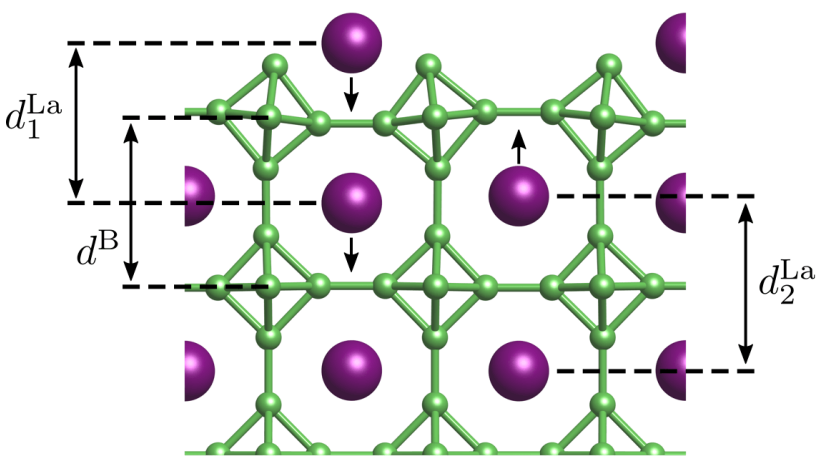

Figure 7.15: Structure relaxation at the slab surface. $d^{\mathrm{La}}$ are distances between La atoms of the topmost layers as indicated. $d^{\mathrm{B}}$ is the distance between the centers of mass of the two octahedra. The small black arrows indicate the direction of the relaxation movement compared to the bulk structure.

Furthermore, the surface relaxation leads to changes in the distance between two layers which can be quantified via the relative change to the bulk lattice constant $a_{\text {bulk }}$, i.e.,

$$
\Delta=\frac{d-a_{\text {bulk }}}{a_{\text {bulk }}}
$$

The surface La ion relaxes inward by $\Delta_{1}^{\mathrm{La}}=-4.3 \%$ relative to the bulk, while the subsurface La ion below the empty La surface site relaxes outward by $\Delta_{2}^{\mathrm{La}}=$ $+4.7 \%$. The boron octahedra exhibit a small outward relaxation of $\Delta^{\mathrm{B}}=+1.4 \%$. 
A comparison of these quantities between the data from the seven- $\mathrm{B}_{6}$-layer slab to the data of the five- $\mathrm{B}_{6}$-layer slab shows only minor differences which amount up to $0.007 \AA$ in absolute values, which is negligible in the context of our calculations. Hence, we expect no significant difference in the DFT results for even larger slab thicknesses. Previous data published by [74] are $\Delta_{1}^{\mathrm{La}}=-6.2 \%, \Delta_{2}^{\mathrm{La}}=+3.5 \%$, and $\Delta^{\mathrm{B}}=+0.4 \%$, respectively. In comparison, the distances in our data are consistently a bit longer.

In Fig. 7.16, we provide two additional projections of the DOS: A projection onto the orbitals of the topmost atoms of either species, and a projection onto the boron dangling bonds. 
7. Surface resonance of the $(2 \times 1)$ reconstructed lanthanum hexaboride (001)-cleavage plane:
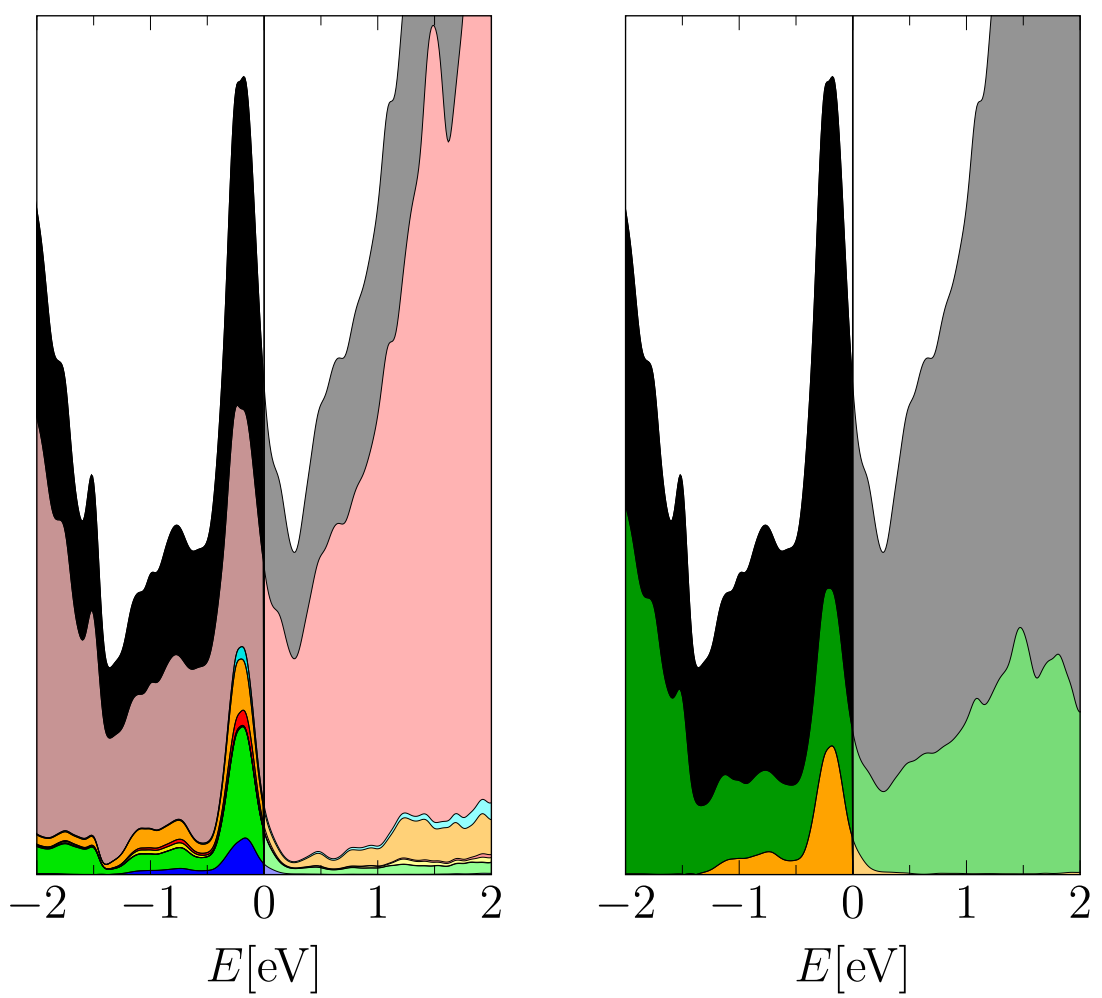

Figure 7.16: Projections of the DOS onto orbitals of the surface layer atoms. DOS data in arb. units. The individual contributions are stacked. Left: DOS projected onto the topmost boron and lanthanum atoms. The color code is identical to the color code in Fig. 7.6. The bulk contribution is stacked on top (reddish brown). Right: DOS projected onto boron orbitals. The dangling bonds of the boron surface atoms (orange) dominate the peak close to the Fermi level. The contribution of all other boron orbitals of the slab (dark green) is shown for comparison. 


\section{Chapter 8}

\section{Surface analysis of the $\operatorname{PrB}_{6}$ (001) cleavage plane by scanning tunneling microscopy and spectroscopy}

The previous work focussed on the $\mathrm{LaB}_{6}$ (001)-cleavage plane. It showed that there is a significant difference between bulk and surface electronic structure. A major goal of this thesis was furthermore the investigation of a $4 f$ system, as described in Chapter 1. In the upcoming Section 8.1, we aimed to investigate the influence of the $4 f$ electrons on the $\mathrm{PrB}_{6}$ (001)-cleavage plane using STM and STS.

We chose $\operatorname{PrB}_{6}$ for several reasons. Firstly, it shows a complex sequence of magnetic structures at low temperatures [49]. At $6.9 \mathrm{~K} \mathrm{PrB}_{6}$ orders from a paragmagnetic phase to an incommensurate antiferromagnetic phase. Between $6.9 \mathrm{~K}$ and $4.2 \mathrm{~K}$ the ordering is both incommensurate and commensurate. At $1.74 \mathrm{~K}$ only the commensurate phase prevails. Interestingly, already at temperatures below $\approx 20 \mathrm{~K}$ short-range ordering occurs [76]. Subsequent experiments showed, that this spin-glass transition not occurs when using annealed crystals [3]. Since the annealing eliminates boron defects it was concluded that the short-range ordering accumulates in the vicinity of boron defects. In our setup, we investigate 
8. Surface analysis of the $\operatorname{PrB}_{6}$ (001) cleavage plane by scanning tunneling microscopy and spectroscopy

the (001)-cleavage plane at $8 \mathrm{~K}$. Consequently, due to the cleavage boron defects are produced and the temperature of $8 \mathrm{~K}$ lies in the exciting range of the spin-glass phase.

Secondly, before starting the STM experiments it had to be ensured whether the $4 f$ electrons are potentially addressable within the typical bias voltage range of $\pm 2 \mathrm{~V}$. Photoemission experiments reveal spectral intensity at about $-1 \mathrm{eV}$ binding energy stemming from the $4 f$ electrons $[61,60]$, an energy range which is conveniently accessable with STM.

Thirdly, hexaboride surfaces, as seen for $\mathrm{LaB}_{6}$ and $\mathrm{SmB}_{6}$, can possess a versatile surface structure. We expect different surface reconstructions on $\operatorname{PrB}_{6}$ as well with an individual influence of the $4 f$ electrons on each reconstruction. Consequently, with one single cleave several $4 f$ systems might be present.

Fourthly, our collaborators in Warwick provided us with a large $\operatorname{PrB}_{6}$ single crystal of excellent quality, which was from a practical point of view perfectly suited for our experiments, as seen in Chapter 4.

Fifthly, up to the following investigation, only metallic $\mathrm{LaB}_{6}[12]$, Kondo lattice $\mathrm{CeB}_{6}$ [22], Kondo insulating $\mathrm{SmB}_{6}$ [67] and ferromagnetic $\mathrm{EuB}_{6}$ [65] have been investigated by STM. Here, we are providing the first results of an antiferromagnetic hexaboride.

The following Section 8.1 has been published as article in Phys. Rev. B 102, 205403 (2020), DOI: 10.1103/PhysRevB.102.205403. The manuscript was drafted by P. Buchsteiner and M. Wenderoth. STM experiments were carried out by L. Harmsen within her master thesis [30] and by P. Buchsteiner. STM data interpretation and analysis were done by L. Harmsen, P. Buchsteiner and M. Wenderoth. Single crystal growth was performed by M. Ciomaga Hatnean and G. Balakrishnan.

Minor adaptions were made for the reprint of the article, for example order of references, enumeration of figures and sections, changes of font and font size. 


\subsection{Original publication}

Scanning tunneling microscopy and spectroscopy were performed on the (001) cleavage plane of praseodymium hexaboride $\left(\operatorname{PrB}_{6}\right)$. We found three different ordered morphologies, namely, a chainlike $(2 \times 1)$ reconstruction and two uniform terminations. The chainlike $(2 \times 1)$ reconstruction is rationalized as parallel Pr rows on top of a complete $B_{6}$ network. The two uniform terminations are identified as complete $\operatorname{Pr}$ or $\mathbf{B}_{6}$ layers. Although the uniform terminations could be expected to be simply $(1 \times 1)$ reconstructed, one of them shows a rather stripelike atomic corrugation for close tip-sample distances. All morphologies share two spectral features at -0.2 and $+0.2 \mathrm{eV}$ around $E_{\mathrm{F}}$. In addition, one uniform termination shows an additional peak in the differential conductance at $-0.7 \mathrm{eV}$. Similarly, the chainlike $(2 \times 1)$ reconstruction reveals a feature in the differential conductance at $-1.1 \mathrm{eV}$ when moving the tip closer to the surface. The distance dependency points towards rather localized electronic states, which we tentatively attribute to a $4 f$-related feature.

P. Buchsteiner, L. Harmsen, M. Ciomaga Hatnean, G. Balakrishnan, M. Wenderoth

\section{Introduction}

For several decades, the surface properties of rare-earth hexaborides $\left(R \mathrm{~B}_{6}\right)$ have been of steady interest for both technological application and fundamental science. In particular, lanthanum hexaboride $\left(\mathrm{LaB}_{6}\right)$ and samarium hexaboride $\left(\mathrm{SmB}_{6}\right)$ are in the focus of today's research. $\mathrm{LaB}_{6}$ has an unusually low work function and is therefore commonly used as an electron emitter [96]. $\mathrm{SmB}_{6}$ is a Kondo insulator $[37,82]$ with proposed topologically insulating surface states [21]. This variety of physical properties in $R \mathrm{~B}_{6}$ can be traced back to their $4 f$ electron occupancy. For instance, La has no occupied $4 f$ electron, whereas Sm has a $4 f^{5}$ configuration. In order to further investigate the influence of $4 f$ electrons on the surface physics, we add praseodymium hexaboride $\left(\operatorname{PrB}_{6}\right)$ as the material of interest in this paper. 
8. Surface analysis of the $\operatorname{PrB}_{6}$ (001) cleavage plane by scanning tunneling microscopy and spectroscopy

From a technological perspective, a thorough understanding of the $\operatorname{PrB}_{6}$ surface is desired for the growing interest in hexaboride nanocrystals [91, 102, 104, 16]. While nanoparticulate LaB6 is already used as solar control filters [2], nanoparticulate hexaborides are, in general, promising candidates for heat shieldings [16]. Of course, for nanocrystals, the surface-to-bulk ratio is noticeably increased and a thorough understanding of the $4 f$ surface physics of hexaborides is inevitable for their practical utilization.

Pr has a $4 f^{3}$ configuration and, as other hexaborides with a partially filled $f$ shell, $\operatorname{PrB}_{6}$ has an antiferromagnetic ground state [24]. The antiferromagnetic phase transitions occur below $T_{\mathrm{N}} \approx 6.9 \mathrm{~K}$ [49]. However, already at temperatures below $\approx 20 \mathrm{~K}$, short-range ordering occurs [76], which is pinned in the vicinity of boron vacancies [3]. At $10 \mathrm{~K}$, which is well above $T_{\mathrm{N}}$, photoemission spectroscopy (PES) has revealed spectral intensity attributed to the occupied $4 f$ electrons at around $1 \mathrm{eV}$ binding energy $[61,60]$. Electronic structure calculations using density functional theory (DFT) of the $\operatorname{PrB}_{6}$ bulk suggested $4 f$ intensity in this energy region as well [78]. Please note that while photoemission experiments typically average over several $(\mu \mathrm{m})^{2}$ of the investigated surface, the atomic structure of hexaboride surfaces might exhibit various morphologies, as, e.g., observed for $\mathrm{LaB}_{6}[58,59,12]$ and $\mathrm{SmB}_{6}[45,86,71,36,67,103,46,64]$. Hence, local probe methods such as scanning tunneling microscopy (STM) might be necessary to address the interplay between the $4 f$ electrons and the underlying atomic corrugation.

The crystal structure of $R \mathrm{~B}_{6}$ is simple cubic, where the rare-earth ion is situated in the cube's center, as seen in Fig. 8.1 (f). The boron atoms are arranged in octahedra, which are connected by a three-dimensional covalent binding network. The stability of the boron network is established via electron donation of the host metal [43], which inevitably creates an ionic character. Thus, at the resulting surface, a uniform rare-earth or $\mathrm{B}_{6}$ termination would be polar. The buildup of a polar surface can be avoided by a simple $(2 \times 1)$ reconstruction. This has been reported for $\mathrm{LaB}_{6}[12,74]$ and $\mathrm{SmB}_{6}[45,67,103,46,64]$, where the rare-earth ions are arranged in parallel chains. 


\section{Methods}

The experiments were carried out in a home-built scanning tunneling microscope at a base pressure of $6 \times 10^{-11}$ mbar. Constant current topographies (CCTs) as well as local spectroscopy were acquired at a temperature of $8 \mathrm{~K}$. The $\operatorname{PrB}_{6} \sin$ gle crystal was grown by the floating zone technique, which is described in more detail in $[6,7]$. The orientation of the single crystal was done by gamma ray diffraction. The crystal was cut into sample dimensions of about $1 \times 4(\mathrm{~mm})^{2}$ and $300 \mu \mathrm{m}$ thickness. The samples were cleaved in situ to expose the (001) surface and were transferred immediately into the STM head at $8 \mathrm{~K}$. Tunneling tips were made from electrochemically etched tungsten wire. The tunneling spectra were recorded using a lock-in technique, if not stated otherwise, and the modulation voltages are denoted by $V_{\text {mod }}$. Similar to recent STM studies on other hexaborides, we find the surface to be rather vulnerable. We attribute the high noise level in the presented data explicitly to the sample system. Spatially resolved spectroscopy was rarely successful. Although CCT maps a contour of the integrated local density of states (LDOS) from the Fermi energy up to the applied bias voltage, multibias CCT can provide an estimation for the variation in the LDOS, especially when the $I(V)$ curves show a steep increase for higher bias voltages. The multibias data sets consist of several CCTs, which were acquired quasisimultaneously. The multibias images were not recorded subsequently, but each scan line was recorded with the respective bias voltages before moving to the next scan line. This approach assures that we can exclude tip modification for the interpretation. Additionally, the signal-to-noise ratio of a CCT image can be improved by a so-called template average, as described in [23]. If the CCT image contains a periodic lattice, the periodicity can be used to calculate an averaged unit cell from a given number of examined unit cells. The resulting template topography represents the averaged unit cell, which is periodically extended.

\section{Results}

Figure 8.1 shows two large-scale topographies of the $\operatorname{PrB}_{6}(001)$ cleavage plane. In Fig. 8.1 (a), a uniform terminated surface is presented, which contains a step 
8. Surface analysis of the $\operatorname{PrB}_{6}$ (001) cleavage plane by scanning tunneling microscopy and spectroscopy

edge. The respective cross sections are examined in Fig. 8.1 (c) and Fig. 8.1 (d). The adjacent surfaces of the step edge are partly covered with disordered protrusions and atomically flat areas extend only over a few $(\mathrm{nm})^{2}$. Similar disordered morphologies have been observed on cleaved $\mathrm{LaB}_{6}$ [12] and $\mathrm{SmB}_{6}$ [45]. Here, the disordered morphology is examined in the Appendix; see Fig. 8.5. In Fig. 8.1 (b), the chainlike $(2 \times 1)$ reconstruction is shown and a cross section examining the spacing between the chains is shown in Fig. 8.1 (e).
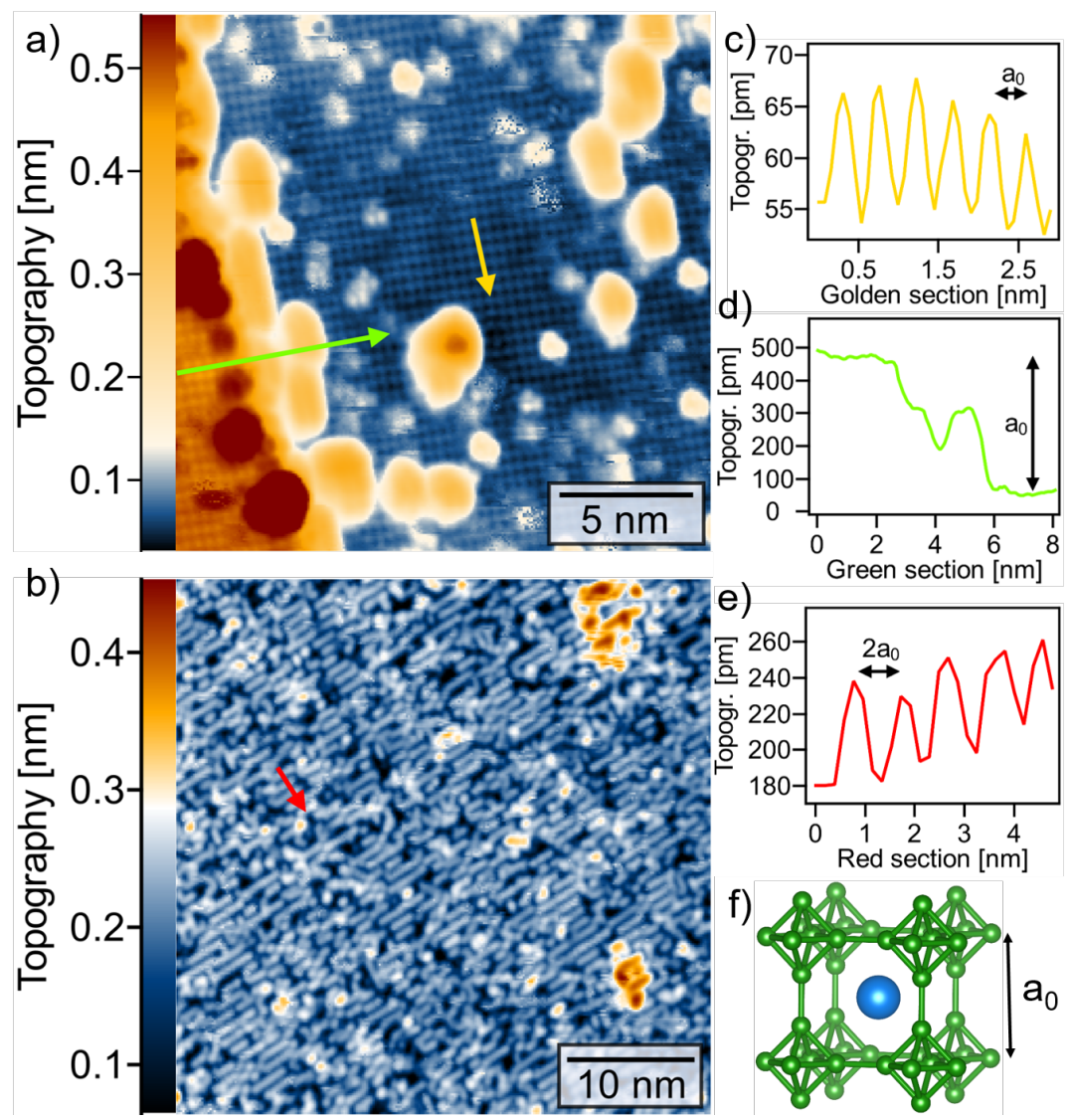

Figure 8.1: (a) CCT of a surface step taken at $0.4 \mathrm{~V} / 0.1 \mathrm{nA}$. (b) CCT of a chainlike $(2 \times 1)$ reconstructed surface taken at $0.4 \mathrm{~V} / 0.1 \mathrm{nA}$. (c) Cross section taken along the golden arrow as indicated in (a). (d) Cross section as indicated by the green arrow in (a). (e) Cross section taken along the red arrow as indicated in (b). (f) Crystal structure of $\operatorname{PrB}_{6}$. The bulk lattice constant of $\operatorname{PrB}_{6}$ is about $a_{0}=4.1 \AA$ [49]. The image in (f) was created with VESTA [51].

The step in Fig. 8.1 (a) is of one bulk lattice constant $a_{0}$ height, which im- 
plies that both adjacent terminations are equal. However, determining the surface terminations of $\mathrm{PrB}_{6}$ is not straightforward. Figure 8.2 displays three different ordered surface morphologies found on the cleavage surface, namely, a chainlike $(2 \times 1)$ reconstruction as well as two uniform terminated structures. We applied multibias CCT as well as local spectroscopy to analyze the electronic properties, which are used to assign the surface terminations.
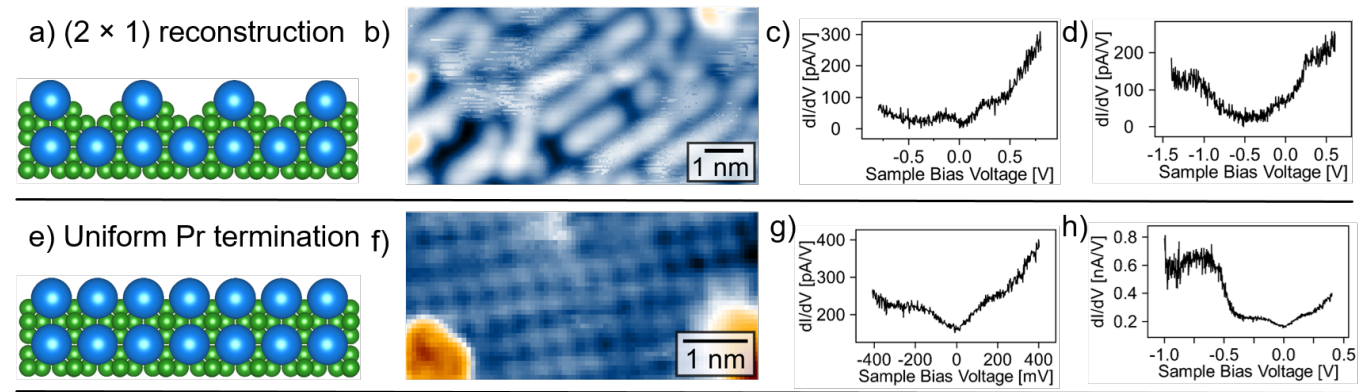

i) Uniform $B_{6}$ termination j)
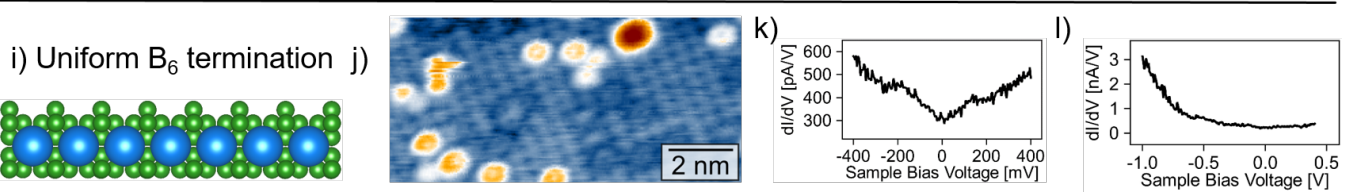

Figure 8.2: Overview of the encountered ordered surface morphologies. (a) Schematic sideview of the chainlike $(2 \times 1)$ reconstruction. (b) CCT of the chainlike $(2 \times 1)$ reconstruction taken at $0.4 \mathrm{~V} / 0.1 \mathrm{nA}$. (c) Tunneling spectroscopy taken on top of an atomic chain, $0.8 \mathrm{~V} / 0.1 \mathrm{nA}$, and $V_{\text {mod }}=5 \mathrm{mV}$. (d) Tunneling spectroscopy taken on top of an atomic chain, $0.6 \mathrm{~V} / 0.1 \mathrm{nA}$, and $V_{\text {mod }}=5 \mathrm{mV}$. (e) Scheme of termination 2, which is assigned as uniform Pr termination. (f) CCT of the uniform Pr termination taken at $1 \mathrm{~V} / 0.1 \mathrm{nA}$. (g) $d I / d V$ curve taken on top of an atomic protrusion, $0.4 \mathrm{~V} / 0.1 \mathrm{nA}$, and $V_{\text {mod }}=10 \mathrm{mV}$. (h) $d I / d V$ curve taken on top of an atomic protrusion, $0.4 \mathrm{~V} / 0.1 \mathrm{nA}$, and $V_{\mathrm{mod}}=10 \mathrm{mV}$. (i) Scheme of termination 3, which is assigned as uniform $\mathrm{B}_{6}$ termination. (j) CCT of the uniform $\mathrm{B}_{6}$ termination taken at $-0.1 \mathrm{~V} / 0.1 \mathrm{nA}$. (k) The $d I / d V(V)$ curve was taken on top of an atomic protrusion at $0.4 \mathrm{~V} / 0.1 \mathrm{nA}$, and $V_{\text {mod }}=10 \mathrm{mV}$. Furthermore, this spectrum was averaged over 5 spectra. (1) $d I / d V$ curve taken on top of an atomic protrusion, $0.4 \mathrm{~V} / 0.1 \mathrm{nA}$, and $V_{\text {mod }}=10 \mathrm{mV}$. The images in (a), (e), and (i) were created with VESTA [51].

Figure 8.2 (a) shows a schematic sideview of the chainlike $(2 \times 1)$ reconstruction, which is called termination 1 in the following. Ordered atomic rows as observed by CCT [see Fig. 8.2 (b)] are only of a few $\mathrm{nm}$ in length and can have a predominant alignment over several-hundred $\mathrm{nm}$. Uniform terminations, 
8. Surface analysis of the $\operatorname{PrB}_{6}$ (001) cleavage plane by scanning tunneling microscopy and spectroscopy

as shown in Fig. 8.2 (f) and Fig. 8.2 (j), are only observable on areas of a few $(\mathrm{nm})^{2}$ with a high density of defects. Scanning tunneling spectroscopy (STS) was utilized to resolve the LDOS for all ordered terminations. Local $I(V)$ curves were acquired and the differential conductance $d I / d V(V)$ is used as an approximation for the LDOS for comparison with PES and DFT results. Figure 8.2 (c) shows the differential conductance around the Fermi energy of the chainlike $(2 \times 1)$ reconstruction, which exhibits a finite conductance at the Fermi level and two distinct features at 0.2 and $-0.2 \mathrm{eV}$. In the energy range of $\pm 1.5 \mathrm{eV}$, the differential conductance is rather parabola shaped; see Fig. 8.6 in the Appendix. We expect that the $4 f$-related states are rather localized and therefore their wave function does not exceed far into the vacuum. To potentially address these states, we moved the tip closer to the surface by reducing the bias voltage set point. Figure 8.2 (d) shows a spectrum performed with a smaller bias voltage set point of $0.6 \mathrm{~V}$. Here, an additional peak in the electronic structure appears at $-1.1 \mathrm{eV}$.

Termination 2 is shown in Fig. 8.2 (e) and a CCT image is presented in Fig. 8.2 (f). The tunneling spectrum in Fig. 8.2 (g) in the range of $\pm 0.4 \mathrm{~V}$ shows, apart from surface metallicity, two features at about $\pm 0.2 \mathrm{eV}$. Additionally, a further spectral feature occurs at $-0.7 \mathrm{eV}$; see Fig. 8.2 (h).

The third termination, as seen in Fig. 8.2 (i) and Fig. 8.2 (j), shows only two spectral features at $\pm 0.2 \mathrm{~V}$; see Fig. 8.2 (k). Opposite to the previous data set of termination 2, no peak at $-0.7 \mathrm{eV}$ is observed, which is shown in Fig. 8.2 (1).

The atomic corrugation of the chainlike $(2 \times 1)$ reconstruction does not noticeably depend on the applied bias voltage; see Fig. 8.7 in the Appendix. Similarly, for termination 3 , the symmetry remains constant upon changing the bias voltage, which is shown in the Appendix as well; see Fig. 8.9.

In contrast, termination 2 shows a more complex corrugation dependency on the applied bias voltage. Figure 8.3 displays a multibias data set obtained at $0.1 \mathrm{nA}$ and $\pm 1, \pm 0.4$, and $\pm 0.1 \mathrm{~V}$. The atomic corrugation exhibits a $(1 \times 1)$ structure at a set point of $1 \mathrm{~V}$ and $0.1 \mathrm{nA}$. Reducing the bias voltage to $0.4 \mathrm{~V}$ leads to a rather ellipselike atomic corrugation, which is further elongated at $0.1 \mathrm{~V}$. This effect is even more pronounced for negative bias voltages. At -0.1 and $-0.4 \mathrm{~V}$, the atomic corrugation appears as stripes and with barely any modulation in the fast-scan axis. At $-1 \mathrm{~V}$, the high noise level dominates the CCT image. In conclusion, a 
stripelike corrugation is observed closer to the Fermi level and for the occupied states. A template average of this multibias data set can be seen in the Appendix; see Fig. 8.11.

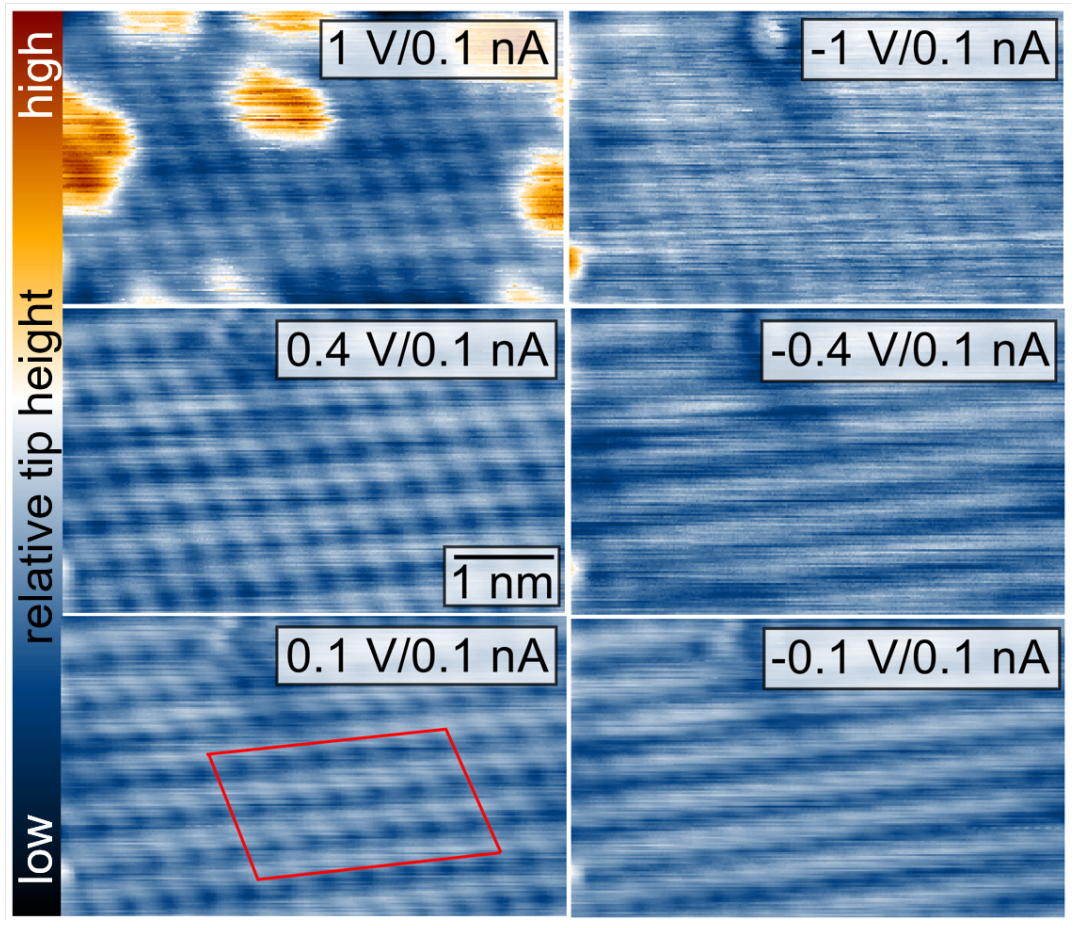

Figure 8.3: Multibias image of the stripelike reconstructed Pr termination taken at $0.1 \mathrm{nA}$ and $\pm 1, \pm 0.4$, and $\pm 0.1 \mathrm{~V}$. A template topography was made for each CCT image; see Fig. 8.11 and the respective discussion.

Please note that for the $1 \mathrm{~V} \mathrm{CCT}$, the atomic corrugation is partly covered with clusterlike protrusions. These defects become seemingly transparent at lower bias voltages, which is discussed in the Appendix in Fig. 8.5.

By reducing the bias voltage, the tip is brought closer to the surface. Another control of the tip-surface distance can be done by varying the tunneling current. By increasing the tunneling current by $50 \%$ to $0.15 \mathrm{nA}$, a stripy appearance can already be observed at $1 \mathrm{~V}$; see Fig. 8.4 and Fig. 8.10 in the Appendix. At $0.4 \mathrm{~V} / 0.15 \mathrm{nA}$, the atomic corrugation is even more stripelike shaped. At $-0.4 \mathrm{~V} / 0.15 \mathrm{nA}$, neither the $(1 \times 1)$ nor the stripes, but a rather complex corrugation, is observed. The corrugation becomes stripelike again at the set point of $-1 \mathrm{~V} / 0.15 \mathrm{nA}$. Figure 8.11 in the Appendix shows a template average of this 
8. Surface analysis of the $\operatorname{PrB}_{6}$ (001) cleavage plane by scanning tunneling microscopy and spectroscopy

multibias data set as well. Please note additionally that the multibias images in Fig. 8.3 and Fig. 8.4 were taken on two different samples with two different tunneling tips.

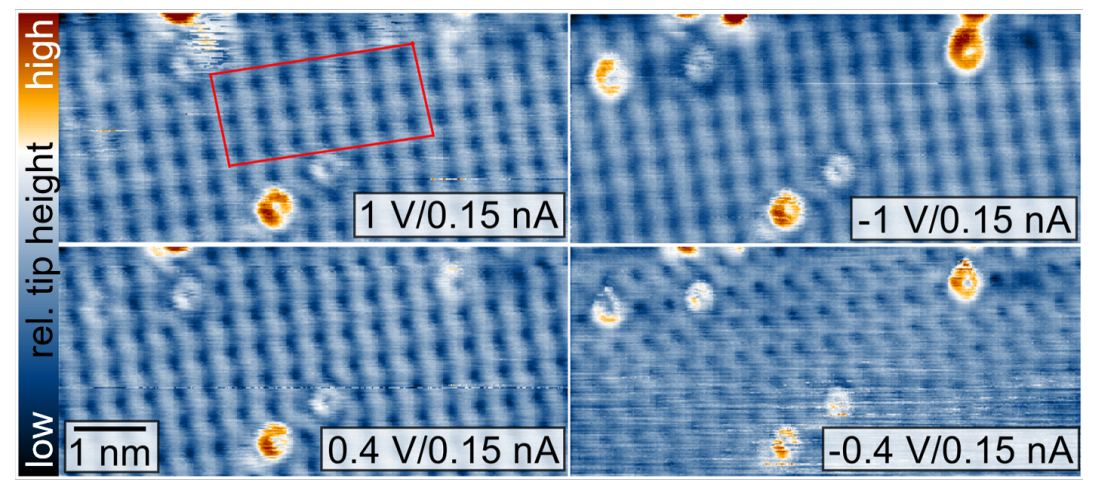

Figure 8.4: Multibias image of the stripelike reconstructed Pr termination taken at $0.15 \mathrm{nA}$ and $\pm 1, \pm 0.4 \mathrm{~V}$. A template topography was made for each CCT image; see Fig. 8.11 and the respective discussion.

\section{Discussion}

In summary, the STM experiments revealed the presence of three different ordered surface terminations. A chainlike $(2 \times 1)$ reconstruction has been observed for $\mathrm{LaB}_{6}$ [12] and $\mathrm{SmB}_{6}[45,67,103,46,64]$ as well, and has been assigned as parallel rows of rare-earth ions on top of $\mathrm{a}_{6}$ lattice. The long-range stability of this reconstruction is explained by avoiding the buildup of an electrical potential since it is made from alternating positively charged $\mathrm{Pr}$ and negatively charged $\mathrm{B}_{6}$ units. Consequently, we assign the observed chainlike $(2 \times 1)$ reconstruction as half-terminated Pr rows.

The tunneling spectra of this surface show two feature near $E_{\mathrm{F}}$, namely, at 0.2 and $-0.2 \mathrm{eV}$. Our previous study on the $\mathrm{LaB}_{6}(001)$ cleavage plane revealed a surface resonance of the chainlike $(2 \times 1)$ reconstruction below $E_{\mathrm{F}}$ stemming from the boron dangling bonds [12]. Here, we propose that the observed feature at $-0.2 \mathrm{eV}$ has the same origin. This idea needs to be confirmed by, e.g., DFT calculations. By moving the tip closer to the surface, an additional feature at 
$-1.1 \mathrm{eV}$ arises, which we tentatively describe as a $4 f$-related feature. This finding is consistent with photoemission experiments [61, 60] and bulk calculations [78] proposing the presence of $4 f$ states in this energy range. But by comparing photoemission results with the STS presented here, one has to take into account that PES typically averages over several $(\mu \mathrm{m})^{2}$. On the other hand, ordered surface terminations have been found only on a spatial scale of a few $(\mathrm{nm})^{2}$ and the majority of the encountered surface areas appear rather disordered. Furthermore, bulk calculations are not sufficient to describe the surface electronic structure due to the presence of boron dangling bonds, as seen, for instance, for $\mathrm{LaB}_{6}$ [12] and $\mathrm{EuB}_{6}$ [68]. Therefore, we cannot unambiguously exclude that the peak at $-1.1 \mathrm{eV}$ has a different orbital origin, e.g., it is a boron dangling bond state.

For termination 2, its most prominent spectral feature is the $d I / d V(V)$ peak at $-0.7 \mathrm{eV}$, which is again energetically close to the PES and DFT results for $4 f$ related states. Additionally, two spectral features $\pm 0.2 \mathrm{eV}$ around $E_{\mathrm{F}}$ are present.

Interestingly, the atomic corrugation deviates from a $(1 \times 1)$ structure. This has become apparent when moving the tip closer to the surface, either by bias voltage reduction or an increase of the tunneling current. The atomic corrugation develops to the rather stripelike appearance in one crystallographic direction and stays well modulated in the perpendicular crystallographic direction. As this stripelike reconstruction emerges for close tip-sample distances, we propose that it also originates from the $4 f$ electrons. Combining these findings, it is likely that this surface termination is Pr terminated since the $4 f$ electrons are hosted by the $\mathrm{Pr}$ ions. As reported, short-range magnetic ordering emerges below $\approx 20 \mathrm{~K}$ [76] close to boron defects [3]. Strictly speaking, boron defects are created by the cleavage itself at the surface. Therefore, the occurrence of the stripelike reconstruction at the surface might have a magnetic origin. Interestingly, the formation of local moments at a hexaboride surface has been observed on the $\mathrm{EuB}_{6}(001)$ cleavage plane [65]. But to this point, the underlying mechanism of the stripelike appearance needs further investigation to clarify whether it is an orbital rearrangement or whether it is induced by magnetic order.

The third termination shows a simple $(1 \times 1)$ symmetry and has two peaks in the differential conductance at $\pm 0.2 \mathrm{eV}$. Following the arguments above, this surface has to be $\mathrm{B}_{6}$ terminated. This is supported by the absence of any peaked 
8. Surface analysis of the $\operatorname{PrB}_{6}$ (001) cleavage plane by scanning tunneling microscopy and spectroscopy

spectral weight below $E_{\mathrm{F}}$ at $-0.7 \mathrm{eV}$. Furthermore, if the stripelike appearance would originate from an ordering of the magnetic moments, it is reasonable that it is not seen on a $B_{6}$ terminated surface.

Nevertheless, complementary techniques such as angle-resolved photoemission experiments or electronic structure calculations, which include different surface terminations, are required to unambiguously clarify the chemical nature of these observed terminations and their spectral properties.

\section{Conclusion}

$R \mathrm{~B}_{6}$ is a fascinating class of materials exhibiting diverse physical properties and understanding the surface physics is key for their technological utilization. In this paper, we have shown an atomic-scale study of the antiferromagnetic $4 f$ electron system $\operatorname{PrB}_{6}$. By investigating the (001) cleavage plane using STM and STS at low temperature under UHV conditions, we found, in addition to a widely rough and defect-rich surface, three ordered surface morphologies hosting distinctive structural and spectral features.

We thank F. Sohn and P. E. Blöchl for enlightening discussions. The orientation of the single crystals via gamma ray diffraction was carried out in the group of G. Eckold by F. Ziegler and P. Kirscht at the Institut für Physikalische Chemie, University of Göttingen. We gratefully acknowledge financial support by the DFG Grant No. WE1889/10-1. The work at the University of Warwick was supported by the EPSRC, UK, through Grant No. EP/T005963/1.

\section{Appendix}

\section{Disordered morphology}

On the $\operatorname{PrB}_{6}$ (001) cleavage plane, atomically ordered surface areas have to be searched for and occur rather infrequently. In most cases, the surface appears to be rough and rather disordered. In the following, some of these disordered morphologies are examined. 

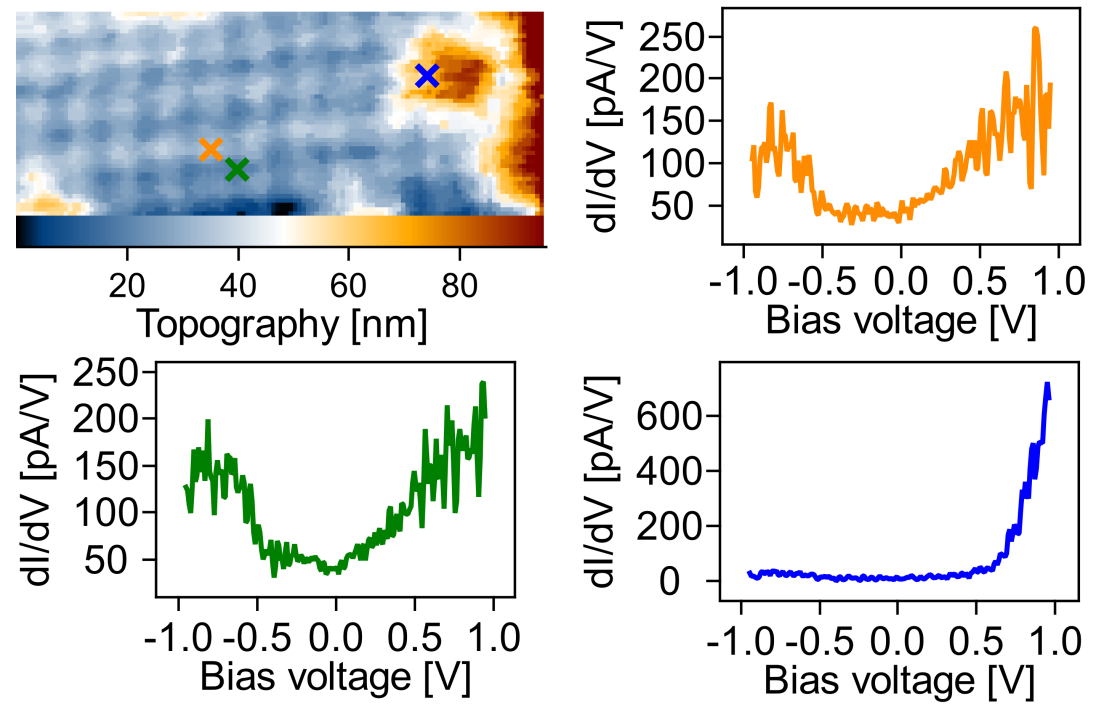

Figure 8.5: On the CCT image taken at $1 \mathrm{~V} / 0.1 \mathrm{nA}$, spatially resolved spectroscopy measurements were performed. The orange spectrum was taken on top of an atomic protrusion, the green spectrum on a hollow site, and the blue spectrum on a disordered protrusion. The spectra shown were obtained without lock-in amplification and are averaged over $3 \times 3$ individual $d I / d V$ curves.

Locally resolved tunneling spectroscopy $d I / d V(V, x, y)$ was made on the same Pr terminated area of Fig. 8.3 and three exemplary spectra are shown in Fig. 8.5. The orange spectrum was taken on an atomic protrusion and the spectral features at -0.2 and $-0.7 \mathrm{eV}$ coincide with the previously shown lock-in spectra in Fig. 8.2 (g) and Fig. 8.2 (h). The spectrum at the hollow site, i.e., the green $d I / d V$ curve, is similar to the $d I / d V$ curve taken on top of the atomic corrugation. The spectrum taken at the disordered protrusion, i.e., the blue $d I / d V$ curve, shows a different behavior, namely, high $d I / d V$ values above $0.6 \mathrm{~V}$ and almost vanishing $d I / d V$ values below. Therefore, we hypothesize that these protrusions are addressed at bias voltages above $0.6 \mathrm{~V}$, whereas at smaller bias voltages, the atomic lattice is addressed. This is in agreement with the apparent disappearance of the cluster in the multibias data set; see Fig. 8.3.

The high number of defects and the mostly disordered occurring surface are in agreement with the observations on cleaved $\mathrm{LaB}_{6}$ [12] and $\mathrm{SmB}_{6}$ [45]. The hexaborides are made of a fully three-dimensional covalent binding network and a natural cleavage plane is missing. Therefore, the cleavage can happen in between 
8. Surface analysis of the $\operatorname{PrB}_{6}$ (001) cleavage plane by scanning tunneling microscopy and spectroscopy

or within the octahedra and the surface tends to appear rough on the atomic scale. The ordered surface terminations, on the other hand, are often polar. Even for the nonpolar chainlike $(2 \times 1)$ reconstruction, the atomic chains are only of a few $\mathrm{nm}$ length and frequently interrupted by defects. Consequently, we find the surface to be rather vulnerable. Furthermore, noise has been a significant problem in our data acquisition on hexaborides. Already in our previous study on $\mathrm{LaB}_{6}$ [12], we stated in the discussion that "we find rather unstable tunneling conditions for negative bias voltages." Additionally, we had difficulties obtaining tunneling spectra for a larger range than $\pm 1 \mathrm{~V}$. Similar behavior is found for $\operatorname{PrB}_{6}$.

On both uniform terminations, ringlike defects are present (see Fig. 8.4 and Fig. 8.9). Such defects have been observed on cleaved $\mathrm{SmB}_{6}$ as well and were attributed to disrupted boron structures $[86,71]$. Here, on cleaved $\operatorname{PrB}_{6}$, we believe that the same origin seems likely.

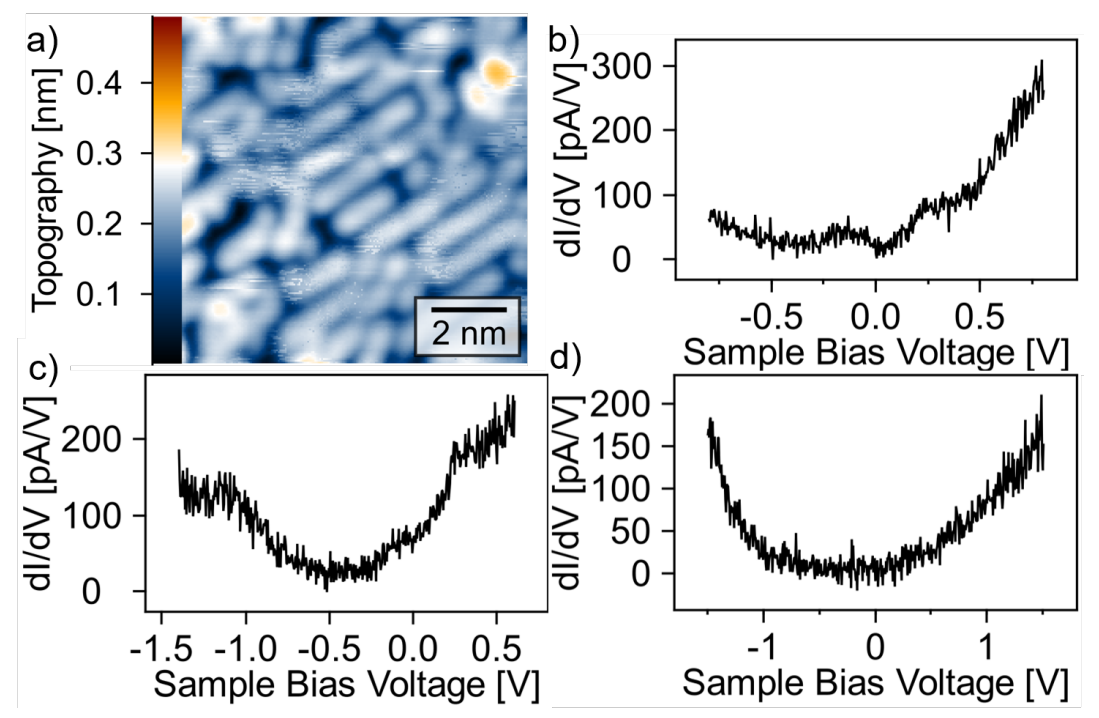

Figure 8.6: (a) CCT of the chainlike $(2 \times 1)$ reconstruction, taken at $0.4 \mathrm{~V}$ and $0.1 \mathrm{nA}$. (b) Tunneling spectrum taken at $0.8 \mathrm{~V}, 0.1 \mathrm{nA}$, and $V_{\text {mod }}=5 \mathrm{mV}$. (c) Tunneling spectrum taken at $0.6 \mathrm{~V}, 0.1 \mathrm{nA}$, and $V_{\mathrm{mod}}=5 \mathrm{mV}$. (d) Tunneling spectrum taken at $1.5 \mathrm{~V}, 0.1 \mathrm{nA}$, and $V_{\text {mod }}=5 \mathrm{mV}$. All spectra were taken on top of an atomic chain. 


\section{Supporting data of the ordered surface terminations}

Figure 8.6 shows a spectroscopy data set of the chainlike $(2 \times 1)$ reconstruction, and in Fig. 8.7 a multibias data set of the chainlike $(2 \times 1)$ reconstruction is shown. As stated above, no corrugation change can be observed for the applied bias voltages.

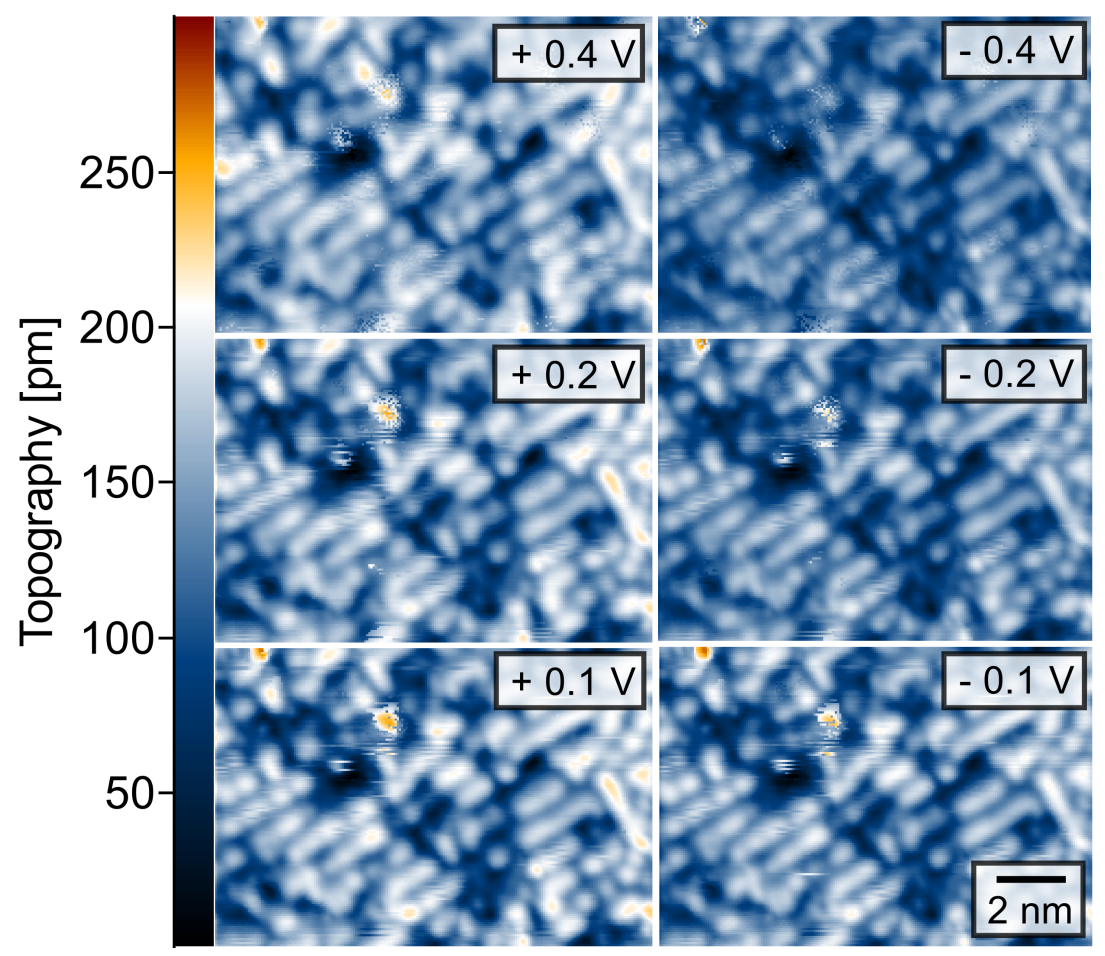

Figure 8.7: Multibias data set of the chainlike $(2 \times 1)$ reconstruction. The images were obtained at $\pm 0.4, \pm 0.2$, and $\pm 0.1 \mathrm{~V}$, and at a tunneling current of $0.1 \mathrm{nA}$. 
8. Surface analysis of the $\operatorname{PrB}_{6}$ (001) cleavage plane by scanning tunneling microscopy and spectroscopy

On a cubic surface, no preference between a $(2 \times 1)$ or $(1 \times 2)$ orientation should be present. To investigate the influence of surface defects on the major alignment, Fig. 8.8 shows two steps between chainlike $(2 \times 1)$ reconstructed areas. In both cases, the step height is one bulk lattice constant [see Fig. 8.8 (c) and Fig. 8.8 (f)] and the step edge proceeds vertically [see Fig. 8.8 (a) and Fig. 8.8 (d)]. Although the surrounding conditions for the reconstruction seem to be identical, the atomic chains align either $(2 \times 1)$ or $(1 \times 2)$ throughout the step edge. Consequently, we find that the formation of the predominant alignment is independent from the orientation of step edges.
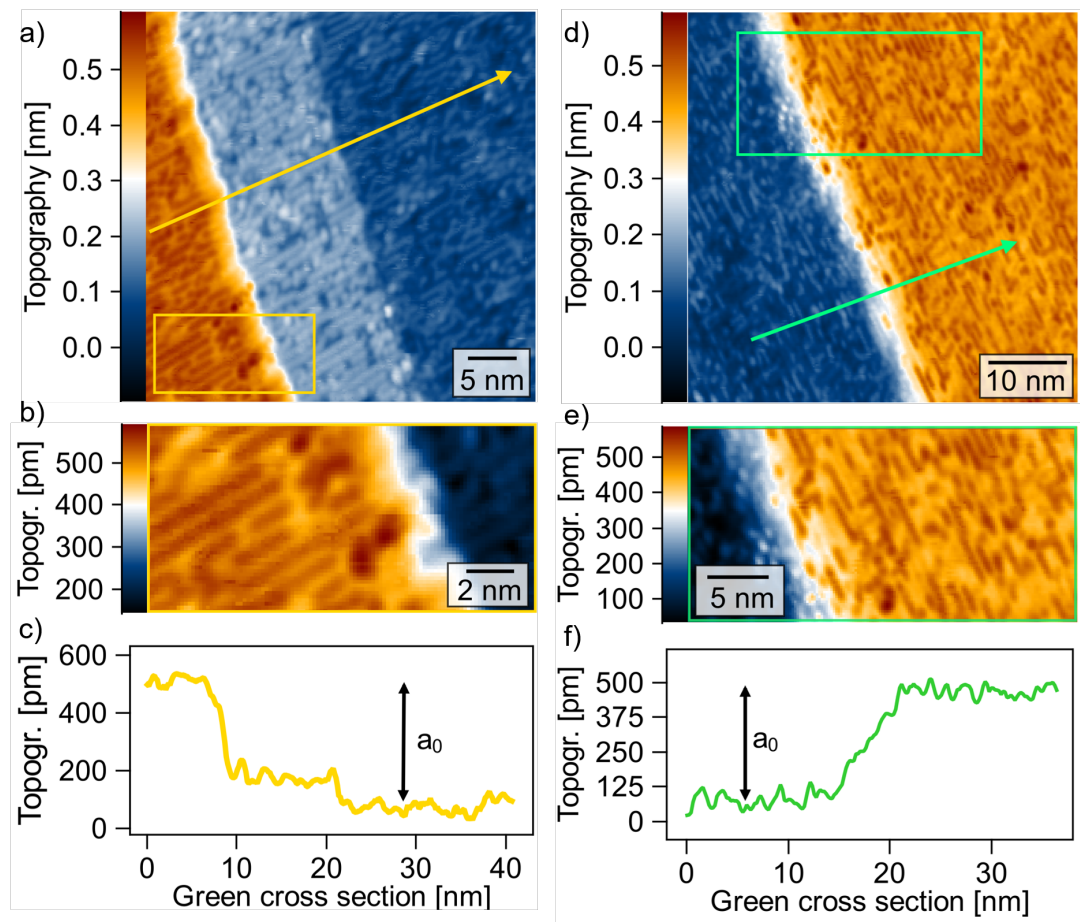

Figure 8.8: (a) CCT taken at $0.4 \mathrm{~V} / 0.1 \mathrm{nA}$. The predominant alignment of the rows is perpendicular to the step edge. (b) Magnification of the golden-colored area in (a). (c) Cross section as indicated by the arrow in (a). (d) The predominant alignment can be arranged parallel to the step edges too, as evident in the highresolution image seen in (e). CCT in (d) taken at $0.4 \mathrm{~V} / 0.1 \mathrm{nA}$. (f) Cross section as indicated by the arrow in (d). Please note that in (a), a double-tip artifact is present. 
We do not observe a corrugation change of the uniform $\mathrm{B}_{6}$ termination upon varying the bias voltage. Figure 8.9 shows a multibias data set of this surface morphology taken at $0.1 \mathrm{nA}$ and $\pm 0.8, \pm 0.4$, and $\pm 0.1 \mathrm{~V}$.

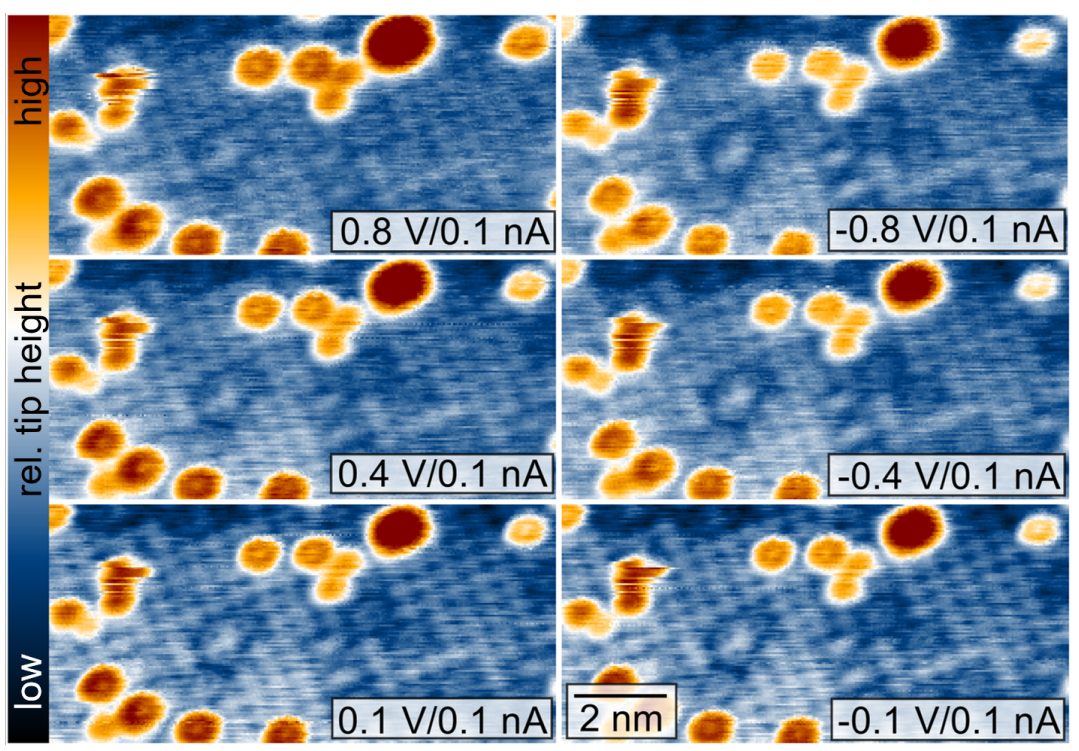

Figure 8.9: Multibias CCTs taken at $0.1 \mathrm{nA}$ and $\pm 0.8, \pm 0.4$, and $\pm 0.1 \mathrm{~V}$ on the $(1 \times 1)$ reconstructed $B_{6}$ surface. 
8. Surface analysis of the $\operatorname{PrB}_{6}$ (001) cleavage plane by scanning tunneling microscopy and spectroscopy

In Fig. 8.10, two CCTs of the uniform Pr termination are shown, which were taken on the same area as Fig. 8.4. For both images, the bias voltage was set to $1 \mathrm{~V}$, but the tunneling current for the left image is $0.1 \mathrm{nA}$ and for the right image is $0.15 \mathrm{nA}$. For both CCT images, a template average was made as well. The apparent corrugation becomes more stripelike by increasing the tunneling current, as evident from the template topographies shown.

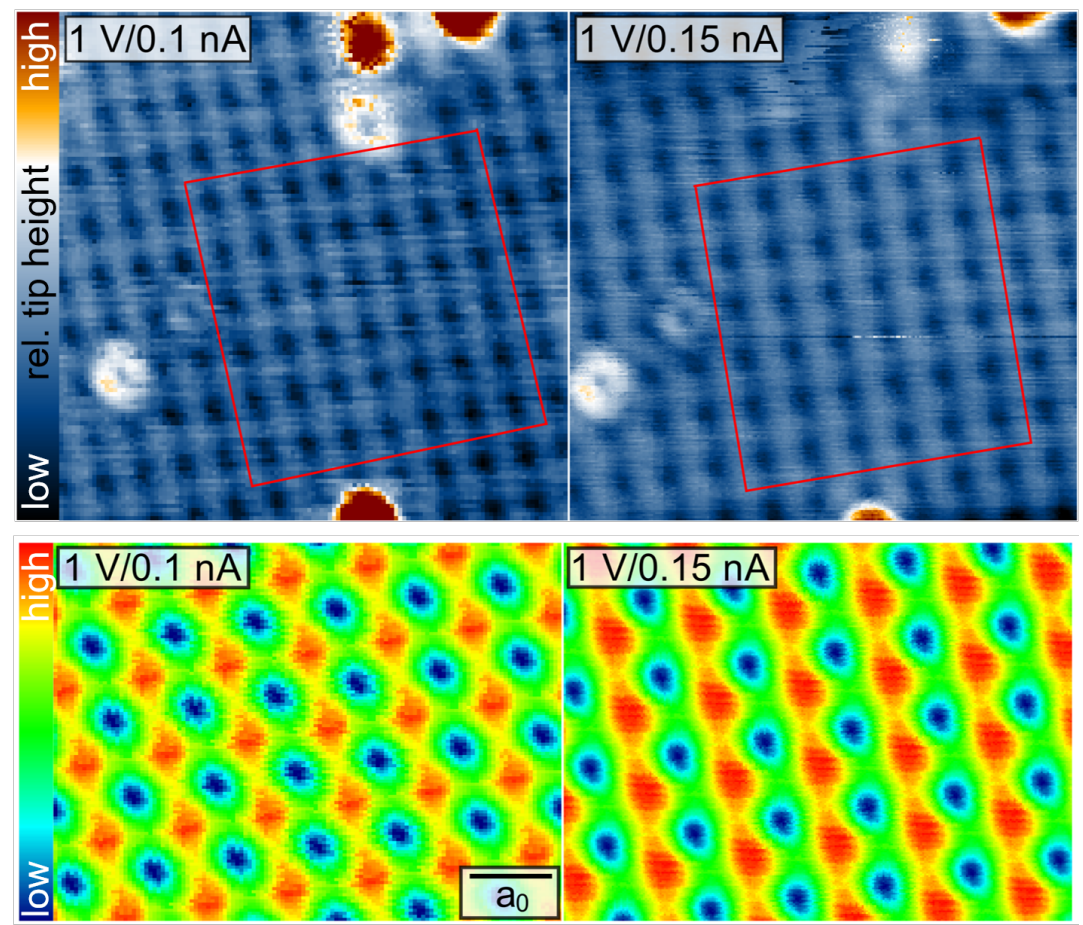

Figure 8.10: Top left: CCT image of the Pr terminated surface taken at $1 \mathrm{~V} / 0.1 \mathrm{nA}$. Top right: CCT image of the Pr terminated surface taken at $1 \mathrm{~V} / 0.15 \mathrm{nA}$. The reddish enclosed area marks the area where the template average was performed. Bottom left: Template topography made from the CCT on the top left. Bottom right: Template topography made from the CCT on the top right. 
Figure 8.11 shows the template-averaged topographies of Fig. 8.3 at the top and of Fig. 8.4 at the bottom, respectively. Here, an averaged unit cell is calculated for each individual CCT from an area, which is enclosed in red in one exemplary CCT image in both Fig. 8.3 and Fig. 8.4.

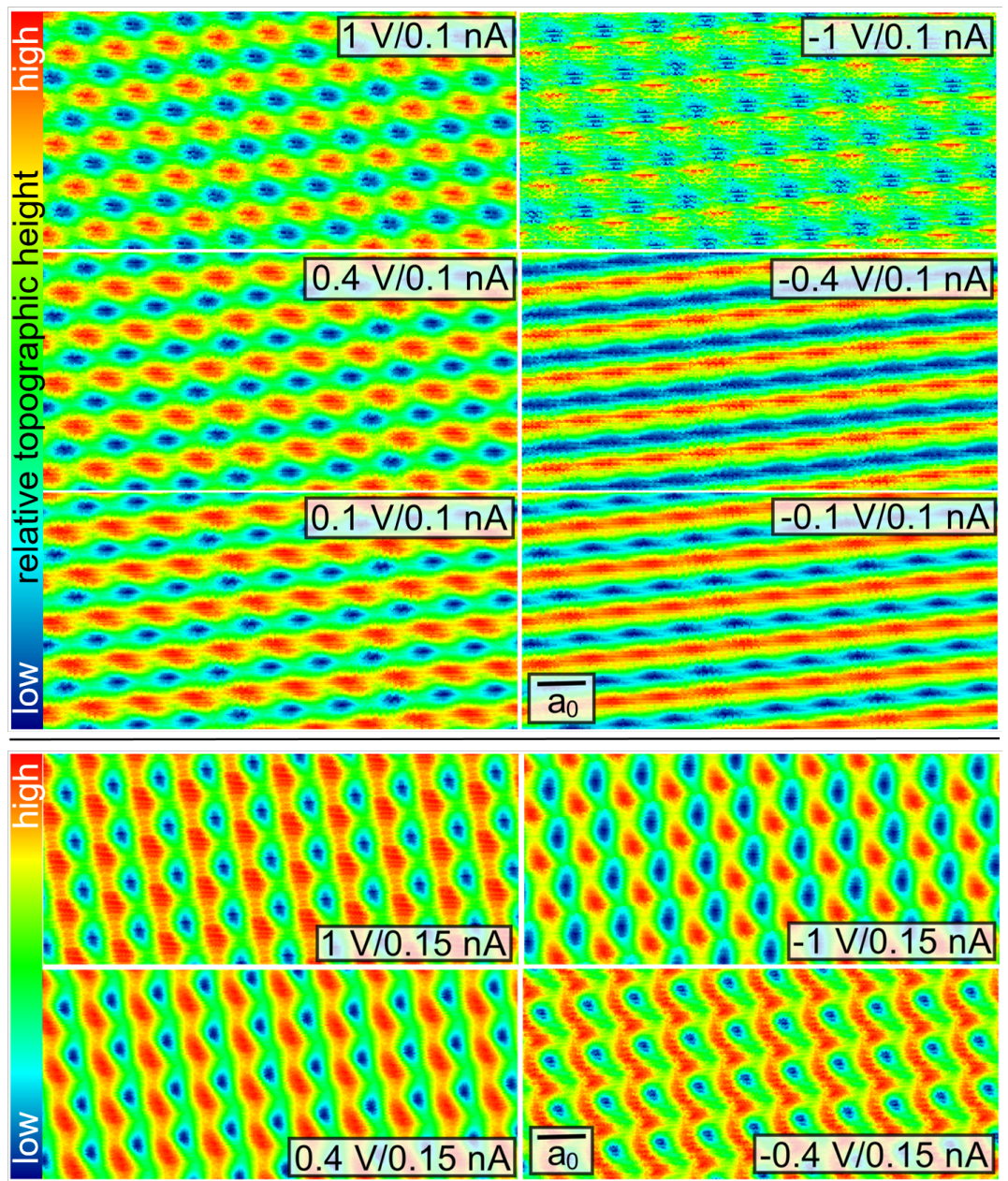

Figure 8.11: Template topographies of Fig. 8.3 at the top and of Fig. 8.4 at the bottom. The set points are indicated in the respective images. 
8. Surface analysis of the $\operatorname{PrB}_{6}$ (001) cleavage plane by scanning tunneling microscopy and spectroscopy 


\section{Chapter 9}

\section{Comparison of the $\operatorname{LaB}_{6}$ and $\operatorname{PrB}_{6}$ results}

In the following chapter our results on the surface structure and electronic properties of $\mathrm{LaB}_{6}$ and $\mathrm{PrB}_{6}$ are summarized and compared to each other. As a general remark, the $\mathrm{LaB}_{6}$ and $\mathrm{PrB}_{6}$ (001)-cleavage planes appear both very rough on an atomic scale and by far most of the encountered surface areas are disordered. Finding atomically ordered surfaces is a severe challenge. The main reason for this observation is surely the crystal structure itself. The boron bonds construct a fully three-dimensional binding network and a natural cleavage plane is missing in hexaborides. Hence, disordered surfaces have been reported for $\mathrm{SmB}_{6}$ [45] as well. Research on hexaboride surfaces is not only a challenge for material science and correlation physics, but also requires a tremendous amount of patience.

\subsection{Chainlike $(2 \times 1)$ reconstruction}

Starting with $\mathrm{LaB}_{6}$, we found in a joint STM, LEED and DFT study, that a chainlike $(2 \times 1)$ reconstruction is present on a local scale. The atomic rows of the reconstruction are only arranged parallel to each other in the vicinity of step edges. At the step edge, the chains tend to order perpendicular to the step edge, whereas farther away from the step the chains develope a rather labyrinthlike pattern. Our DFT calculations showed that this reconstruction can be understood as La ions on 
top of a complete $\mathrm{B}_{6}$ layer.

On $\operatorname{PrB}_{6}$, we found a chainlike $(2 \times 1)$ reconstruction as well. The rows are only of few nm length, which is comparable to the rows on $\mathrm{LaB}_{6}$. The atomic rows on $\mathrm{PrB}_{6}$ on the other hand can have a predominant alignment over hundreds of $\mathrm{nm}$ independent from the presence of step edges. As shown in Fig. 8.8, the chains can be arranged parallel or perpendicular to the step edge.

\subsection{Uniform terminations}

Furthermore, we observed two uniform terminations on the $\operatorname{PrB}_{6}$ (001)-cleavage plane. These terminations are assigned as complete rare earth and $\mathrm{B}_{6}$ layers in accordance to the observations on cleaved $\mathrm{SmB}_{6}$ and $\mathrm{EuB}_{6}$ [103]. However, these uniform terminations can only be found rarely and on a small spatial scale of few $(\mathrm{nm})^{2}$ accompanied with a high defect density. This is consistent with the polar nature of the uniform terminations and the missing of a natural cleavage plane, as seen for $\mathrm{SmB}_{6}$ as well [46].

$\mathrm{On} \mathrm{LaB}_{6}$ a uniform La termination has been reported for heated samples [58, 59]. Within the scope of this thesis, cleaved $\mathrm{LaB}_{6}$ was investigated and no uniform La or $\mathrm{B}_{6}$ terminations could be found. However, since STM is an highly local probe method, it cannot be excluded that small patches of uniform La or $\mathrm{B}_{6}$ may exist in particular surface areas. This might be anticipated from the surface step we observed on $\mathrm{LaB}_{6}$, see Fig. 7.11. Here, an apparent step height of $1.3 \AA$ marked the transition from a chainlike $(2 \times 1)$ reconstruction to a supposedly rather boron rich termination. Nevertheless, as already theoretically predicted [74], the uniform La or $\mathrm{B}_{6}$ terminations are less stable than a chainlike $(2 \times 1)$ reconstruction on $\mathrm{LaB}_{6}$. 


\subsection{Bulk and surface defects}

We found more atomically ordered areas on $\operatorname{PrB}_{6}$ than on $\mathrm{LaB}_{6}$. To this point, we are not sure whether this is stemming from an intrinsic material property or if it is just a coincidence. However, Otani et al. [57] noted that the crystal growth of $\mathrm{LaB}_{6}$ led to more imperfections than for $\mathrm{PrB}_{6}$. And indeed, in our present crystals, we found that the quality of the $\operatorname{PrB}_{6}$ single crystal was remarkably higher, as seen in Chapter 4. The mosaicity, a control parameter for the lattice mismatch, was notably increased for the $\mathrm{LaB}_{6}$ crystal. The higher number of crystal defects might have influenced the cleavage process leading to fewer atomically ordered surface areas.

Nevertheless, as stated above, both $\mathrm{LaB}_{6}$ and $\operatorname{PrB}_{6}$ surfaces appear rather rough on the atomic scale due to the lack of a natural cleavage plane. As argued for $\mathrm{SmB}_{6}$, the cleavage through a boron octahedron could lead to ring-like surface defects $[71,86]$. In the present thesis, comparable ring-like defects were observed on $\mathrm{PrB}_{6}$ as well, see Fig. 8.4 and Fig. 8.9.

The ordered surface areas are often polar and consequently they extend only over few $(\mathrm{nm})^{2}$. Even for the non-polar chainlike $(2 \times 1)$ reconstruction on both $\mathrm{LaB}_{6}$ and $\operatorname{PrB}_{6}$, the chains are only of few atoms length until they get interrupted by surface defects. This apparent vulnerability of the surfaces surely resulted in the elevated noise level in the data and occasionally led to rather unstable tunneling conditions. Therefore, multi-bias imaging and tunneling spectroscopy, both measurement modes with a fast change of bias voltage magnitude and polarity, oftentimes accidentally caused surface or tip modifications. 


\subsection{Spectral properties}

Spatially resolved STS on the chainlike $(2 \times 1)$ reconstruction of $\mathrm{LaB}_{6}$ reveal a surface resonance at $-0.6 \mathrm{eV}$, see Fig. 7.5. Our DFT calculations interpreted this spectral feature as B2 $p$ dangling bond state with La5d contribution, see Fig. 7.6. The calculated peak position however was predicted at $-0.2 \mathrm{eV}$. This apparent discrepancy of $0.4 \mathrm{eV}$ is quite satisfactory, especially since the goal of our calculation was a qualitative description of the experimentally observed spectral feature. However, a small shift of the observed spectral feature might be traced back to both, surface and bulk imperfections. Firstly, the atomic rows on $\mathrm{LaB}_{6}$ were only short-ranged and rather labyrinth-like arranged. Therefore, a deviation from the peak position of pristine La rows, as anticipated in the calculations, are understandable. Secondly, we attribute a small shift to a boron deficiency of the bulk material. Preferrential evaporation of boron leads to rather boron deficient crystals after the growth [56]. Consequently, the aspect ratio of $\mathrm{La} / \mathrm{B}$ is slightly higher, which leads to a surplus of charge carriers. Hence, the Fermi-level may be shifted towards higher energies, so that our observed B2 $p$ peak appears at smaller energies.

On $\operatorname{PrB}_{6}$, we observed a peak at $-0.2 \mathrm{eV}$ on the chainlike $(2 \times 1)$ reconstructed surface, too. Since the surface resonance on $\mathrm{LaB}_{6}$ is mainly a boron danglingbond feature, we believe that the aforementioned spectral feature on $\operatorname{PrB}_{6}$ has the same origin. Nevertheless, DFT calculations for the $\operatorname{PrB}_{6}$ surface are obligatory to reliably clarify the orbital origin.

Furthermore, we observed a peak at $-1.1 \mathrm{eV}$ on the $\operatorname{PrB}_{6}$ chainlike $(2 \times 1)$ reconstruction when moving the tip closer to the surface. PES suggested the presence of the $4 f$ electrons in this energy region [61,60]. We believe, that the $4 f$ electrons are rather localized and their wave function does not exceed far into the vacuum. Hence, these states would be only addressable by a tip height reduction.

On $\mathrm{LaB}_{6}$, we have seen an interesting distance dependance as well, namely for the different La5 $d$ states. Our calculations showed, that the La5 $d_{z}$ states on the unoccupied side are reaching far into the vacuum and hence they are addressable at positive bias voltages. For negative bias voltages, the La5 $d_{x y}$ states are present, which are pointing in-plane towards the dangling bonds. Hence, their wave func- 
tion is not exceeding far into the vacuum and are not conveniently addressable with STM. DFT calculations of the $\operatorname{PrB}_{6}$ surface could clarify the orbital character of the $-1.1 \mathrm{eV}$ feature and subsequently resolve the decay behavior into the vacuum.

The electronic structure of the $\mathrm{B}_{6}$ termination on $\mathrm{PrB}_{6}$ is rather simple, as it showed only two spectral peaks at $\pm 0.2 \mathrm{eV}$ around $E_{\mathrm{F}}$.

Electronically more complex than the uniform $\mathrm{B}_{6}$ termination is the uniform Pr termination on $\operatorname{PrB}_{6}$. Apart from the two peaks at $\pm 0.2 \mathrm{eV}$ around $E_{\mathrm{F}}$ another spectral feature at $-0.7 \mathrm{eV}$ occurs, which we preliminarily interpret as $4 f$ resonance in agreement with the PES data [61,60].

However, the observed spectral features at $-0.7 \mathrm{eV}$ for the Pr termination and the spectral feature at $-1.1 \mathrm{eV}$ for the chainlike $(2 \times 1)$ reconstruction on $\mathrm{PrB}_{6}$ could both result from a boron dangling bond states as well. Resolving the electronic surface structure of $\operatorname{PrB}_{6}$ clearly requires complementary investigations such as angle-resolved photoemission and DFT slab calculations.

\subsection{Corrugation dependance on the bias voltage}

The overall chainlike appearance of the $\operatorname{PrB}_{6}(2 \times 1)$ reconstruction does not noticably change by varying the applied bias voltage. For $\mathrm{LaB}_{6}$, our calculations suggested as well, that regardless of positive or negative bias voltages, the chainlike appearance remains in CCT images.

For the $\mathrm{B}_{6}$ terminated surface of $\operatorname{PrB}_{6}$, the simple $(1 \times 1)$ structure remains upon changing the bias voltage.

A fascinating feature of the unifrom Pr termination is the complex bias voltage dependence of the atomic corrugation as observed in multi-bias imaging. Moving the tip closer to the surface by either a bias voltage reduction or an increase of the tunneling current leads to a change from a simple $(1 \times 1)$ symmetry to a rather stripelike reconstruction. Since the $4 f$ electrons are hosted by the Pr atoms, we hypothesize that the stripelike appearance originates from the $4 f$ electrons. Please note, that at our experiment temperature of $8 \mathrm{~K}$ the sample is within the spin-glass regime and short-range ordering is present. It seems likely, that the stripelike appearance is connected to the above mentioned short-range ordering. 


\subsection{Spatially resolved LEED}

We performed LEED experiments in an conventional setup on the $\mathrm{LaB}_{6}$ (001)cleavage plane and observed only $(1 \times 1)$ diffraction spots. By using the ULEED setup, which allows spatial resolution, we obseverd mostly $(1 \times 1)$ spots as well. Additionally, on some surface areas $(2 \times 1)$ or the by $90^{\circ}$ rotated $(1 \times 2)$ spots appeared. The reconstruction spots can be traced back to the chainlike $(2 \times 1)$ reconstruction found on $\mathrm{LaB}_{6}$. Since the majority of the $\mathrm{LaB}_{6}$ (001)-cleavage plane appears rather disordered, the frequently occuring $(1 \times 1)$ spots stem from the bulk periodicity due to the finite penetration depth of the electrons.

Similarly, we found mostly $(1 \times 1)$ spots on the $\operatorname{PrB}_{6}$ cleavage plane using the ULEED steup. Occasionally, $(2 \times 1)$ spots appeared, which we trace back to the chainlike $(2 \times 1)$ reconstruction.

To clarify if the stripelike reconstruction is a structural reconstruction formed below $20 \mathrm{~K}$, we performed temperature dependent ULEED at the $\operatorname{PrB}_{6}$ (001)cleavage plane as well. Again, at RT the surface showed mainly $(1 \times 1)$ spots and on one surface area $(2 \times 1)$ spots were found. Unfortunately, the lowest reachable temperature with the present setup was found to be $27 \mathrm{~K}$. At this temperature no notable change of the diffraction pattern could be observed and the origin of the stripelike reconstruction remains puzzeling. 


\section{Chapter 10}

\section{Outlook}

Hexaborides are a fascinating class of materials and understanding their surface properties is essential for present and future technological applications. In the present thesis, two atomic scale studies of the (001)-cleavage planes of $\mathrm{LaB}_{6}$ and $\mathrm{PrB}_{6}$ are displayed.

In the $\mathrm{LaB}_{6}$ surface study we have explicitly addressed the importance of the surface preparation for the resulting surface morphology. Furthermore, we have shown that the surface electronic structure largely differs from the bulk properties due to surface reconstruction and dangling bond states.

On the $\mathrm{PrB}_{6}$ (001)-cleavage plane we have found three ordered surface morphologies, which harbor unique structural and electronic characteristics. Unfortunately, no electronic structure calculations could have been made within our joint project to reliably describe the influence of the $4 f$ electrons on the $\operatorname{PrB}_{6}$ surface. However, we hope that the presented results will provide a valuable foundation to benchmark future calculations.

It is worth noting, that we have also conducted STM experiments on $\mathrm{CeB}_{6}$. However, the surface appeared very rough on the atomic scale and the results have not been displayed within this thesis. Therefore, we have to leave the STM investigation of $\mathrm{CeB}_{6}$ to other groups.

In summary it can be said, that each individual surface termination of a hexaboride can have altered physical properties compared to each other and compared to the respective bulk material. Another way to control the properties of hexa- 
borides is chemical doping. Substituting the rare earth elements within the $\mathrm{B}_{6}$ framework can be conveniently done, since all $R \mathrm{~B}_{6}$ share the same crystal structure [13]. For instance, magnetic and non-magnetic impurities have been used to investigate the suppression of surface states in $\mathrm{SmB}_{6}$ [36] and Fe and Gd doping in $\mathrm{SmB}_{6}$ has been utilized to enhance quasiparticle scattering [64]. The increasing interest in doped hexaborides also traces back to the goal of tailoring their thermal, electrical and optical properties [13]. Various ternary compounds have been synthesized, such as $(\mathrm{La}-\mathrm{Y}) \mathrm{B}_{6},(\mathrm{La}-\mathrm{Eu}) \mathrm{B}_{6},(\mathrm{La}-\mathrm{Ce}) \mathrm{B}_{6},(\mathrm{Eu}-\mathrm{Y}) \mathrm{B}_{6},(\mathrm{Ca}-\mathrm{Yb}) \mathrm{B}_{6},(\mathrm{Ca}-$ $\mathrm{Sm}) \mathrm{B}_{6}$ and many more [13].

Combining the versatile surfaces obtainable from cleaving and heating a single crystalline hexaboride with the seemingly endless possibilities of chemical substitution of the base material, the interest in hexaboride surface physics may never come to the end. 


\section{Bibliography}

[1] CP-PAW website. https://www2 .pt.tu-clausthal.de/paw/.

[2] K. Adachi, M. Miratsu, and T. Asahi. J. Mater. Res., 25(3):510-521, 2010.

[3] M. A. Anisimov, A. V. Bogach, V. V. Glushkov, S. V. Demishev, N. A. Samarin, N. Yu Shitsevalova, A. V. Levchenko, V. B. Filipov, A. V. Kuznetsov, and N. E. Sluchanko. Solid State Phenomena, 190:221-224, 2012.

[4] M. Aono, R. Nishitani, C. Oshima, T. Tanaka, E. Bannai, and S. Kawai. Surf. Sci., 86:631 $-637,1979$.

[5] M. Aono, C. Oshima, T. Tanaka, E. Bannai, and S. Kawai. J. Appl. Phys., 49(5):2761-2764, 1978.

[6] G. Balakrishnan, M. R. Lees, and D. McK. Paul. J. Cryst. Growth, 256(1):206 - 209, 2003.

[7] G. Balakrishnan, M. R. Lees, and D. McK. Paul. J. Magn. Magn. Mater, 272-276:601 602, 2004.

[8] J. Bardeen. Phys. Rev. Lett., 6(2):57-59, 1961.

[9] P. E. Blöchl. Phys. Rev. B, 50(24):17953-17979, 1994.

[10] C. H. Booth, J. L. Sarrao, M. F. Hundley, A. L. Cornelius, G. H. Kwei, A. Bianchi, Z. Fisk, and J. M. Lawrence. Phys. Rev. B, 63(22):224302, 2001.

[11] P. Buchsteiner. Scanning tunneling spectroscopy on the LaB6 and CeB6 (001)-surface. Master thesis, Georg-August-Universität Göttingen, 2016.

[12] P. Buchsteiner, F. Sohn, J. G. Horstmann, J. Voigt, M. Ciomaga Hatnean, G. Balakrishnan, C. Ropers, P. E. Blöchl, and M. Wenderoth. Phys. Rev. B, 100:205407, 2019.

[13] J. T. Cahill and O. A. Graeve. J. Mater. Res. Technol., 8(6):6321 - 6335, 2019.

[14] R. Car and M. Parrinello. Phys. Rev. Lett., 55(22):2471-2474, 1985.

[15] S. A. Chambers and L. W. Swanson. Surf. Sci., 131(2):385 - 402, 1983.

[16] L. Chao, L. Bao, W. Wei, and O. Tegus. Sol. Energy, 190:10 - 27, 2019. 
[17] C. J. Chen. Introduction to Scanning Tunneling Microscopy. Oxford University Press, New York, 2nd edition, 2008.

[18] J.-P. Colinge, C.-W. Lee, A. Afzalian, N. D. Akhavan, R. Yan, I. Ferain, P. Razavi, B. O'Neill, A. Blake, M. White, A.-M. Kelleher, B. McCarthy, and R. Murphy. Nat. Nanotechnol., 5(3):225-229, 2010.

[19] L. A. DuBridge. Phys. Rev., 31:236-243, 1928.

[20] M. Dzero, K. Sun, V. Galitski, and P. Coleman. Phys. Rev. Lett., 104:106408, 2010.

[21] M. Dzero, J. Xia, V. Galitski, and P. Coleman. Annu. Rev. Condens. Matter Phys., 7(1):249280, 2016.

[22] M. Enayat. Construction and Operation of a milli-Kelvin Spectroscopic Imaging STM for the study of Correlated electron Materials. PhD thesis, École Polytechnique Fédérale de Lausanne (EPFL-Switzerland), 2014.

[23] J. K. Garleff, M. Wenderoth, K. Sauthoff, R. G. Ulbrich, and M. Rohlfing. Phys. Rev. B, 70(24):245424, 2004.

[24] T. H. Geballe, B. T. Matthias, K. Andres, J. P. Maita, A. S. Cooper, and E. Corenzwit. Science, 160(3835):1443-1444, 1968.

[25] B. Goldstein and D. J. Szostak. Surf. Sci., 74(2):461 - 478, 1978.

[26] T. Gürel and R. Eryiğit. Phys. Rev. B, 82(10):104302, 2010.

[27] X. Guo-Liang, C. Jing-Dong, X. Yao-Zheng, L. Xue-Feng, L. Yu-Fang, and Z. Xian-Zhou. Chin. Phys. Lett., 26(5):056201, 2009.

[28] R. J. Hamers. Annu. Rev. Phys. Chem., 40(1):531-559, 1989.

[29] L. Harmsen. Charakterisierung der (100) Spaltflächen von LaB6 und CeB6. Bachelor thesis, Georg-August-Universität Göttingen, 2017.

[30] L. Harmsen. Scanning tunneling microscopy on the PrB6 (001)-surface. Master thesis, Georg-August-Universität Göttingen, 2019.

[31] M. Z. Hasan and C. L. Kane. Rev. Mod. Phys., 82:3045-3067, 2010.

[32] P. Hohenberg and W. Kohn. Phys. Rev., 136(3):B864-B871, 1964.

[33] F. M. Hossain, D. P. Riley, and G. E. Murch. Phys. Rev. B, 72:235101, 2005.

[34] V. I. Ivashchenko, P. E. A. Turchi, V. I. Shevchenko, N. R. Medukh, J. Leszczynski, and L. Gorb. Physica B, 531:216 - 222, 2018.

[35] K. Iwashita, T. Matsumura, K. Segawa, and S. Kunii. Physica B, 186-188:636 - 638, 1993.

[36] L. Jiao, S. Rößler, D. Kasinathan, P. F. S. Rosa, C. Guo, H. Yuan, C.-X. Liu, Z. Fisk, F. Steglich, and S. Wirth. Sci. Adv., 4(11):eaau4886, 2018. 
[37] T. Kasuya. Europhys. Lett., 26(4):277-281, 1994.

[38] W. Kohn and L. J. Sham. Phys. Rev., 140(4):A1133-A1138, 1965.

[39] S. Kunii, T. Kasuya, K. Kadowaki, M. Date, and S. B. Woods. Solid State Commun., 52(7):659-661, 1984.

[40] J. M. Lafferty. J. Appl. Phys., 22(3):299-309, 1951.

[41] M. G. Lagally. J. Vac. Sci. Technol., A, 21(5):S54-S63, 2003.

[42] M. N. Leuenberger and D. Loss. Nature, 410(6830):789-793, 2001.

[43] H. C. Longuet-Higgins and M. De V. Roberts. Proc. R. Soc. London, Ser. A, 224(1158):336$347,1954$.

[44] H. Lüth. Solid Surfaces, Interfaces and Thin Films. Springer, Berlin, Heidelberg, 5th edition, 2010.

[45] M. M. Yee, Y. He, A. Soumyanarayanan, D.-J. Kim, Z. Fisk, and J. E. Hoffman. arXiv:1308.1085v2, 2013.

[46] C. E. Matt, H. Pirie, A. Soumyanarayanan, Y. He, M. M. Yee, P. Chen, Y. Liu, D. T. Larson, W. S. Paz, J. J. Palacios, M. H. Hamidian, and J. E. Hoffman. Phys. Rev. B, 101:085142, 2020 .

[47] C. E. Matt, H. Pirie, A. Soumyanarayanan, M. M. Yee, Y. He, D. T. Larson, W. S. Paz, J. J. Palacios, M. H. Hamidian, and J. E. Hoffman. arXiv:1810.13442v1, 2018.

[48] T. M. Mattox and J. J. Urban. Materials, 11(12):2473, 2018.

[49] C. M. McCarthy, C. W. Tompson, R. J. Graves, H. W. White, Z. Fisk, and H. R. Ott. Solid State Commun., 36(10):861 - 868, 1980.

[50] K. A. R. Mitchell. Contemp. Phys., 14(3):251-271, 1973.

[51] K. Momma and F. Izumi. J. Appl. Crystallogr., 44(6):1272-1276, 2011.

[52] F. D. Natterer, K. Yang, W. Paul, P. Willke, T. Choi, T. Greber, A. J. Heinrich, and C. P. Lutz. Nature, 543(7644):226-228, 2017.

[53] M. Neupane, S.-Y. Xu, N. Alidoust, G. Bian, D. J. Kim, C. Liu, I. Belopolski, T.-R. Chang, H.-T. Jeng, T. Durakiewicz, H. Lin, A. Bansil, Z. Fisk, and M. Z. Hasan. Phys. Rev. Lett., 114:016403, 2015.

[54] R. Nishitani, M. Aono, T. Tanaka, C. Oshima, S. Kawai, H. Iwasaki, and S. Nakamura. Surf. Sci., 93(2):535 - 549, 1980.

[55] C. Oshima, E. Bannai, T. Tanaka, and S. Kawai. J. Appl. Phys., 48(9):3925-3927, 1977.

[56] S. Otani, T. Aizawa, and Y. Yajima. J. Cryst. Growth, 234(2):431 - 434, 2002. 
[57] S. Otani, H. Nakagawa, Y. Nishi, and N. Kieda. J. Solid State Chem., 154(1):238 - 241, 2000 .

[58] J. S. Ozcomert and M. Trenary. Surf. Sci., 265(1):L227 - L232, 1992.

[59] J. S. Ozcomert and M. Trenary. J. Vac. Sci. Technol., A, 10(4):2581-2584, 1992.

[60] S. Patil, G. Adhikary, G. Balakrishnan, and K. Maiti. Appl. Phys. Lett., 96:092106-092106, 2010.

[61] S. Patil, G. Adhikary, G. Balakrishnan, and K. Maiti. J. Phys. Condens. Matter, 23(49):495601, 2011.

[62] J. P. Perdew, K. Burke, and M. Ernzerhof. Phys. Rev. Lett., 77(18):3865-3868, 1996.

[63] W. A. Phelan, S. M. Koohpayeh, P. Cottingham, J. A. Tutmaher, J. C. Leiner, M. D. Lumsden, C. M. Lavelle, X. P. Wang, C. Hoffmann, M. A. Siegler, N. Haldolaarachchige, D. P. Young, and T. M. McQueen. Sci. Rep., 6:20860, 2016.

[64] H. Pirie, Y. Liu, A. Soumyanarayanan, P. Chen, Y. He, M. M. Yee, P. F. S. Rosa, J. D. Thompson, D.-J. Kim, Z. Fisk, X. Wang, J. Paglione, D. K. Morr, M. H. Hamidian, and J. E. Hoffman. Nat. Phys., 16(1):52-56, 2020.

[65] M. Pohlit, S. Rößler, Y. Ohno, H. Ohno, S. von Molnár, Z. Fisk, J. Müller, and S. Wirth. Phys. Rev. Lett., 120:257201, 2018.

[66] S. V. Ramankutty, N. de Jong, Y. K. Huang, B. Zwartsenberg, F. Massee, T. V. Bay, M. S. Golden, and E. Frantzeskakis. J. Electron. Spectrosc. Relat. Phenom., 208:43 - 50, 2016.

[67] S. Rößler, L. Jiao, D. J. Kim, S. Seiro, K. Rasim, F. Steglich, L. H. Tjeng, Z. Fisk, and S. Wirth. Philos. Mag., 96(31):3262-3273, 2016.

[68] S. Rößler, L. Jiao, S. Seiro, P. F. S. Rosa, Z. Fisk, U. K. Rößler, and S. Wirth. Phys. Rev. B, 101:235421, 2020.

[69] A. L. Robinson. Science, 234:821-823, 1986.

[70] M. A. Rosentreter. Rastertunnelmikroskopie auf der GaAs(110)-Oberfläche bei Temperaturen von 8 K - 300 K. PhD thesis, Georg-August-Universität Göttingen, 1997.

[71] W. Ruan, C. Ye, M. Guo, F. Chen, X. Chen, G.-M. Zhang, and Y. Wang. Phys. Rev. Lett., 112:136401, 2014.

[72] W. M. H. Sachtler, G. J. H. Dorgelo, and A. A. Holscher. Surf. Sci., 5(2):221 - 229, 1966.

[73] E. Sani, L. Mercatelli, M. Meucci, L. Zoli, and D. Sciti. Sci. Rep., 7(1):718, 2017.

[74] K. M. Schmidt, O. Jaime, J. T. Cahill, D. Edwards, S. T. Misture, O. A. Graeve, and V. R. Vasquez. Acta Mater., 144:187 - 201, 2018. 
[75] K. M. Schmidt, S. T. Misture, O. A. Graeve, and V. R. Vasquez. ACS Omega, 4(1):65-72, 2019.

[76] M. Sera, S. Kobayashi, M. Hiroi, N. Kobayashi, and S. Kunii. Phys. Rev. B, 54:R5207R5210, 1996.

[77] Q. Si and F. Steglich. Science, 329(5996):1161-1166, 2010.

[78] N. Singh, S. M. Saini, T. Nautiyal, and S. Auluck. J. Phys. Condens. Matter, 19(34):346226, 2007.

[79] D. B. Sirdeshmukh. Curr. Sci., 72(9):631-640, 1997.

[80] N. N. Sirota, V. V. Novikov, V. A. Vinokurov, and Yu. B. Paderno. Phys. Solid State, 40(11):1856-1858, 1998.

[81] M. Sotoudeh, S. Rajpurohit, P. Blöchl, D. Mierwaldt, J. Norpoth, V. Roddatis, S. Mildner, B. Kressdorf, B. Ifland, and C. Jooss. Phys. Rev. B, 95(23):235150, 2017.

[82] S. Souma, H. Kumigashira, T. Ito, T. Takahashi, and S. Kunii. Physica B, 312-313:329 330, 2002.

[83] M. v. Stackelberg and F. Neumann. Z. Phys. Chem., 19B(1):314-320, 1932.

[84] F. Steglich, C. Geibel, K. Gloos, G. Olesch, C. Schank, C. Wassilew, A. Loidl, A. Krimmel, and G. R. Stewart. J. Low Temp. Phys., 95(1-2):3-22, 1994.

[85] P. Sun and F. Steglich. Phys. Rev. Lett., 110:216408, 2013.

[86] Z. Sun, A. Maldonado, W. S. Paz, D. S. Inosov, A. P. Schnyder, J. J. Palacios, N. Yu. Shitsevalova, V. B. Filipov, and P. Wahl. Phys. Rev. B, 97:235107, 2018.

[87] R. Svensson and L. Holmlid. Surf. Sci., 269-270:695 - 699, 1992.

[88] L. W. Swanson and T. Dickinson. Appl. Phys. Lett., 28(10):578-580, 1976.

[89] L. W. Swanson and D. R. McNeely. Surf. Sci., 83(1):11 - 28, 1979.

[90] A. Takase, K. Kojima, T. Komatsubara, and T. Kasuya. Solid State Commun., 36(5):461 464, 1980.

[91] H. Takeda, H. Kuno, and K. Adachi. J. Am. Ceram. Soc., 91(9):2897-2902, 2008.

[92] T. Takimoto. J. Phys. Soc. Jpn., 80(12):123710, 2011.

[93] A. Targowski. Comp. Civiliz. Rev., 51(51):7, 2004.

[94] J. Tersoff and D. R. Hamann. Phys. Rev. Lett., 50(25):1998-2001, 1983.

[95] J. Tersoff and D. R. Hamann. Phys. Rev. B, 31(2):805-813, 1985.

[96] M. Trenary. Sci. Technol. Adv. Mater., 13(2):023002, 2012. 
[97] M. A. Uijttewaal, G. A. de Wijs, and R. A. de Groot. J. Phys. Chem. B, 110(37):1845918465, 2006.

[98] G. Uimin. Phys. Rev. B, 55:8267-8279, 1997.

[99] S. Vogelgesang, G. Storeck, J. G. Horstmann, T. Diekmann, M. Sivis, S. Schramm, K. Rossnagel, S. Schäfer, and C. Ropers. Nat. Phys., 14:184, 2018.

[100] J. Voigt. Scanning tunneling microscopy on the LaB6 (001)-surface at low temperatures. Master thesis, Georg-August-Universität Göttingen, 2018.

[101] B. Voigtländer. Scanning Probe Microscopy: Atomic Force Microscopy and Scanning Tunneling Microscopy. Springer, Berlin Heidelberg, 2015.

[102] W. Wei, B. Lihong, L. Yingjie, C. Luomeng, and O. Tegus. J. Cryst. Growth, 415:123 126, 2015.

[103] S. Wirth, S. Rößler, L. Jiao, M. V. Ale Crivillero, P. F. S. Rosa, and Z. Fisk. Phys. Status Solidi B, page 2000022, 2020.

[104] M. Zhang, X. Wang, X. Zhang, P. Wang, S. Xiong, L. Shi, and Y. Qian. J. Solid State Chem., 182(11):3098 - 3104, 2009.

[105] W. Zhao, B. Xia, L. Lin, X. Xiao, P. Liu, X. Lin, H. Peng, Y. Zhu, R. Yu, P. Lei, J. Wang, L. Zhang, Y. Xu, M. Zhao, L. Peng, Q. Li, W. Duan, Z. Liu, S. Fan, and K. Jiang. Sci. Adv., 3(9):e1603231, 2017. 


\section{Publications, communications and supervised theses}

\section{Publications}

- P. Buchsteiner, F. Sohn, J. G. Horstmann, J. Voigt, M. Ciomaga Hatnean, G. Balakrishnan, C. Ropers, P. E. Blöchl, and M. Wenderoth Surface resonance of the $(2 \times 1)$ reconstructed lanthanum hexaboride $(001)$ cleavage plane: A combined STM and DFT study

Phys. Rev. B 100, 205407 (2019), DOI: 10.1103/PhysRevB.100.205407

- P. Buchsteiner, L. Harmsen, M. Ciomaga Hatnean, G. Balakrishnan, and M. Wenderoth

Surface analysis of the $\operatorname{PrB}_{6}$ (001) cleavage plane by scanning tunneling microscopy and spectroscopy

Phys. Rev. B 102, 205403 (2020), DOI: 10.1103/PhysRevB.102.205403

\section{Scientific communication}

- Philipp Buchsteiner, Geetha Balakrishnan, David P. Young, and Martin Wenderoth

Hexaboride preparation techniques for Scanning Tunneling Microscopy studies

DPG Spring Meeting, Regensburg, March 2016, poster presentation

- Philipp Buchsteiner, Geetha Balakrishnan, and Martin Wenderoth 
New insights into the LaB6 (001)-surface obtained by Scanning Tunneling Microscopy

DPG Spring Meeting, Dresden, March 2017, poster presentation

- Philipp Buchsteiner, Jan Voigt, Geetha Balakrishnan, and Martin Wenderoth The $2 x 1$ reconstruction of the LaB6 (001)-surface observed by Scanning Tunneling Microscopy

DPG Spring Meeting, Berlin, March 2018, poster presentation

- P. Buchsteiner, F. Sohn, J. Voigt, G. Balakrishnan, P. E. Blöchl, and M. Wenderoth

Resolving the electronic surface structure of Lanthanum Hexaboride (001)

DPG Spring Meeting, Regensburg, April 2019, poster presentation

\section{Supervised theses}

- Lisa Harmsen, Characterization of the (100) cleavage planes of LaB ${ }_{6}$ and $\mathrm{CeB}_{6}$, Bachelor thesis, Georg-August-Universität Göttingen (2017)

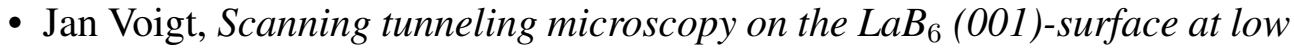
temperatures, Master thesis, Georg-August-Universität Göttingen (2018)

- Lisa Harmsen, Scanning tunneling microscopy on the $\operatorname{PrB}_{6}$ (001)-surface, Master thesis, Georg-August-Universität Göttingen (2019) 


\section{Danksagung}

$\mathrm{Zu}$ guter Letzt möchte ich mich bei all denjenigen bedanken, die auf die eine oder andere Weise dazu beigetragen haben, dass diese Arbeit erfolgreich war.

Natürlich ist an erster Stelle Martin Wenderoth zu nennen, bei dem ich meinen Bachelor, Master und dann auch die Promotion anfertigen durfte und mich somit meine gesamte akademische Laufbahn begleitet hat.

Mein Betreuungsausschuss bildete neben Martin auch Stefan Mathias und Peter Blöchl. Ich möchte mich bei Stefan für sein großes Interesse an meinem Projekt bedanken und für die Möglichkeit bei ihm eigene Photoemissionsexperimente durchzuführen. Außerdem bedanke ich mich für die Übernahme der Zweitkorrektur. Peter hat in zahlreichen Diskussionen mit seinem schier unerschöpflichen Wissen zur DFT mein Verständnis der Physik nachhaltig bereichert. Den Erfolg unserer Zusammenarbeit zeigt die gemeinsame Publikation.

Experimentalphysik, wie der Name vermuten lässt, lebt von Experimenten. Daher möchte ich Bernhard Spicher für seinen unermüdlichen Einsatz bei den vielen Reparaturen, Instandhaltungen und Erweiterungen der UHV-Technik danken. Insgesamt zeichnet sich die Gruppe Wenderoth durch eine hohe Hilfsbereitschaft füreinander aus. Das hat mir das Leben im Labor oft erleichtert. Auch möchte ich mich bei Jan Gerrit Horstmann bedanken, da er für mich die Messungen am ULEED durchgeführt hat. Ein wichtiger Schritt für das Gelingen dieser Arbeit war zudem die Kristallorientierung. Diese zeitaufwändigen Messungen wurden von Patrick Kirscht und Fabian Ziegler in der Gruppe von Götz Eckold durchgeführt und ich möchte mich ausdrücklich für diese große Unterstützung bedanken.

Besonders hervorheben möchte ich meine Studierenden Lisa und Jan. Mit euch zusammenarbeiten zu dürfen war ein wahrer Segen. Vor allem freut mich, dass Lisa nicht nur ihren Bachelor, sondern auch im Anschluss ihren Master unter 
meiner Betreuung machen wollte. Ich denke gerne an die Zeit mit euch zurück!

In Göttingen habe ich mich all die Jahre sehr wohl gefühlt, was nicht zuletzt an meinen lieb gewonnen Freundschaften liegt, die ich in dieser Stadt gefunden habe. Selbstverständlich möchte ich hier meine Wohnzimmer, das Café Schroeder und die Diva Lounge, hervorheben, in denen ich sehr viel schöne Zeit verbringen durfte. Geprägt war die Zeit in Göttingen auch von den vielen grandiosen Auftritten mit unserer Band, den knallhart gebutterten Lachsen. Für den sportlichen Ausgleich bei Wind und Wetter danke ich den Jungs von Calisthenics Göttingen. Bedanken möchte ich mich auch bei den Kutschern für die vielen schönen Abenteuer die ich mit euch erleben durfte.

Zur Korrektur von groben inhaltlichen Schwächen und Rechtschreibfehlern hat sich Thomas Dziuba bereit erklärt, danke dafür!

Meiner Familie, insbesondere meiner Mutter, meinem Vater und meinem Bruder, möchte ich an dieser Stelle meinen Dank aussprechen. Eure Unterstützung hat all die Jahre sehr gut getan. 


\section{Additional notes}

The initially submitted Dissertation differs from the present version. The initial Section 8.1 consisted of a submitted manuscript version, which was, at that point, under current review. Shortly after the thesis submission the manuscript was accepted for publication with minor adaptions. In consequence, the present version contains the accepted manuscipt version and the respective DOIs were added to both, the $\mathrm{LaB}_{6}$ publication, see Chapter 7 , and the $\operatorname{PrB}_{6}$ publication, see Chapter 8 . Furthermore, the DOIs and citations were added to Publications, communications and supervised theses Chapter as well. Please find a brief summary of the major changes of Section 8.1 below.

The former Fig. 8.1 was extended by an additional large scale topography of the chainlike $(2 \times 1)$ reconstruction and additional cross sections. In Fig. 8.2 the spectroscopies in (g), (h) and (l) were modified. After the above mentioned change, the bias voltage ranges in (h) and (l) are equal to enhance the comparability between these two spectra. Furthermore, additional template averages were included for the Fig. 8.3 and Fig. 8.4 in the appendix as new figures. Figure 8.10 was also included as new figure. Since the above mentioned figures contain template averages, to the new Methods part an explanation was added of how these templates were calculated. The nomenclature of the uniform Pr termination was changed to stripelike reconstruction in both Section 8.1 and Chapter 9. Also, the nomenclature of $4 f$ states was changed to $4 f$-related states in Section 8.1.

Furthermore, to the caption of Fig. 2.1 a note was added, that the schematic drawing were created with VESTA [51]. 